
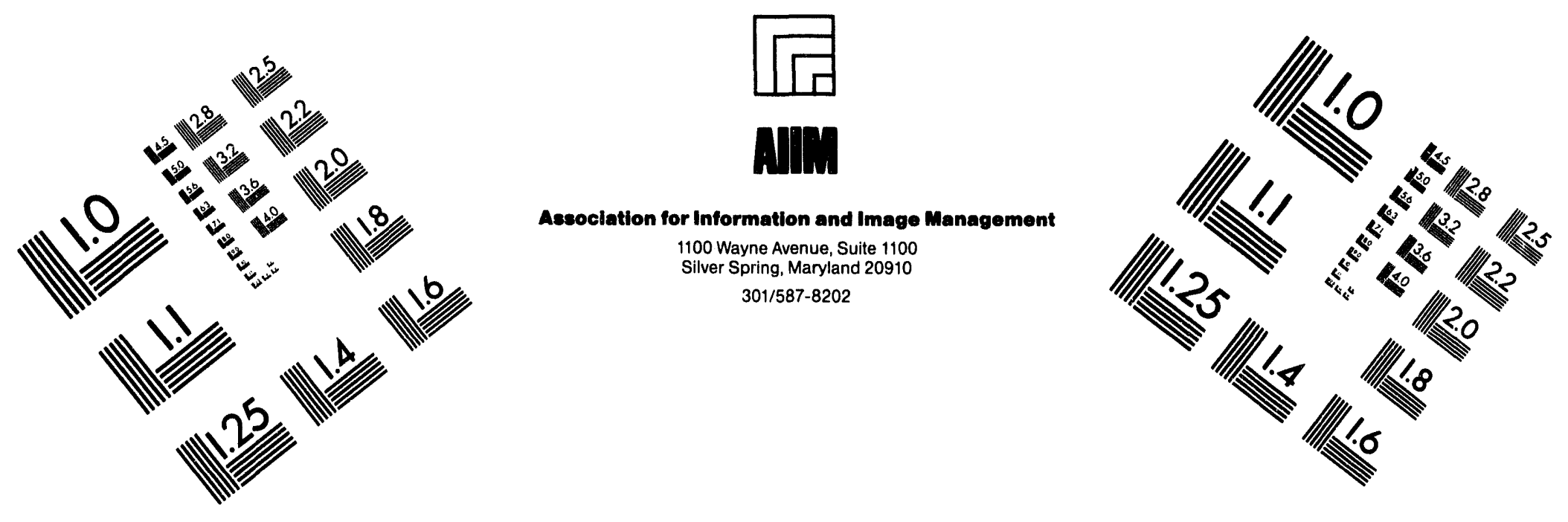

\title{
Centimeter
}

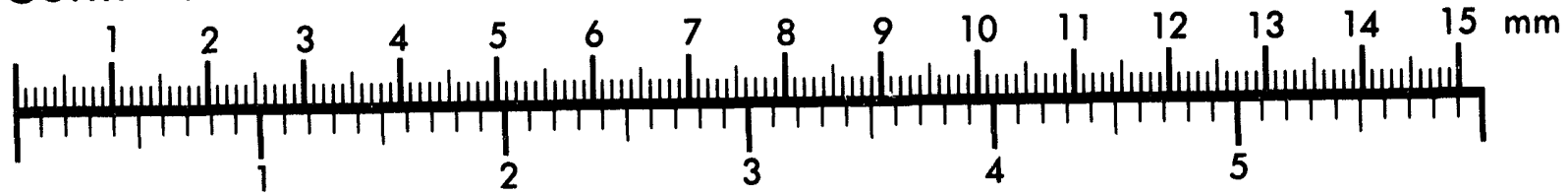
Inches
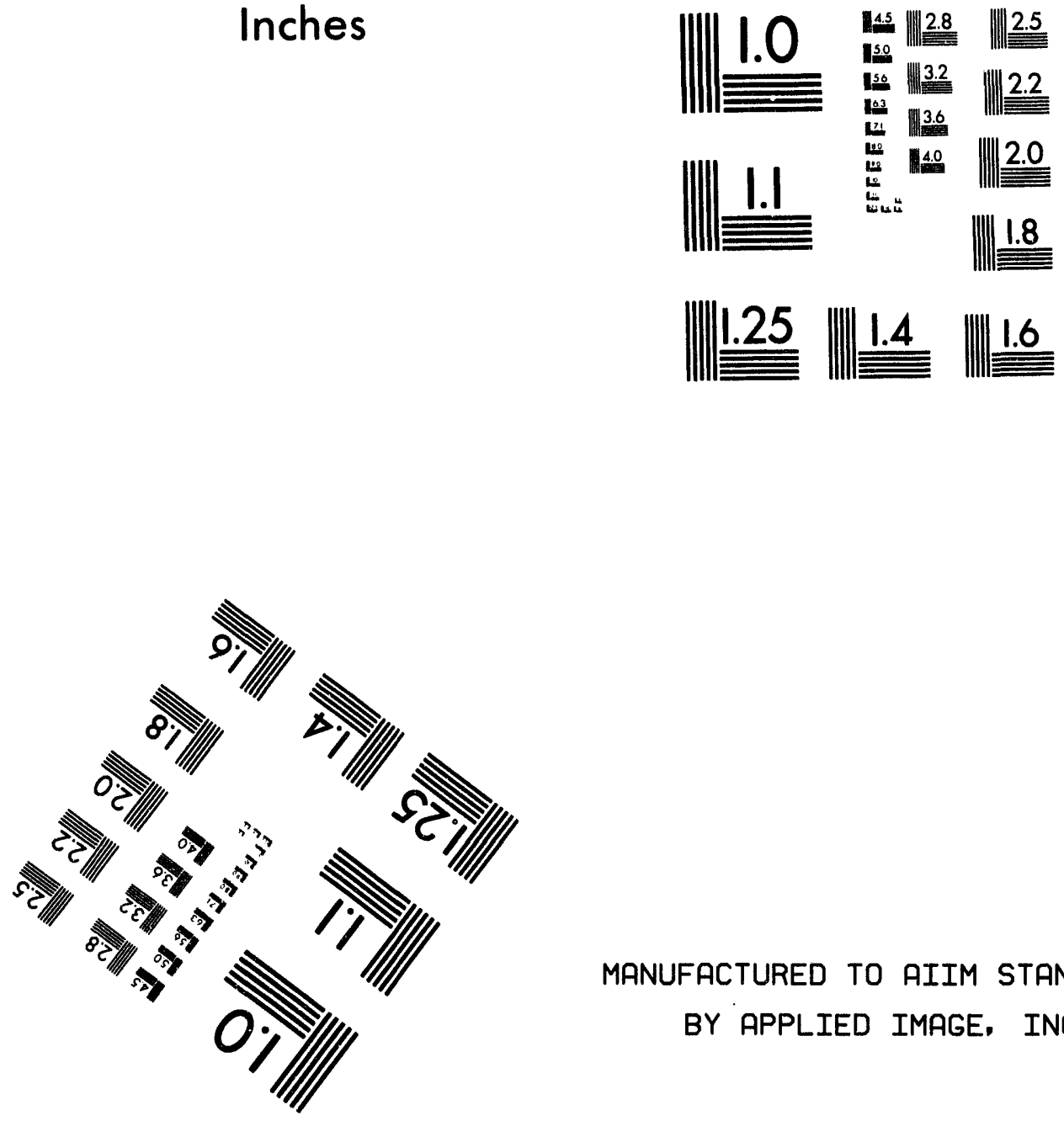

MANUFACTURED TO AIIM STANDARDS

BY APPLIED IMAGE, INC.

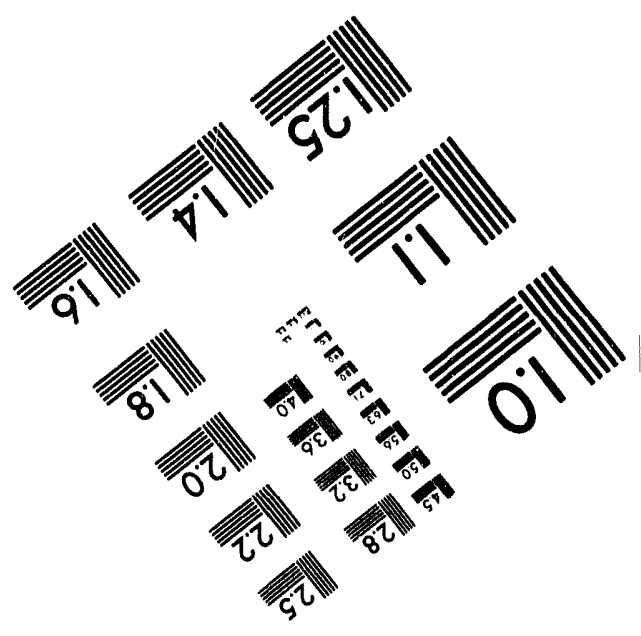


Cost Estimates for Near-term Deployment of Advanced Trafic Management Systems

Final Report

by

S. S. Stevens

Center for Transportation Analysis

Oak Ridge National Laboratory

and

S. M. Chin

Center for Transportation Analysis

Oak Ridge National Laboratory

February 15, 1993

Prepared for the

Federal Highway Administration

Prepared by the

Center for Transportation Analysis

Oak Ridge National Laboratory

P. O. Box 2008

Oak Ridge, TN 37831, U. S. A

managed by

MARTIN MARIETTA ENERGY SYSTEMS, INC.

for the

UNITED STATES DEPARTMENT OF ENERGY

under contract no. DE-AC05-84OR21400 
FINAL REPORT

Cost Estimates for Near-term Deployment

of Advanced Traffic Management Systems

\section{S. S. Stevens}

S. M. Chin

February 15, 1993

\section{Objective}

The objective of this study is to provide cost estimates for the next five years for engineering, design, installation, operation and maintenance of Advanced Traffic Management Systems (ATMS) in the largest 75 metropolitan areas in the United States. The areas are listed in order of total population in Table 1.

\section{Scope of Report}

This report gives estimates for deployment costs for ATMS in the next five years, subject to the qualifications and caveats set out in following paragraphs. The report considers infrastructure components required to realize fully a functional ATMS over each of two highway networks (as discussed in the Section describing our general assumptions) under each of the four architectures identified in the MITRE Intelligent Vehicle Highway Systems (IVHS) Architecture studies [Ref 1]. The architectures are summarized in this report in Table 2. Estimates are given for eight combinations of highway networks and architectures.

\section{Summary of Results}

We estimate that it will cost between $\$ 8.5$ Billion (minimal network) and $\$ 26$ Billion (augmented network) to proceed immediately with deployment of ATMS in the largest 75 metropolitan areas. Costs are given in 1992 dollars, and are not adjusted for future inflation. Our estimates are based partially on completed project costs, which have been adjusted to 1992 dollars. We assume that a particular architecture will be chosen; projected costs are broken out by architecture. Costs are divided among areas by population as shown in Tables 3-4. Total cost per year is shown in Tables 5-6. 


\section{Approach}

For this report, we have requested information about the component costs of ATMS or IVHS projects from a number of sources (see References and Sources) representing various sizes of metropolitan areas. The numbers from different sources were compared, and unit costs were estimated. Using available data for metropolitan area populations and highway mileages, total cost projections were computed for each of the 75 largest metropolitan areas. The estimated area costs were summed within each size category and across categories to give the overall cost estimate. The calculations were repeated for various assumptions about highway networks and IVHS architectures.

For this report, we have collected data from a number of areas. Those data allow us to adjust our estimates to account for geographical differences in costs, and to take into account the current state of operation, construction, or planning within some of the specific areas considered. We have projected costs by summing what we do know and extrapolating cost numbers for areas for which we do not have complete data.

We also base our estimates on the following assumptions:

\section{General Assumptions}

- The architectural options are those identified in the MITRE Corp. Working Paper 91-W-00512, Alternative IVHS Architectures as Strawman Architectures 1-4. The options are summarized in Table 2.

- Only infrastructure components are included. In-vehicle components are specifically excluded.

- For this report, highway centerline mileages (both directions of travel) for the metropolitan areas were derived from the Federal Highway Administration's (FHWA's) Highway Performance Monitoring System. We have considered two highway networks for our estimates. The lowest-cost configuration considers freeways and only the set of arterials that could be major diversion routes within a metropolitan area, which we call the minimal network. For the second network, we include also many of the remaining arterials within the metropolitan area, under the supposition that traffic would be monitored and signals controlled within the entire freeway and arterial network by the 
ATMS. The second network is referred to as the augmented network, but it still excludes signalized intersections on less-important streets within an area. If our other assumptions are accurate, calculations based on the minimal network and on the augmented network may represent practical lower and upper bounds on the required funds. Projects now in active planning stages for which we obtained data usually consider networks no larger than our minimal networks. We exercised some engineering judgement in assigning numbers of miles of arterials to metropolitan areas, since details of reasonable ATMS project scope will be specific to each area. Further discussion of . highway mileages is included in the final section of this report.

- We assume interchange spacing on freeways to be two miles.

- Reviewers of a draft of this report suggested a five-mile spacing for variablemessage signs. We have used that number, even though one active ATMS design has somewhat more dense spacing. If signs were paired, for example, with one sign for each direction of travel near a critical interchange, the five-mile average spacing would be consistent with ten-mile critical-interchange spacing.

- As discussed in the MITRE paper, each architecture includes traffic sensors along freeways and arterials, a communication network linking the sensors to a traffic management center, variable message signs (and, in two architectures, beacons) at strategic locations along the highway network, traffic control devices at ramps and intersections (assumed to be coordinated within an area), and a computer system adequate to analyze the collected data and disseminate the required guidance and control information. Three of the four strawman architectures include one- or two-way radio communication between vehicles and the infrastructure. Some features of the architectures are given in the MITRE paper.

- For this report, traffic sensors are assumed to be inductive loops, the communication trunks are assumed to be fiber optic cables, and the computer facility is assumed to be a network made up of front-end data acquisition computers, one or more central computation/database servers, and workstations for operator interaction.

- Software cost is based on estimates from firms that now supply such software. No allowance is made for either Federal government development of generic software or a significant third-party software development effort, although the cost of software considerably exceeds the cost of hardware. 
- Deployment is assumed to "ramp up." That is, larger projects will begin by next year, with many smaller projects phasing in over the five-year period. The projects will have multi-year duration, with larger projects of course taking somewhat longer than small ones, though not proportionally longer, since presumably more intensive effort will be expended on the larger ones. Our estimates assume that efforts will be well under way in all 75 metropolitan areas by the end of the five-year period.

\section{Assumed Component Costs}

Planning, engineering, and design are assumed to be required in proportion to the expected total project size. Our assumed costs are based partly on the engineering judgement of our consultants. For this interim report we assume planning is 5\%-10\% complete for large metropolitan areas, and not significantly begun for mid-sized and smaller areas.

Sensor hardware is assumed to be inductive loops with moderately intelligent controller/interfaces, installed at 0.3 -mile intervals in each direction of travel for freeways and at intersections for arterials. Installeo cost per mile is assumed to be $\$ 50 \mathrm{~K}$, a figure between those usually quoted for southern and northern regions of the country. (We believe that sensor technology other than inductive loops might give a significant improvement in benefit/cost performance within the period under consideration. If development of image-processing hardware and algorithms solves the remaining ambientlight problems, it will be possible to use video for the more mundane traffic-counting functions, with low-bandwidth count data reporting requirements. The same cameras will then be widely available for high-bandwidth incident detection/verification, a concept that one consultant calls "video-on-demand." Both cameras and image-processing hardware are participants in the on-going cost reduction process for solid state equipment, and the installed cost of a camera and controller may well be less than the installed cost of an inductive loop and controller within the time frame of ATMS deployment.)

Variable-message signs (VMS) are used or proposed in several projects for which we have some cost information. We assume that variable message signs will be spaced about every five miles on freeways, and extrapolate our sign-cost data to give an installed cost of $\$ 40 \mathrm{~K} /$ mile. (In urban areas with well-developed highway networks, where opportunities for alternate routes occur more often than every five miles, it is possible that VMS spacing will be less than five miles.) Ramp metering signals are assumed to be used extensively, at a cost of $\$ 30 \mathrm{~K} /$ mile. Replacement cost for obsolete intersection 
signals is likely to be significant. We have guessed that half the traffic signals now in use would need to be replaced or upgraded. Costs are included below for extensive cabling to connect the signals into an ATMS.

Beacons for two-way communications between the infrastructure and vehicles are features of Architectures 2 and 4. Installed cost projections for beacon hardware are somewhat speculative. (We considered costs from a proposed project that uses beacons, but asked our consultants to evaluate our preliminary estimates.) We assume beacons at 0.5 mile intervals along freeways and principal arterials, and guess the cost to be $\$ 50 \mathrm{~K} / \mathrm{mile}$. Note that using beacons presumes high-speed communication or very clever coding of information, and that beacon cost is one differentiating feature among the MITRE architectures.

Three architectures assume radio communication between vehicles and the infrastructure. The infrastructure components required are estimated to cost $\$ 15 \mathrm{~K} / \mathrm{mile}$ (Architectures 1, 2, and 3), with associated installation and maintenance costs. Radio broadcasting can cover an area, as opposed to being a linear process aligned with a roadway. That reduces the number of transmitters required to cover a highway network. However, there are several potential radio technologies that could be utilized, and the MITRE architecture discussions leave great freedom to choose a particular broadcast technique, with its associated costs. Accordingly, our numbers here are not very conclusive; we expect that further architecture studies will enable better cost estimates for this function. In any case, we doubt that radio-broadcast equipment will be a cost driver in any ATMS architecture that is ultimately chosen for implementation.

Sensors and signals for an ATMS are assumed to be linked into a network by a communication trunk line with interfaces and local links at individual locations. We assume that very little of the required communication network is now in place. We assume fiber optic cable in conduit, because fiber optic cable offers orders of magnitude more capability for transmitting information than twisted-pair cable. Recent experience and several studies indicate that fiber optics is becoming available at little extra cost, and use of fiber optics will provide enough bandwidth to effectively "future-proof" the communication network for the foreseeable future. Several reputable experts suggested that we consider geographical differences in the cost of installing conduit, which dominates the communication trunk cost. Installed cost per mile for cable is assumed to be $\$ 125 \mathrm{~K}-\$ 175 \mathrm{~K}$; both figures are within the range quoted for different climates and areas. As one can see from the graph on page 5 of the estimation model in the 
appendix, the cost of providing such a conduit/cable communication network dominates the overall system installation costs. Since data collection is common to all proposed ATMS architectures, the cost dominance of the communications (actually, the cost of installing conduit) tends to minimize variations in our cost estimates for different architectures. We assume a distributed traffic management architecture, which is usually assumed to require lower overall communication cost than a strongly centralized system.

The cost of computer hardware cannot be projected reliably from costs on record for projects now in successful operation, because of the remarkably rapid increase in computational power per dollar, for which the computer industry is justly famous. We consider instead, the computational power and user interfaces required for recent ORNL projects, such as the AMC Deployment Analysis System project for the Airlift Mobility Command, which perform somewhat similar (and similarly difficult) functions. We assume a modular, fault-tolerant client-server architecture, where a module consists of a network of small data-acquisition/concentration computers, perhaps some multiplexer or concentrator computers, one or a few computation or database server(s), and three to five client workstations for operators. The data-handling computers for a such a modular Traffic Management Center (TMC) and the operator workstations are assumed to be linked in a high-availability local area network. Modules within a large metropolitan area are assumed to be linked by a wide area network. Projected cost is somewhat sensitive to whether the route-planning function is carried out in the vehicle or in the TMC computers, but installed cost of such a system is unlikely to be significantly more than \$175K per module. Other (e.g., mini-computer-based) computer hardware could be selected if different criteria such as backward compatibility with existing software were a dominant consideration or if a strongly centralized system were chosen, but it is unlikely that ATMS architectures now under consideration will need more expensive hardware to realize full functionality.

Recent experience suggests that computer equipment often is obsolete before it fails, and that it has an effective lifetime of no more than five years. Computer equipment upgrades can be considered to be an annual "maintenance" cost of about $\$ 30 \mathrm{~K}$ per module per year.

One module should serve a small metropolitan (or equivalent) area, but the actual number and location of TMCs in a metropolitan area are likely to be influenced by the political boundaries within the area. However, because we have no reasonable basis for second-guessing politicians, we have assumed that TMCs will be distributed by traffic 
requirements related to population, and that a TMC will serve about half a million people. By these criteria, at least one TMC will be required in each area, and the requirements will scale up to more than a dozen distributed throughout an area such as the greater New York area.

The development of unique ATMS software for each metropolitan area does not seem to us to be justified by differences in requirements. We therefore assume that the one-time cost for design and development of the basic software is distributed over at least several sites. The resulting cost per module, $\$ 300 \mathrm{~K}$, is based on estimates provided by two vendors of such software. Basic software cost should not be very specific to size of the area, but the cost of creating the site-specific highway network data base is; we include a per-mile cost for "instantiation" of the software. There is also a recurring cost of $\$ 15 \mathrm{~K}$ per year for software maintenance and upgrading.

Our consultants suggest that regional traffic management centers - the modules discussed above - could occupy about 1000 square feet of building space, perhaps with a larger central coordinating site in the largest metropolitan areas. (This space requirement contains a tacit assumption that traffic management within larger metropolitan areas will be distributed among the local districts within the area. If the local governments within a large area choose to consolidate all management functicris in a single central traffic management center, a decision strongly influenced by the chosen IVHS architecture, several cost categories would be influenced. Our consultants suggest that most, if not all, of their new systems are likely to be modular and distributed. Current practice and trends in other computer-based business and control domains suggest a strong and increasing tendency toward distributing functionality.) Projects for which we received cost data tended to spend millions of dollars constructing new buildings. We do not believe that large central facilities are technically justified, and have assumed a distributed system, with an initial cost per local TMC of $\$ 200 \mathrm{~K}$, with a continuing yearly cost of $\$ 20 \mathrm{~K}$. These figures might represent the cost of adapting existing office space which is thereafter leased.

Successful deployment of advanced traffic management systems will require the creation of a cadre of well-trained professionals who are familiar with and proficient at traffic management, and are also comfortable with and capable of using and maintaining the computer-based advanced technology of the future Traffic Management Center. After visiting several existing showcase TMCs, we estimated that - ideally - each TMC module will require three operators per rush hour shift, one hardware maintenance 
expert, and one software maintenance expert. That staffing level translates roughly into salary cost of $\$ 750 \mathrm{~K}$ per year. However, our consultants indicate that they believe that few metropolitan areas are really likely to staff at that level. The estimates of recurring cost in this report are accordingly based on our experts' suggestion of about $\$ 400 \mathrm{~K}$ per year per local TMC. (Using the larger figure -- with three operators instead of two, and an additional support staff member - increases operating costs by about 5 per cent.) Large, multi-shift TMC operations can realize economies of scale by using fewer maintenance personnel and very small operations might combine the hardware and software maintenance functions into a single position or share maintenance staff with other municipal functions, but every physically distinct site will require some maintenance staffing.

\section{Discussion of Computational Procedures and Methods}

\section{Computational Procedures}

Summary costs were computed from the component data by the procedures shown in the attached MathCADm model. Individual metropolitan area costs were estimated for each highway network configuration paired with each architectural option, considering all of the available data about status of current plans, geographically significant cost variations, and unique metropolitan area needs. The resulting individual estimates were then summed for overall projected costs.

The time distribution of costs was projected by assuming a forward-skewed timecost distribution for each project (an example is plotted in the Appendix), assuming that larger projects take longer, and that project start dates will be distributed over the next two years (details are in the MathCAD model). The calculated distributions of individual project costs were then summed to give an overall distribution of costs.

\section{Precision, Accuracy, and Range of Applicability}

It became apparent to us almost as soon as we began to collect cost data that there was great variability in cost reports and cost estimates for similar work. We believe - as do our consultants - that the variability results both from actual variation in costs, and from great variability in reporting/estimating costs (for whatever technical or political realities). Accordingly, we have made our projections based on "reasonable compromise" unit costs that fall within the reported and estimated numbers. Hence our 
projections are "reasonable" extrapolations, and should not be compared directly to the actual costs or estimates for any specific project. Component cost assumptions are listed in Table 7.

In addition, we realize that there are many combinations of components that could be implemented as ATMS and that costs for the components will vary according to forces in the market place. We have therefore built a fairly general model that can be used to project costs for any chosen set of components, as long as unit data are available or can be estimated, and as long as some assumptions can be made about the placement of elements on the highway network.

For each element of cost, the reported or estimated unit cost typically varied by at least a factor of two. We therefore have not made any corrections to our projections to account for "small" effects like inflation.

\section{Opportunities for Further Efforts}

\section{Response to Definition of Architectures}

Our model has been constructed to allow revised project scope and unit cost data to be entered when such data become available. In particular, as IVHS architectures become more fully defined, it will become easier to differentiate between suggested configurations and the essential components of each architecture will be known. As unit costs for those components are determined, it will be possible to better estimate the cost of implementation of a particular configuration in a specific situation. Such an estimate, of course, is no substitute for a complete engineering study, but our model could provide useable rough estimates as the scope of potential projects is considered.

\section{$\underline{\text { Refinement of Mileage Estimates }}$}

Metropolitan statistical area classification is a statistical standard, developed for use by Federal agencies in the production, analysis, and publication of data on metropolitan areas. Three basic types of metropolitan areas are recognized by the Office of Management and Budget (OMB) for Federal Statistical purposes: metropolitan statistical areas (MSAs), consolidated metropolitan statistical areas. (CMSAs), and primary metropolitan statistical areas (PMSAs). 
A metropolitan statistical area is a geographic area consisting of a large population nucleus (i.e., census-defined urban area) along with adjacent communities that have a high degree of economic and social integration with the nucleus. Each MSA contains one or more central counties which contain the area's main population concentration. An MSA may also include outlying counties that have close social and economic interaction with the central county. However, such outlying counties must have a specified population density and level of commuting with the central counties. If an area has more than one million residents and meets specified population and commuting criteria, PMSAs may be identified. PMSAs consist of a large urbanized county or cluster of counties that have strong internal economic and social links, in addition to close ties to neighboring areas. Local opinion must also support separate recognition of PMSAs. Unlike MSAs, PMSAs do not have to have a central city. When PMSAs are defined, the MSA of which they are components is redesignated as a CMSA.

The list of metropolitan areas to be included in this project was provided by the Federal Highway Administration. This list consists mostly of MSAs and CMSAs. Unfortunately, many of these regions contain large areas that are not populous enough to warrant the implementation of Advanced Traffic Management Systems. Thus, to estimate the cost of implementing ATMS for such sparsely populated areas seemed to us not to be within the scope of this project. To support this position, the following two examples are given.

The Montgomery MSA in Alabama includes Autauga, Elmore, and Montgomery counties. The City of Montgomery, located in Montgomery County, has more than 100,000 residents. However, neither Autauga nor Elmore county has a single city with a population of more than 25,000 . Thus, it seems unlikely that the highways, expressways, and arterials in these two counties would be included in ATMS projects.

The Los Angeles-Anaheim-Riverside CMSA includes the Anaheim-Santa Ana, Los Angles-Long Beach, Oxnard-Ventura, and Riverside-San Bernardino PMSAs. The Riverside-San Bernardino PMSA extends all the way to the Arizona and Nevada state boundaries. Although the cities of San Bernardino and Riverside have more than 100,000 residents, much of the PMSA has a small population, and a significant portion of this PMSA lies within the desert. Thus, it seems unlikely that ATMS of the nature considered in this study would be implemented in these areas. 
Therefore, in order to minimize the effects of including such large areas with relatively small populations, those urban areas having more than 100,000 residents as defined by individual states and reported in the HPMS were used to estimate the highway mileage for future IVHS projects.

The Highway Performance Monitoring System (HPMS), developed by the Federal Highway Administration, is a program management tool used to periodically assess the national highway system in terms of extent and physical condition; the safety, efficiency, and economy of the system in serving the movement of goods and people; and the impacts of existing national programs and policies. HPMS is also used to assess the potential impacts of proposed programs, policies, and alternatives. The system promotes the efficient use of decreasing financial resources and provides invaluable information to decision makers.

This study provides the FHWA with a preliminary estimate of the costs involved in implementing ATMS projects in urban areas. The estimates are based on average costs per mile and highway mileage estimates for plausible networks in the major metropolitan areas.

It seems desirable to have a system that would be capable of tracking summary cost estimates for the major metropolitan areas by allowing entry of data about the kinds of technologies to be used and the exact expressway and arterial sections as they are chosen for ATMS system design. We believe that a minimal geographically linked database could be developed to accurately track estimates of the cost of implementing ATMS and/or other IVHS-related systems. Such summary estimates of course would not be a substitute for preliminary engineering design, but they could be extremely useful both to administrators of a national program, and to local decision-makers who need to prepare budget estimates for the political review process. 
Table 1. Metropolitan Areas Ordered by Population

$\begin{array}{llllll}\text { New York } & 1 & \text { Sacramento } & 26 & \text { Honolulu } & 51 \\ \text { Los Angeles } & 2 & \text { Portland } & 27 & \text { Austin } & 52 \\ \text { Chicago } & 3 & \text { Norfolk } & 28 & \text { Las Vegas } & 53 \\ \text { San Francisco } & 4 & \text { Columbus OH } & 29 & \text { Raleigh-Durham } & 54 \\ \text { Philadelphia } & 5 & \text { San Antonio } & 30 & \text { Scranton } & 55 \\ \text { Detroit } & 6 & \text { Indianapolis } & 31 & \text { Tulsa } & 56 \\ \text { Boston } & 7 & \text { New Orleans } & 32 & \text { Grand Rapids } & 57 \\ \text { Dist of Columbia } & 8 & \text { Buffalo } & 33 & \text { Allentown } & 58 \\ \text { Dallas-FtWorth } & 9 & \text { Charlotte } & 34 & \text { Fresno } & 59 \\ \text { Houston } & 10 & \text { Providence } & 35 & \text { Tucson } & 60 \\ \text { Miami } & 11 & \text { Hartford } & 36 & \text { Syracuse } & 61 \\ \text { Atlanta } & 12 & \text { Orlando } & 37 & \text { Greenville SC } & 62 \\ \text { Cleveland } & 13 & \text { Salt Lake City } & 38 & \text { Omaha } & 63 \\ \text { Seattle } & 14 & \text { Rochester } & 39 & \text { Toledo } & 64 \\ \text { San Diego } & 15 & \text { Nashville } & 40 & \text { Knoxville } & 65 \\ \text { Minneapolis } & 16 & \text { Memphis } & 41 & \text { El Paso } & 66 \\ \text { St Louis } & 17 & \text { Oklahoma City } & 42 & \text { Harrisburg } & 67 \\ \text { Baltimore } & 18 & \text { Louisville } & 43 & \text { Bakersfield } & 68 \\ \text { Pittsburgh } & 19 & \text { Dayton } & 44 & \text { New Haven } & 69 \\ \text { Phoenix } & 20 & \text { Greensboro-WS } & 45 & \text { Springfield MA } & 70 \\ \text { Tampa } & 21 & \text { Birmingham } & 46 & \text { Baton Rouge } & 71 \\ \text { Denver } & 22 & \text { Jaclasonville } & 47 & \text { Little Rock } & 72 \\ \text { Cincinnati } & 23 & \text { Albany } & 48 & \text { Charleston } & 73 \\ \text { Milwaukee } & 24 & \text { Richmond } & 49 & \text { Youngstown } & 74 \\ \text { Kansas City } & 25 & \text { West Palm Beach } & 50 & \text { Wichita } & 75 \\ & & & & & \end{array}$


Table 2. Summary Comparison of MITRE Architectures

[Derived directly from Table 3.2 of MITRE Report]

Architecture 1:

Uncoupled route selection/traffic control

In-vehicle route selection

In-vehicle map database

Two-way wide-area radio communications

Architecture 2:

Partially coupled route selection/traffic control

In-vehicle route selection

In-vehicle map database

Two-way localized (beacon) communications

Architecture 3:

Fully coupled route selection/traffic control

Centralized route selection (with real-time data)

In-vehicle route selection (with static data) when real-time routing is unavailable

In-vehicle map database

Two-way cellular radio communications (digital technology)

One-way traffic information broadcast to vehicles

Architecture 4:

Uncoupled route selection/traffic control

Centralized route selection

No in-vehicle map database

Two-way localized (beacon) communications 
Table 3. Projected Total ATMS Cost by Archilecture and Metro Area Size (Minimal highway network)

\begin{tabular}{|c|c|c|c|c|}
\hline & Arch 1 & Arch 2 & Arch 3 & Arch 4 \\
\hline 9 Regions with population $>4 M$ & \$3.81B & $\$ 4.4 B$ & $\$ 3.82 \mathrm{~B}$ & $\$ 4.41 B$ \\
\hline O\&M per year & $\$ 276 M$ & $\$ 335 M$ & $\$ 277 \mathrm{M}$ & \$335M \\
\hline 12 Regions with $2 \mathrm{M}<$ pop $<4 \mathrm{M}$ & $\$ 1.91 B$ & $\$ 2.24 B$ & $\$ 1.91 B$ & $\$ 2.25 B$ \\
\hline O\&M per year & $\$ 143 M$ & $\$ 176 M$ & $\$ 144 M$ & \$177M \\
\hline 18 Regions with $1 \mathrm{M}<$ pop $<2 \mathrm{M}$ & $\$ 1.51 B$ & $\$ 1.75 B$ & $\$ 1.52 \mathrm{~B}$ & $\$ 1.76 \mathrm{~B}$ \\
\hline O\&M per year & $\$ 114 \mathrm{M}$ & $\$ 138 \mathrm{M}$ & \$115M & $\$ 139 M$ \\
\hline 36 Regions with pop $<1 M$ & $\$ 1.33 B$ & $\$ 1.56 \mathrm{~B}$ & $\$ 1.34 \mathrm{~B}$ & $\$ 1.56 \mathrm{~B}$ \\
\hline O\&M per year & $\$ 103 \mathrm{M}$ & $\$ 125 \mathrm{M}$ & $\$ 103 M$ & $\$ 125 M$ \\
\hline Total Installed Cost & $\$ 8.57 B$ & $\$ 9.95 B$ & $\$ 8.59$ & \$9.97B \\
\hline Total Annual O\&M & $\$ 640 \mathrm{M}$ & \$770M & $\$ 640 M$ & \$780M \\
\hline
\end{tabular}

Table 4. Projected Total ATMS Cost by Architecture and Metro Area Sixe (Augmented highway network)

\begin{tabular}{|c|c|c|c|c|}
\hline & Arch 1 & Arch 2 & Arch 3 & Arch 4 \\
\hline 9 Regions with population $>4 \mathrm{M}$ & $\$ 10.9 B$ & S11.5B & \$10.9B & \$11.5B \\
\hline O\&M per year & \$710M & $\$ 768 \mathrm{M}$ & $\$ 710 \mathrm{M}$ & $\$ 769 \mathrm{M}$ \\
\hline 12 Regions with $2 \mathrm{M}<$ pop $<4 \mathrm{M}$ & $\$ 5.45 B$ & $\$ 5.79 \mathrm{~B}$ & S5.46B & \$5.79B \\
\hline O\&M per year & $\$ 367 \mathrm{M}$ & \$400M & \$368M & $\$ 401 M$ \\
\hline 18 Regions with $1 \mathrm{M}<$ pop $<2 \mathrm{M}$ & $\$ 4.31 B$ & \$4.45B & $\$ 4.32 B$ & \$4.55B \\
\hline O\&M per year & $\$ 289 \mathrm{M}$ & $\$ 313 M$ & S289M & \$313M \\
\hline 36 Regions with pop $<1 \mathrm{M}$ & $\$ 3.8 B$ & $\$ 4.03 B$ & $\$ 3.81 \mathrm{~B}$ & $\$ 4.03 B$ \\
\hline O\&M per year & $\$ 258 \mathrm{M}$ & $\$ 281 \mathrm{M}$ & $\$ 259 \mathrm{M}$ & $\$ 281 \mathrm{M}$ \\
\hline Total Installed Cost & $\$ 24.46 B$ & $\$ 25.84 B$ & $\$ 24.48 B$ & $\$ 25.86 B$ \\
\hline Total Annual O\&M & $\$ 1.62 \mathrm{~B}$ & $\$ 1.76 B$ & $\$ 1.63 B$ & $\$ 1.76 B$ \\
\hline
\end{tabular}


Table 5. Projected Total ATMS Cost by Architecture and Year (Minimal highway network)

\begin{tabular}{|c|c|c|c|c|}
\hline & Arch 1 & $\operatorname{Arch} 2$ & Arch 3 & Arch 4 \\
\hline FY93 Capital & $\$ 0.97 \mathrm{~B}$ & $\$ 1.12 \mathrm{~B}$ & $\$ 0.97 \mathrm{~B}$ & $\$ 1.12 \mathrm{~B}$ \\
\hline FY94 Capital & $\$ 2.03 B$ & $\$ 2.35 \mathrm{~B}$ & $\$ 2.03 \mathrm{~B}$ & $\$ 2.36 \mathrm{~B}$ \\
\hline FY95 Capital & $\$ 2.65 B$ & $\$ 3.08 \mathrm{~B}$ & $\$ 2.66 \mathrm{~B}$ & $\$ 3.09 \mathrm{~B}$ \\
\hline FY96 Capital & $\$ 2.03 B$ & $\$ 2.36 \mathrm{~B}$ & $\$ 2.04 \mathrm{~B}$ & $\$ 2.37 \mathrm{~B}$ \\
\hline FY97 Capital & \$0.89B & $\$ 1.03 B$ & \$0.89B & $\$ 1.03 B$ \\
\hline Total Capital & $\$ 8.57 \mathrm{~B}$ & $\$ 9.95 B$ & $\$ 8.59 \mathrm{~B}$ & \$9.97B \\
\hline
\end{tabular}

Table 6. Projected Total ATMS Cost by Architecture and Year (Augmented highway network)

\begin{tabular}{lllll} 
& Arch 1 & Arch 2 & Arch 3 & Arch 4 \\
FY93 Capital & $\$ 2.78 \mathrm{~B}$ & $\$ 2.93 \mathrm{~B}$ & $\$ 2.78 \mathrm{~B}$ & $\$ 2.93 \mathrm{~B}$ \\
FY94 Capital & $\$ 5.79 \mathrm{~B}$ & $\$ 6.12 \mathrm{~B}$ & $\$ 5.79 \mathrm{~B}$ & $\$ 6.12 \mathrm{~B}$ \\
& & & & \\
FY95 Capital & $\$ 7.56 \mathrm{~B}$ & $\$ 8.0 \mathrm{~B}$ & $\$ 7.57 \mathrm{~B}$ & $\$ 8.0 \mathrm{~B}$ \\
& & & & \\
FY96 Capital & $\$ 5.8 \mathrm{~B}$ & $\$ 6.13 \mathrm{~B}$ & $\$ 5.8 \mathrm{~B}$ & $\$ 6.13 \mathrm{~B}$ \\
FY97 Capital & & & & \\
& $\$ 2.53 \mathrm{~B}$ & $\$ 2.67 \mathrm{~B}$ & $\$ 2.53 \mathrm{~B}$ & $\$ 2.68 \mathrm{~B}$ \\
\hline Total Capital & & & & \\
\hline
\end{tabular}


Table 7. Assumed Component Unit Costs

Component of Cost

Assumed Value

$\begin{array}{llll}\text { Arch } 1 & \text { Arch } 2 & \text { Arch } 3 & \text { Arch } 4\end{array}$

Planning and Engineering

$$
<==10 \% \text { of project cost }==>
$$$$
<==\$ 50 \mathrm{~K} / \mathrm{mi}==>
$$

Traffic Sensors

Signals and Controllers

Variable Message Signs

Beacons

$\mathbf{0}$

$<==\$ 40 \mathrm{~K} /$ int $==>$

$<==\$ 40 \mathrm{~K} / \mathrm{mi}==>$

Radio

$\$ 15 \mathrm{~K} / \mathrm{mi} \quad \$ 15 \mathrm{~K} / \mathrm{mi} \quad \$ 15 \mathrm{~K} / \mathrm{mi} \quad 0$

Communication Lines

Computer Hardware

$<==\$ 150 \mathrm{~K} / \mathrm{mi}==>$

$\$ 125 \mathrm{~K} / \mathrm{mod} \$ 125 \mathrm{~K} / \mathrm{mod} \$ 175 \mathrm{~K} / \mathrm{mod} \quad \$ 150 \mathrm{~K} / \mathrm{mod}$

Computer Software

$<==\$ 300 \mathrm{~K} / \bmod ==>$

Staff

Building Space

$<==\$ 400 \mathrm{~K} / \mathrm{yr}==>$

$<==\$ 200 \mathrm{~K} / \mathrm{mod}==>$ 


\section{References and Data Sources}

We are especially grateful to our consultants, Craig Gardner of JHK Associates, and Phil Tarnoff of Farradyne Systems.

Alternative IVHS Architectures. Working Paper 91-W-00512, The MITRE Corporation, 1991.

Economic Issues Associated with the Definition of Public/Private Roles in the

Deployment of IVHS. Farradyne Systems, Inc., 1992.

Operational Procedures and Strategies Conceptual Design Report. JHK \& Associates, (for CalTrans District 4 and San Francisco Bay area) 1992.

Congestion Avoidance and Reduction for Autos and Trucks (CARAT). Project proposal for Charlotte, NC prepared by North Carolina Department of Transportation.

The Los Angeles Autoniated Traffic Surveillance and Control (ATSAC) System. Edwin Rowe, 1990.

Southern Districts Traffic Operation Systems Communication Study. Fluor Daniel (for CalTrans) 1991.

Scope, Feasibility and Cost of a Dynamic Route Guidance System Demonstration. Final report for the ADVANCE project, prepared by University of Illinois-Chicago Urban Transportation Center, Northwestern Transportation Center and Motorola, 1990.

\section{Background sources}

We would also like to acknowledge the Colorado Department of Transportation, the Delaware Department of Transportation, the Florida Department of Transportation, the staff of the INFORM project, the Maryland Department of Transportation, the Michigan Department of Transportation, the Tennessee Department of Transportation, the staff of the TRANSCOM project, the Virginia Department of Transportation, Castle Rock Consultants, the Texas State Department of Highways and Public Transportation and the Greenville SC Planning Commission, who also provided useful and informative reports and other resources for our study. 
We will begin by defining the individual elements of cost of implementing ATMS in the largeat 75 metropolitan areas, the characteristics of the areas themselves, and the dependencies of the elements on size, miles, or some other characteristic.

Estimated cost for each area will then be computed by summing the element conts for that area Estimated total cost of implementation will then be computed by summing the area costs.

Data about the individual areas will be stored and malntained external to this model and read into the computation when the model is executed. Some values are experts' eathmates, and derived values are assumed explicitly in the model. In this report, all available metropolitan area data are utillbed. The data will be stored outulde the model.

\section{INDEX DEFINITIONS}

Let the metropolitan areas be indexed by 'I', where

$$
\begin{aligned}
& i:=0.74 \text { and the areas are those identified in the } \\
& 1990 \text { census (e.g., New York, the largest, is area 0), }
\end{aligned}
$$

and let the cout elements by indered by 'J', where

$$
j:=0 . .11
$$

element 0 bs planning and engincering cost,

element 1 is sensor cost,

element 2 is alonal coot,

element 3 is variablo-measage sign costh

element 4 is beacon cort (which will be 0 in some archiltectures),

e'ement 5 is radio cost (which will be 0 in some architecturea),

element 6 b communication 'cabling' cost,

element 7 b computer hardware original cost,

element 8 is computer software origieal cost,

element 9 is stafi coet,

element $10 \mathrm{~b}$ cost of providing building apsece, and

element 11 is 'other' - to be defined - cost.

Costs are atated as per-mile or other appropriate unit costs, and then multiplled by the appropriate factor to calculate element coet for a particular area. 


\section{READ IN AREA CHARACTERISTIC DATA}

Assume that some of the characteristics of the metropolitan areas are stored in the rows of an ASCU Nile (named "ascidat.prn"), ordered by size rank, with each row of the file containing the area's population, number of freeway miles, number of arterial milles, number of signalized intersections, and adjustment factors for geographic location and curreat status of advanced tramic management planning or implementation. We access the file with 'READ' statements. For programming convenience, we'll define a data matrix for temporary storage, read into it, and pick out the individual characteristic data for further use.

Index the characteriatics by 'k', where

$k:=0 . .5$

Then data : = READPRN (asciidat)

Pop $_{1}:=$ data $_{1,0}$

Population, in thousands Modules $_{1}:=$ ceil $\left(\frac{\text { Pop }_{1}}{10^{3}}\right)$.

Number of TMC modules

FwyMi $_{1}:=$ data $_{1.1}$

Number of freeway miles

MinArtMi, : data $_{1,2}$

Number of arterial miles

AugArtini $_{1}:=\operatorname{data}_{(i, 2)}, 5$

Number of (augmented) arterial miles

Aug := 0 'Aug' lets us consider the two cusean.

TotMil $:=\operatorname{If}($ Aug $>0, F$ wyMi + AugArtMi, FwyMi + MinArtmi $)$

Intrsect $:=$ if $\left(\right.$ Aug $>0$, data $_{1,3}, 5$, data $\left._{1,3}\right)$

Number of intersections controlled

Geofactor $_{1}:=$ datg $_{1,4}$

Factor to adjust for difroring construction coats by climate and difiering internection density

Statusfactor $_{1}:=$ data $_{1,5}$

Factor to adjust for planning and implementation already exinting 


\section{DEFINE UNIT COSTS FOR ARCHITECTURE 1}

Costs will be given in $\mathbf{K}$ (thousands), $M$ (millions), or B (billions)

$$
\text { where } K=1 \quad M=1000 \cdot K \text { and } B=1000 \cdot 11
$$

\begin{tabular}{|c|c|}
\hline SignalCostPerintrsect $=40 \cdot \mathrm{K}$ & SensorCostPerMile $=50 \cdot \mathrm{K}$ \\
\hline Fr old $:=0.5$ & VMSCostPerMile $=40 \cdot \mathrm{K}$ \\
\hline BeaconCostPerMile $=0 . K$ & RadioCostPerMile $=15 \cdot \mathrm{K}$ \\
\hline CommCostPerMile : $=150 \cdot \mathrm{K}$ & CompHdweCostPerModule $=125 \cdot \mathrm{K}$ \\
\hline SoftwelnstanceCostPerMile $:=2 \cdot \mathrm{K}$ & StaffCostPerModule $:=400 \cdot K$ \\
\hline BuildingCostPerModule $:=200 \cdot \mathrm{K}$ & SottweOrigCostPerModule $:=300 \cdot \mathrm{K}$ \\
\hline OtherCostPerMile : & SoftwoUpdateCostPerModule $:=15 \cdot \mathrm{K}$ \\
\hline
\end{tabular}

(will be added as needed)

\section{CALCULATE COST ELEMENTS FOR EACH AREA}

The elements of cost, as linted above, can then be caleulated for each spectice area.

$$
\begin{aligned}
& \text { ElementCost }_{(1,0)}:=0 \quad \text { (will add in } 2010 \% \text { after calculation of capital cont) } \\
& \text { ElementCost },:=\text { SensorCostPerMile. StatusFactor, } \text { GeoFactor }_{1} \cdot \text { TotMi, }_{1} \cdot K
\end{aligned}
$$

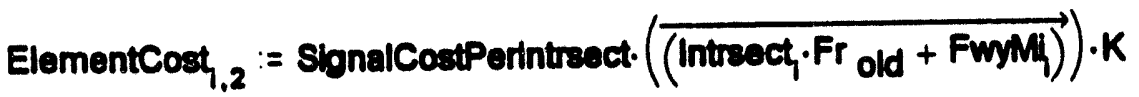

$$
\begin{aligned}
& \text { ElementCost, }:=\text { VMSCostPerMile. FusMi, } \cdot \text { GeoFactor }, \text { Status Factor }, K \\
& \text { ElementCost },:=\text { BeaconCostPerMile }[\text { (Fwyil }+ \text { MinArtMi }) \cdot K] \\
& \text { ElementCost, }:=\text { RadioCostPerMille. FwyMt } \cdot K \\
& \text { ElementCost }, 0:=\text { CommCostPerMile. TotMi, GeoFactor } \cdot K \\
& \text { ElementCost }, 7:=\text { CompHdweCostPerModule.Modules, } K
\end{aligned}
$$

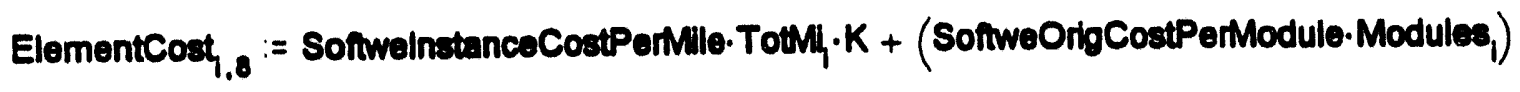

$$
\begin{aligned}
& \text { ElementCost, }:=\text { StaffCostPerModule } \cdot \text { Modules, } \cdot K \\
& \text { ElementCost }_{1,10}:=\text { BulldingCostPerModule } \cdot \text { Modules, } \cdot K \\
& \text { ElementCost }_{(1,11)}:=\text { OtherCostPerMile.K }
\end{aligned}
$$


With elements of cost and characteristics of the individual areas jefined, total estimated cost for each area can be calculated by summing over the elements of cost.

$$
\begin{aligned}
& \text { InstalledCost }:=\sum_{j} \text { ElementCost }_{1, j}(j<9) \\
& \text { CapitalCost }=\text { InstalledCost }-1.1 \quad \text { ElementCost }_{(1,0)}=\text { InstalledCost }, \cdot 1_{1}
\end{aligned}
$$

Considerting engibeering as $10 \%$ of capital cost

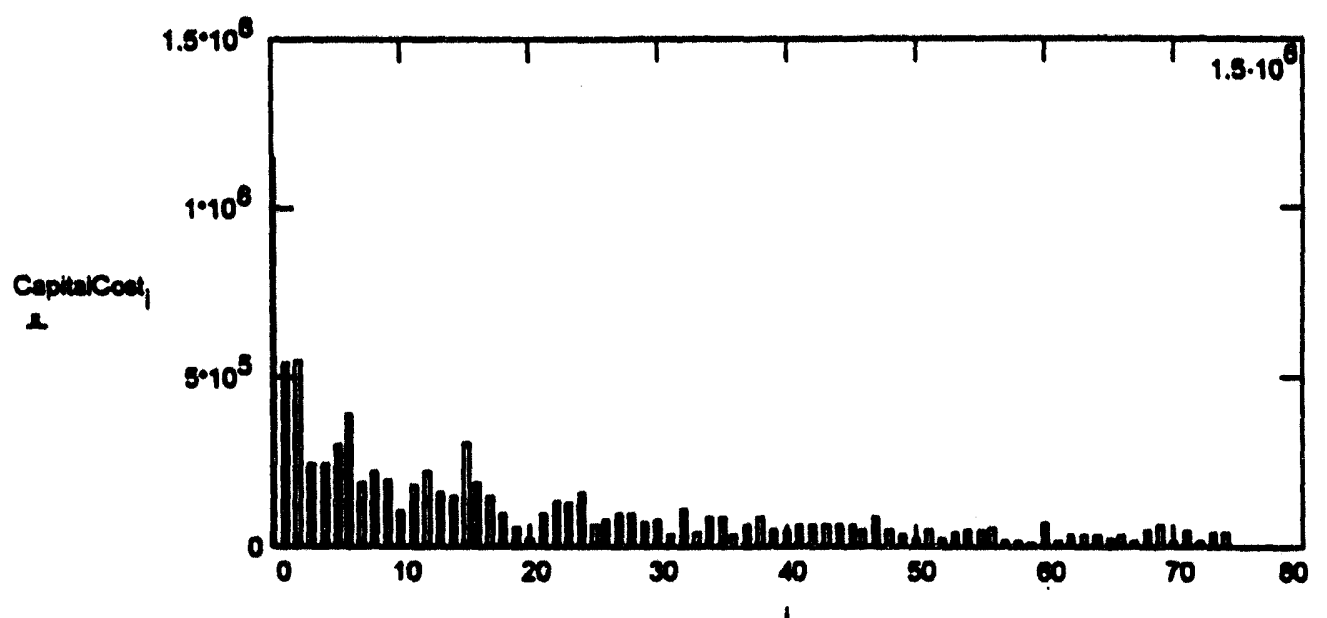

\section{SUM CAPITAL COSTS BY SIZE OF ARJAS}

To sum costs for the largeat 9 metropolitan arese, (pop > 4M) we sum from 0 to 8;

$$
\text { LargeAreaCost : }=\sum_{1} \text { CapttalCost, }(i<9)
$$

LargeAreaCost $=3.81 \cdot B$

Similarly,

$$
\begin{aligned}
& \text { MediumLargeAreaCost : }=\sum_{i} \text { CapitalCost, } \cdot(i>8) \cdot(i<22) \\
& \text { MediumLargeAreaCost }=1.91 \cdot B \\
& \text { MediumSmallAreaCost }:=\sum_{1} \text { CapitalCost, } \cdot(i>21) \cdot(i<41) \\
& \text { SmallAreaCost }:=\sum_{1} \text { CapitalCost } \cdot(1>40) \quad \text { SediumSmallAreaCost }=1.51 \cdot B \\
& \text { SmallAreaCost }=1.33 \cdot B
\end{aligned}
$$


ElementCapitalCost $_{j}=\sum_{i}$ ElementCost $_{(i, j)}$

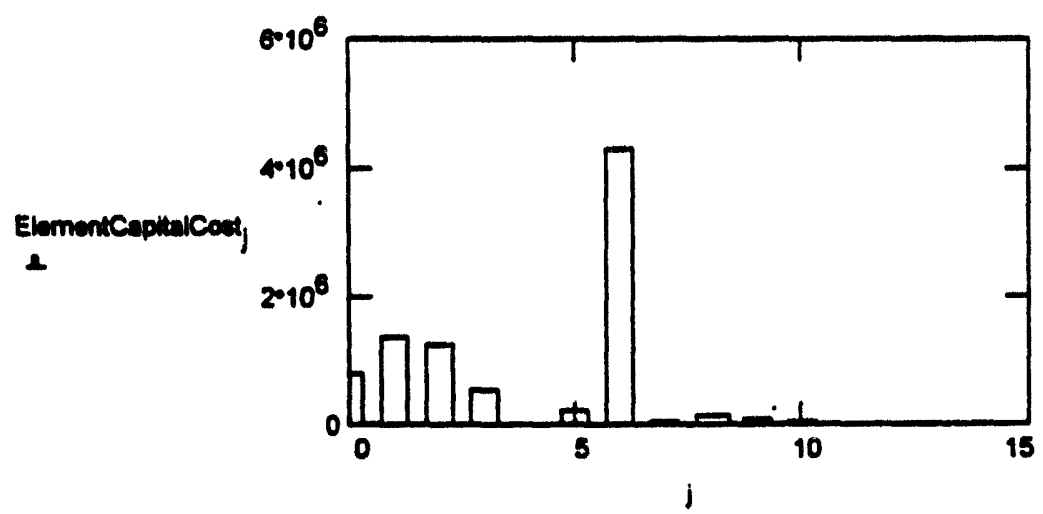

For reference, remember that

element 0 is planning and engineering cost element 1 is reusor cost, element 2 is signal cost, element 3 is variable-measage sign cost, element 4 is bescou cost, element 5 b radio cost, element 6 is communication 'cabling' cost, element 7 is computer hardware orighed cost, element 8 is computer software ortginal cont, element 9 is itufi costh element $10 \mathrm{~b}$ cost of providing bullding apace, and element 11 bs 'other' - to be defined - cout.

\section{SUM CAPITAL COSTS FOR ALL METROPOUTTAN AREAS}

To estimate the grand total capital cost for all areas, we simply sum the estimated costs for every ares.

$$
\text { GrandTotalCapital : }=\sum_{1} \text { CapitalCost }
$$

With the atated asoumptions, the grand total b

GrandTotalCapital $=\mathbf{8 . 5 7} \cdot \mathrm{B}$ 
To estimate costs for operations and maintenance, we take $10 \%$ of the capital cost for an area for maintenance - and add the projected other recurring costs (for example, salary and oraining).

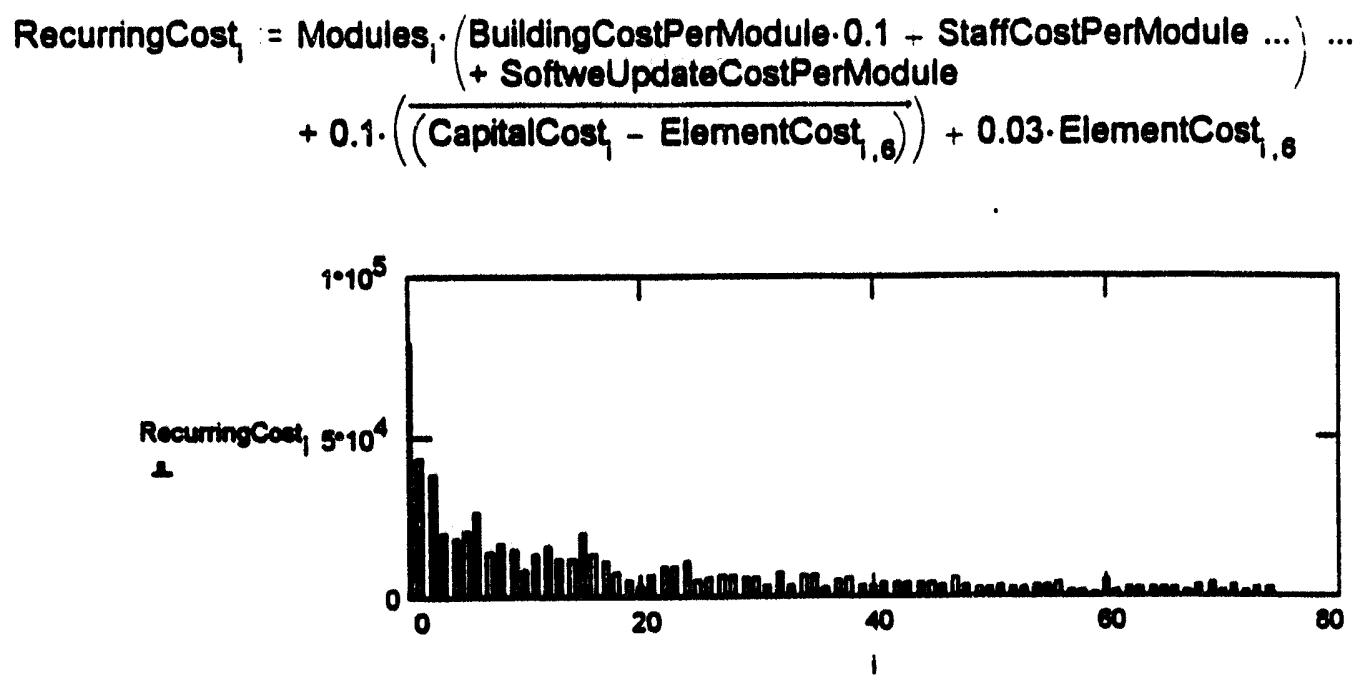

\section{SUM RECURRING COSTS BY SIZE OF AREA}

$$
\begin{aligned}
& \text { LargeAreaRecurringCost : }=\sum_{i} \text { RecurringCost } \cdot(i<9) \\
& \text { LargeAreaRecurringCost }=275.77 \cdot \mathrm{M} \\
& \text { MediumLargeAreaRecurringCost : = } \sum_{i} \text { RecurringCost } \cdot(i>8) \cdot(i<22) \\
& \text { MediumLargeAreaRecurringCost }=143.17 \cdot M \\
& \text { MediumSmallAreaRecurringCost := } \sum_{i} \text { RecurringCost. } \cdot(i>21) \cdot(i<41) \\
& \text { MediumSmallAreaRecurringCost }=114.42 \cdot M \\
& \text { SmallAreaRecurringCost : }=\sum_{i} \text { RecurringCost } \cdot(1>40) \\
& \text { SmallAreaRecurringCost }=102.61 \cdot M \\
& \text { TotalRecurringCost : }=\sum_{i} \text { RecurringCost } \\
& \text { TotalRecurringCost }=0.64 \cdot B
\end{aligned}
$$


To estimate the distribution of ATMS implementation costs over the next five years, we begin by assuming spending proflies for projects. We assume that large projects take longer than amall projects, but that the shapes of the apending curves are simllar. We further assume that large areas are farther along in the ATMS process, and bence can begin sooner. For convenience, we store the proflies in a separate ASCII Ne called TIMEDIST.PRN', where each row of the file contains the fraction of total project cost that is apent during a particular 3-month interval and the 20 rows therefore represent spending profiles for five years. Again, we access the file with a 'READPRN' statement and store the data in a temporary array.

$$
\text { TimeCoeffs : = READPRN (timedist prn) }
$$

$$
\begin{aligned}
& \text { Inclex the data by quarters, where } \\
& q \text { tr }=0 . .19 \\
& \text { and index the area strea by kn where } \\
& k=0 . .3 \quad\left(0^{\prime}\right. \text { is largeat) } \\
& \text { Recall that } \\
& K=1 \\
& M=1 \cdot 10^{3} \cdot K \\
& B=1 \cdot 10^{3} \cdot M \\
& \text { LargeAreaCost }=3.81 \cdot B \\
& \text { QurCost }_{q r, 1}:=\text { TimeCoeffs }_{q r, 1} \cdot \text { MediumLargeAreeCost } \\
& \text { QtrCost }_{q r, 2}:=\text { TimeCoeffs }_{q,, 2} \cdot \text { MediumSmallAredCost } \\
& \text { QtrCost }_{q, 3}:=\text { TimeCoeffs }{ }_{q t, 3} \text { SmallAreaCost }
\end{aligned}
$$

For ezample,

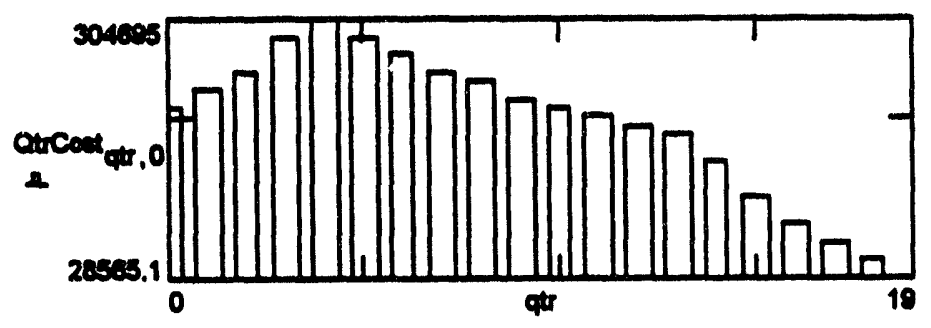

To get total implementation coat by year, we sum over the area costs and then sum the quarters in groups of four.

$$
\text { QtrSum }_{\phi r}:=\sum_{k} \text { QtrCost }_{\phi, k}
$$




$$
\text { Let years be indexed by } \quad y r=0.4
$$

$$
\begin{aligned}
& \text { AnnualCost }_{0}=\sum_{q t r} \text { QtrSum }_{\text {qtr }} \cdot(\text { qtr }<4) \\
& \text { AnnualCost }_{0}=0.97 \cdot B \\
& \text { AnnualCost }_{1}:=\sum_{\text {qt }} \operatorname{QtrSum}_{\text {qr }} \cdot(\text { qtr }>3) \cdot(\text { qtr }<8) \\
& \text { AnnualCost }_{1}=2.03-8 \\
& \text { AnnualCost }_{2}:=\sum_{q t r} \text { QtrSum }_{\phi r} \cdot(\text { qtr }>7) \cdot(\text { qtr }<12)
\end{aligned}
$$$$
\text { AnnualCost }_{2}=2.65 \cdot \mathrm{B}
$$$$
\text { AnnualCost }_{3}:=\sum_{\text {qtr }} \text { QtrSum }_{\text {qri }} \cdot(\text { qtr }>11) \cdot(\text { qtr }<16)
$$$$
\text { AnnualCost }_{3}=2.03 \cdot B
$$

$$
\text { AnnualCost }_{4}:=\sum_{q t r} \text { QtrSum }_{\text {qr }} \cdot(q t r>15)
$$

$$
\text { AnnualCost }_{4}=0.89 \cdot B
$$

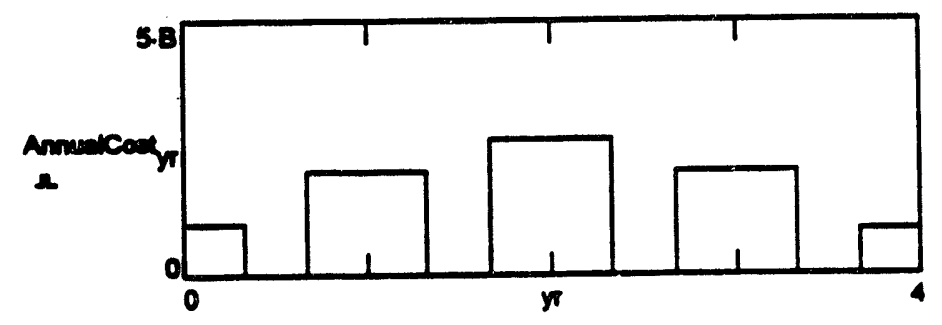

As a checkn,

$$
\begin{aligned}
\text { SumOfCost : }=\sum_{y r} \text { AnnualCost }_{y} \quad \text { SumOfCost } & =8.57 \cdot B \\
\text { GrandTotalCapital } & =8.57 \cdot B
\end{aligned}
$$




\section{APPENDEX}

Cost Estimates for ATMS Implementation: Computational Procedures

We will begin by defining the individual elements of cost of implementing ATMS in the largest 75 metropolitan areas, the characteriatics of the areas themselves, and the dependencies of the elements on stze, miles, or some other characteristic.

Estimated cost for each area will then be computed by summing the element coats for that area. Eatimated total cost of implementation will then be computed by summing the area costs.

Data about the individual areas will be stored and maintained external to this model and read into the computation when the model is exeented. Some values are experts' estimates, and derived values are asaumed explicttly in the modeh. In this report, all avallable metropolitan ares data are utilized. The data will be stored outuide the model.

\section{INDEX DEFINITIONS}

Let the metropolitan areas be indexed by 'i', where

$$
\begin{aligned}
& i=0.74 \text { and the areas are those identified in the } \\
& 1990 \text { census (e.g., New York, the largeat, is area 0), }
\end{aligned}
$$

and let the coat elements by indered by 'J', where

$$
\text { j : }:=0 . .11
$$

element 0 is planning and engineering cost,

element 1 is ecasoor cost,

clement 2 bs slignal coot,

element 3 b variable-measage sign cost,

element 4 is beacen cost (which will be 0 in some architectures),

element 5 is radio coet (which will be 0 in some arehitecturss),

element 6 b communication 'cabling' cont,

element 7 is computer hardware orifinal couth,

element 8 b computer eoftware original costh

element 9 b atafi cost,

element $10 \mathrm{~b}$ cont of providing building apace, and

element 11 is 'other' - to be defined - cost.

Coats are stated as per-mile or other appropriate unit costs, and then multiplied by the approp: ante factor to calculate element cost for a particular area. 


\section{READ IN AREA CHARACTERISTIC DATA}

Assume that some of the characteristics of the metropolitan areas are stored in the rows of an ASCD Tle (named "asciidat.prn"), ordered by size rantw, with each row of the file containing the area's population, number of freeway miles, number of arterial miles, number of signalized intersections, and adjustment factors for geographic location and current status of advanced trafic management planning or implementation. We access the file with 'READ' statements. For programming convenience, we'Ul define a data matrix for temporary storage, read into it, and pick out the individual characteriatic data for further use.

Inder the characteristica by ' $k$ ', where

$$
k:=0 . .5
$$

Then

$$
\text { data := READPRN( asciidat) }
$$

Pop $_{1}=$ data $_{1,0}$

$$
\text { Modules, }_{1}=\operatorname{ceil}\left(\frac{P_{0 p_{1}}}{10^{3}}\right)
$$

Fuymi $_{1}:=$ data $_{1,1}$

MinArtMi, := data 1.2

AugArtmil $_{1}:=\operatorname{data}_{(1,2)} .5$
Population, in thousands

Number of TMC modules

Number of freeway miles

Number of arterial miles

Number of (augmented) arterial miles

Aug := 1 'Aug' lets us consider the two casesm

TotMi $:=$ if (Aug> 0, FWyMi + AugArtMi, FWYMi + MinArtMi $)$

Intrsect, $:=$ if $\left(\right.$ Aug $>0$, data $_{1,3}, 5$, data $\left._{1,3}\right)$

Number of intersections controlled

GeoFactor $_{i}:=$ data $_{1,4}$

Factor to adjust for difiering construction costa by climate and difiering internection density

StatusFactor $_{1}:=$ data $_{1,5}$

Factor to adjust for planuing and

implementation already exinting 
Costs will be given in $\mathbf{K}$ (thousands), $M$ (millions), or $\mathbf{B}$ (billions)

$$
\text { where } K=1 \quad M=1000 \cdot K \text { and } B=1000 \cdot M
$$

$\begin{array}{ll}\begin{array}{l}\text { SignalCostPerintrsect }=40 \cdot \mathrm{K} \\ \mathrm{Fr} \text { old }=0.5 .\end{array} & \begin{array}{l}\text { SensorCostPerMile }=50 \cdot \mathrm{K} \\ \text { BeaconCostPerMile }=0 \cdot \mathrm{K}\end{array} \\ \text { CommCostPerMile }:=150 \cdot \mathrm{K} & \begin{array}{l}\text { RadioCostPerMile }=15 \cdot \mathrm{K} \\ \text { CompHdweCostPerModule }=125 \cdot \mathrm{K}\end{array} \\ \text { SoftweinstanceCostPerMile }:=2 \cdot \mathrm{K} & \text { StaffCostPerModule }=400 \cdot \mathrm{K} \\ \text { BuildingCostPerModule }=200 \cdot \mathrm{K} & \text { SoftweOrigCostPerModule }:=300 \cdot \mathrm{K} \\ \text { OtherCostPerMile }:=0 & \text { SoftweUpdateCostPerModule }=15 \cdot \mathrm{K} \\ \text { (will be added as needed) }\end{array}$

\section{CALCULATE COST ELEMENTS FOR EACH AREA}

The elements of cost, as linted above, can then be calculated for each apecific area.

$$
\begin{aligned}
& \text { ElementCost }_{(i, 0)}:=0 \quad \text { (will add in as } 10 \% \text { after calculation of capital cost) } \\
& \text { ElementCost } t_{1,1}:=\text { SensorCostPerMile.StatusFactor }, \cdot \text { GeoFactor }_{i} \cdot \text { TotMi }_{i} \cdot \mathrm{K} \\
& \text { ElementCost, }_{1,2}:=\text { SignalCostPerintrsect } \cdot\left(\overrightarrow{\left(\text { Intrsect, } \cdot \mathrm{Fr}_{\text {old }}+\mathrm{FwyM}_{\mathrm{i}}\right)}\right) \cdot \mathrm{K} \\
& \text { ElementCost, }:=\text { VMSCostPerMile }_{1,3} \text { FuMMi }_{i} \cdot \text { GeoFactor }_{i} \text { StatusFactor }, \cdot K \\
& \text { ElementCost }_{1,4}:=\text { BeaconCostPerMile } \cdot[(\text { FwMMi }+ \text { MinArtMi }) \cdot K] \\
& \text { ElementCost }_{1,5}:=\text { RadioCostPerMile. FwMili } K
\end{aligned}
$$

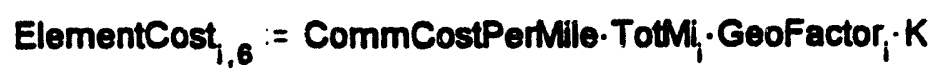

$$
\begin{aligned}
& \text { ElementCost },:=\text { CompHdweCostPerModule.Modules }, \cdot K
\end{aligned}
$$

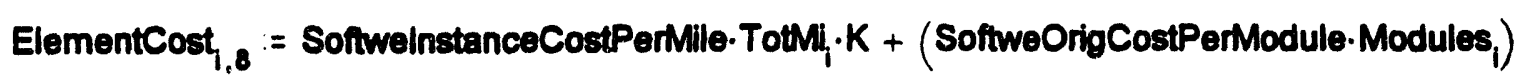

$$
\begin{aligned}
& \text { ElementCost }, 0=\text { StaffCostPerModule } \cdot \text { Modules }, \cdot K \\
& \text { ElementCost } 1,10 \text { := BuildingCostPerModule.Modules, } \cdot K \\
& \text { ElementCost }_{(1.11)}:=\text { OtherCostPerMile.K }
\end{aligned}
$$


With elements of cost and characteristics of the individual areas defined, total estimated cust for each area can be calculated by summing over the elements of cost.

InstalledCost $=\sum_{j}$ ElementCost $_{i, j}(j<9)$

CapitalCost $_{1}=$ InstalledCost $_{1} 1.1 \quad$ ElementCost $_{(1,0)}=$ InstalledCost $_{j} \cdot 1$

Considering engineering as $10 \%$ of capital cost

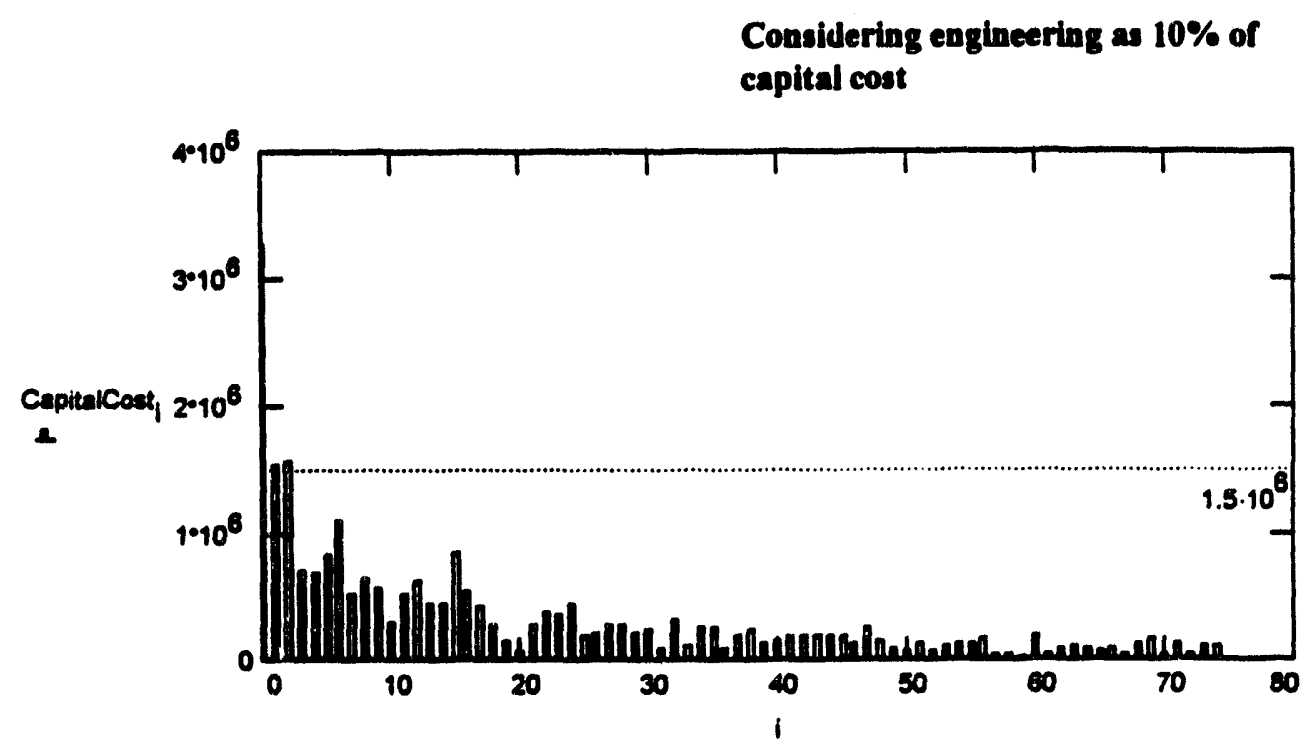

\section{SUM CAPITAL COSTS BY SIZE OF AREAS}

To sum conts for the largest 9 metropolitan areas, (pop > 4M) we sum from 0 to 8;

$$
\text { LargeAreaCost := } \sum_{i} \text { CapitalCost, }(i<9)
$$

LargeAreaCost $=10.89 \cdot B$

Similarly,

$$
\begin{aligned}
& \text { MediumLargeAreaCost }:=\sum_{i} \text { CapitalCost, }(i>8) \cdot(i<22) \\
& \text { MediumLargeAreaCost }=5.45 \cdot B \\
& \text { MediumSmallAreaCost }:=\sum_{i} \text { CapitalCost }, \cdot(i>21) \cdot(i<41)_{\text {MediumSmallAreaCost }=4.31 \cdot B} \\
& \text { SmallAreaCost }:=\sum_{i} \text { CapitalCost } \cdot(i>40) \\
& \text { SmallAreaCost }=3.8 \cdot B
\end{aligned}
$$




$$
\text { ElementCapitalCost }_{1}=\sum_{1} \text { ElementCost }_{(1, j)}
$$

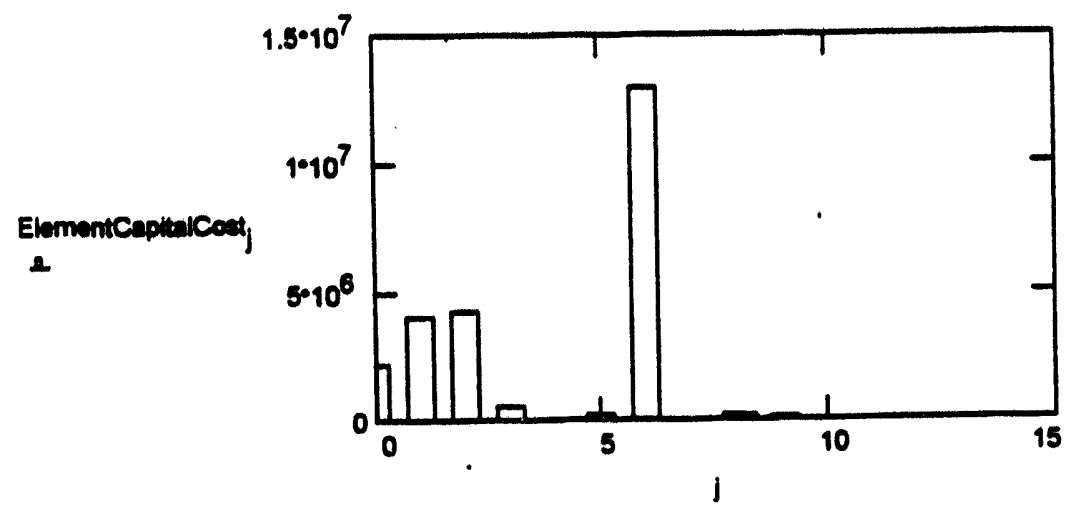

For reference, remember that

element 0 is planning and engineering cost

element 1 is sensor costh

element 2 is signal cost,

element 3 is variable-mesange sign cost,

element 4 is bescon cost,

element 5 is radio cont,

element 6 is communication 'cabling' cost,

element 7 is computer hardware original costh

element 8 is computer software original cost,

element 9 is atafir cost,

element 10 is cost of providing building space, and

element 11 b 'other' - to be defined - cost.

\section{SUM CAPITAL COSTS FOR ALL METROPOLTAN AREAS}

To eatimate the grand total capital cost for all areas, we simply sum the eatimated costs for every area.

$$
\text { GrandTotalCapital : }=\sum_{1} \text { CapitalCost }
$$

With the stated assumptions, the grand total b

GrandTotalCapital $=24.46 \cdot B$ 


\section{SUM RECURRING COSTS FOR INDIVDUAL METROPOLTTAN AREAS}

To estimate costs for operations and malatenance, we take $10 \%$ of the capital cost for an area for maintenance - and add the projected other recurring costs (for example, salary and tralaing).

RecurringCost $=$ Modules $_{i} \cdot\left(\begin{array}{l}\text { Building CostPerModule } \cdot 0.1-\text { StaffCostPerModule } \ldots ; \\ + \text { SoftwoUpdateCostPerModule }\end{array} ;\right.$

$+0.1 \cdot\left(\overline{\left(\text { CapitalCost }_{1} \text { - ElementCost }\right.}, \overline{1, j}\right)+0.03$. ElementCost 1.0

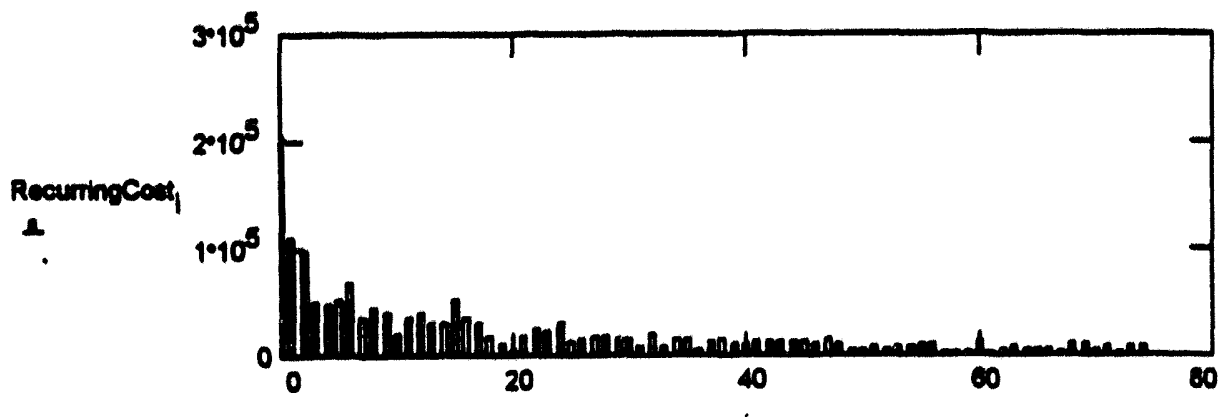

\section{SUM RECURRING COSTS BY SIZE OF AREA}

$$
\begin{aligned}
& \text { LargeAreaRecurringCost : }= \sum_{i} \text { RecurringCost } \cdot(i<9) \\
& \text { LargeAreaRecurringCost }=709.53 \cdot M \\
& \text { MediumLargeAreaRecurringCost : } \sum_{i} \text { RecurringCost } \cdot(i>8) \cdot(i<22) \\
& \text { MediumLargeAreaRecurringCost }=367.31 \cdot M \\
& \text { MediumSmallAreaRecurringCost : }= \sum_{i} \text { RecurringCost, }(i>21) \cdot(i<41) \\
& \text { MediumSmallAreaRecurringCost }=288.9 \cdot M
\end{aligned}
$$$$
\begin{gathered}
\text { SmallAreaRecurringCost : }=\sum_{i} \text { RecurringCost } \cdot(1>40) \\
\text { SmallAreaRecurringCost }=258.2 \cdot \mathrm{M}
\end{gathered}
$$

TotalRecurringCost : $=\sum_{1}$ RecurringCost

$$
\text { TotalRecurringCost }=1.62 \cdot B
$$


To eatimate the distribution of ATMS implementation costs over the next five yearn, we begln by assuming spending proflies for projects. We assume that large projects take longer than small projects, but that the shapes of the speading curves are simllar. We further assume that large areas are farther along in the ATMS process, and hence can begin sooner. For convenience, we store the proflies in a separate ASCII Nle called 'TDMEDIST.PRN', where each row of the the contains the fraction of total project cost that is apent during a particular 3-month interval and the 20 rows therefore represent spending proflles for five yearn. Again, we access the file with a 'READPRN' statement and store the data in a temporary array.

$$
\begin{aligned}
& \text { TimoCoeffs : = READPRN (Umedist prn) } \\
& \text { Index the data by quartern, where } \\
& \text { qur }=0 . .19 \\
& \text { and inder the area stees by } k_{n} \text { where } \\
& k=0 . .3 \quad \text { ('0' is largeat) } \\
& \text { Recall that } K=1 \quad M=1 \cdot 10^{3} \cdot K \quad B=1 \cdot 10^{3} \cdot M \\
& \text { LargeAreaCost }=10.89 \cdot B
\end{aligned}
$$

ModiumLargeAreaCost $=5.45 \cdot B$

MediumSmallAreaCost $=4.31 \cdot B$

SmallAreaCost $=3.8 \cdot 8$

Then

$$
\begin{aligned}
& \text { QurCost }_{\text {qr }, 0}=\text { TimeCoeffs }_{\text {qu }, 0} \cdot \text { LargeAreaCost }
\end{aligned}
$$

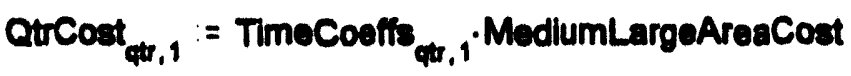

$$
\begin{aligned}
& \text { QtrCost }_{\phi t, 2}=\text { TimeCooffs }{ }_{q, 2} \cdot \text { MediumSmallAreaCost } \\
& \text { QtrCost }_{q r, 3}:=\text { TimeCoeffs }_{q r, 3} \cdot \text { SmallAreaCost }
\end{aligned}
$$

For examples

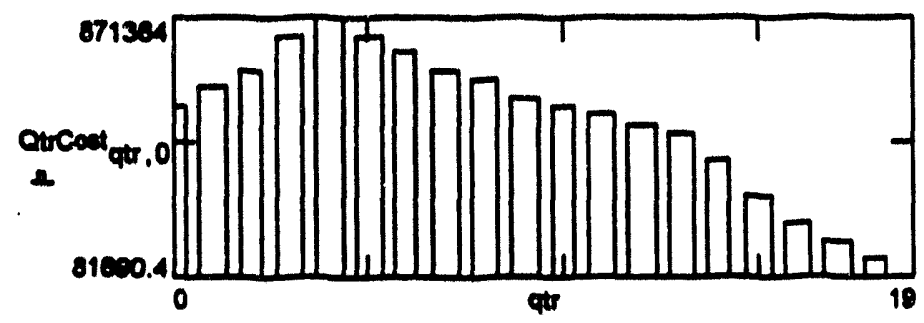

To get total implementation cost by year, we sum over the area costs and then sum the quarters in groups of four.

$$
\text { QtrSum }_{\text {qu }}:=\sum_{k} \text { QtrCost }_{\phi t, k}
$$


Let years be indexed by $\quad y r=0.4$

$$
\begin{aligned}
& \text { AnnualCost }_{0}=\sum_{\text {qtr }} \text { QtrSum }_{\text {qur }} \cdot(\text { qtr }<4) \\
& \text { AnnualCost } t_{0}=2.78 \cdot B \\
& \text { AnnualCost }_{1}:=\sum_{q t r} \operatorname{QtrSum}_{q t r} \cdot(q t r>3) \cdot(q t r<8) \\
& \text { AnnualCost }_{1}=5.79 \cdot \mathrm{B} \\
& \text { Annualcost }_{2}:=\sum_{q t r} \text { Qtrsum }_{q u} \cdot(q t r>7) \cdot(q t r<12) \\
& \text { AnnualCost } 2=7.56 \cdot B \\
& \text { AnnualCost }_{3}=\sum_{\text {qtr }} \text { QtrSum }_{\text {qtr }} \cdot(\text { qtr }>11) \cdot(\text { qtr }<16) \\
& \text { AnnualCost }_{3}=5.8 \cdot B \\
& \text { AnnualCost }_{4}:=\sum_{\text {qtr }} \text { QtrSum }_{\phi i} \cdot(\text { qtr> 15) }
\end{aligned}
$$

AnnualCost $_{4}=2.53 \cdot \mathrm{B}$

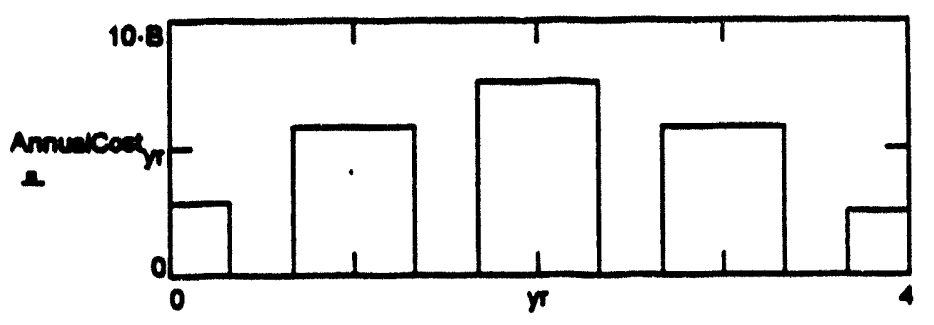

As a cheekn,

$$
\begin{aligned}
\text { SumofCost }:=\sum_{y r} \text { AnnualCost }_{y r} \quad \text { SumOfCost } & =24.46 \cdot B \\
\text { GrandTotalCapital } & =24.46 \cdot B
\end{aligned}
$$


We will begin by defining the lodividual elements of cost of lomplowenting ATMS to the largent 75 motropolitan areas, the characteriatice of the areas themselves, and the dependencies of the -lemeats on sbe, milles, or some othor charactorlatic.

Eotlmated coot for each area will then bo computed by summing the dement coots for that area. Estimated total cost of Implementation will then be computed by summing the area coste.

Data about the individual areas with be storod and maintained external to this model and read bito the computation when the model b executed. Some values are experts' cotimatee, and dertved valuen are asaumed explieltly in the model. If thb report, all avallable metropoliten area data are utilleed. The data will be stored outelde the model.

\section{INDEX DEFINITONS}

Let the metropolitan areas be indezed by 'T', where

$$
\begin{aligned}
& 1=0.74 \text { and the areas are thoes ldenttined in the } \\
& 1990 \text { cenous (age. New York, the largeot, b area 0), }
\end{aligned}
$$

and let the cost eloments by leclexed by 'I', where

$$
J=0 . .11
$$

element 0 is planuing and englmeortes cooth,

element 1 ba sensor costh

clament 2 in algnal costh

element 3 b variable-measage alge coot,

-lement 4 is beacon cost (which will be 0 in some arebltecturen),

element $\mathrm{S}$ is radio cost (which will be $0 \mathrm{ln}$ some architecturew),

element 6 is communication 'eabling' cost,

element 7 b computer hardware origladel coot,

element 8 b computer software origlnal cout,

element 9 is staft cont,

element 10 is cont of providing building space, and

element 11 is 'other' - to be defined - coeth.

Coats are stated as per-mile or other appropriate unit costs, and then multiplied by the appropriate factor to calculate element cost for a particular area. 


\section{READ IN AREA CHARACTERISTIC DATA}

Assume that come of the charactortaties of the motropolitan aress are stored in the rows of an ASCI Whe (named "acelldatppre"), ordered by atee rankl, with each row of the flle containing the area's population, number of froway milien, number of artortal miles, number of algaalleed intersections, and adjustment factors for geographic location and curreat atatus of advancad trailic management planaine or implementation. We scewes tho It) whth 'RSAD' statementh. For programming coavenience, wo'll defime a data matris for temporary storage, read into it, and plek out the Individual characteriatic data for furtber uce.

Index the charactertatios by 'k', whore

$$
k:=\mathbf{0 . . 5}
$$

$$
\begin{aligned}
& \text { Then dat := READPRN (ascildat) }
\end{aligned}
$$

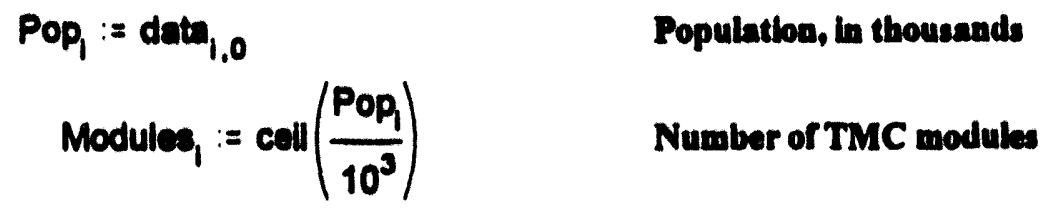

$$
\begin{aligned}
& \text { Fusig : }: \text { data }_{1,1} \quad \text { Number of freeway milles } \\
& \text { Minartim := data } 1,2 \quad \text { Number of arterial millee } \\
& \text { AugArtiky := data }(1,2) \cdot 5 \quad \text { Number of (augmented) arterial millem } \\
& \text { Aug : = } 0 \text { 'Aug' lets us conalder the two caseabos }
\end{aligned}
$$

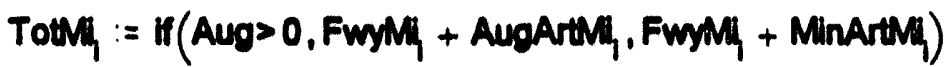

$$
\begin{aligned}
& \text { Intreect }:=I f\left(\text { Aug }_{0} 0, \text { data }_{1,3}, 5, \text { data }_{1,3}\right)
\end{aligned}
$$

Number of internectlons controlled

$$
\begin{aligned}
& \text { Geofactor }:=\text { data }_{1.4} \quad \text { Factor to adjuat for ditiering } \\
& \text { construction conts by climate and difrering } \\
& \text { internection denaity } \\
& \text { Statuafactor }_{1}=\text { data }_{1,8} \quad \text { Factor to adjuat for planning and } \\
& \text { implementation already exioting }
\end{aligned}
$$


DEFINE UNTT COSTS FOR ARCHITLCTURE 2

Costs will be given in $\mathbf{K}$ (thousands), $\mathbf{M}$ (millioas), or B (billiona)

$$
\text { where } K=1 \quad M=1000 \cdot K \text { and } B:=1000 \cdot M
$$

SignalCoutPerintreect $=40 \cdot \mathrm{K}$

$$
\text { Fr old : }=0.5
$$

BeaconCostPorMile : $=50 \cdot \mathrm{K}$

CommCostPerkille : $=150 \cdot K$

SoltwolnstancecostPerMile : $=2 \cdot K$

BuildingCostPorModule : $=200 \cdot \mathrm{K}$

OtherCostPorMila :=0

(will be addod as meeded)

\section{CALCULATE COST ELEMENTS FOR EACH AREA}

The elements of coat, as libted above, can then be calculated for each apectic area.

$$
\begin{aligned}
& \text { ElementCost }_{(1,0)}:=0 \quad \text { (will add in as } 10 \% \text { anter calculation of capital cost) } \\
& \text { ElementCost },:=\text { SensorCostPerMile. StatusFactor, } \text { GeoFactor }_{1} \text {-TotMi, } K
\end{aligned}
$$

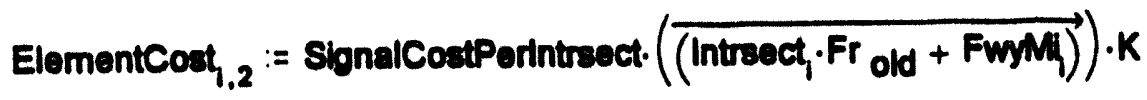

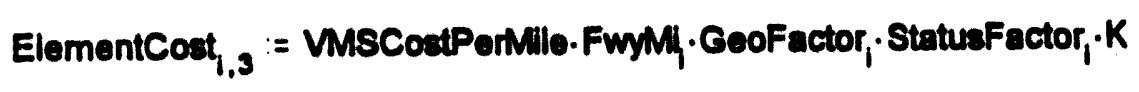

$$
\begin{aligned}
& \text { ElementCost },:=\text { BeaconCostPerMillo } \cdot[(\text { FwyMil }+ \text { MinArtMi }) \cdot K]
\end{aligned}
$$

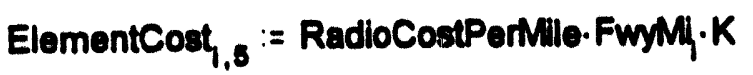

$$
\begin{aligned}
& \text { ElementCost, }:=\text { Comm CostPerMile. TotMi, } \cdot \text { GeoFactor }, K_{1} \\
& \text { ElementCost } 1,7=\text { CompHdwoCostPerModule } \cdot \text { Modules }_{1} K
\end{aligned}
$$

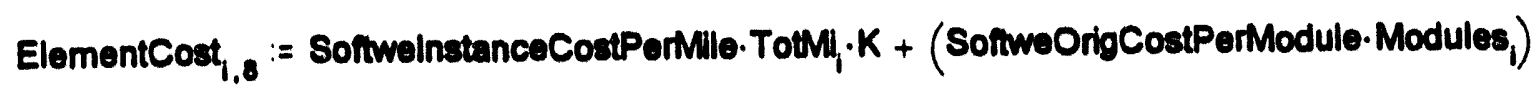

$$
\begin{aligned}
& \text { ElementCost } 1,0 \text { := StaffCostPerModule.Modules, } \cdot K \\
& \text { ElementCost } 1,10:=\text { BulldingCostPerModule } \cdot \text { Modules, } \cdot K \\
& \text { ElementCost }_{(1,11)}:=\text { OtherCostPerMile.K }
\end{aligned}
$$


With oloments of coat and characteristics of the individual areas defined, total eatimated cost for each area can be calculated by summing over the elements of cost.

InatalledCost, $:=\sum_{j}$ ElementCost $_{1, j}(j<\theta)$

CapltalCost $:=$ InstalledCost, $1.1 \quad$ ElementCost $(1,0):=$ InatalledCost $\cdot .1$

Conaldering engineering as $10 \%$ of capltal cost

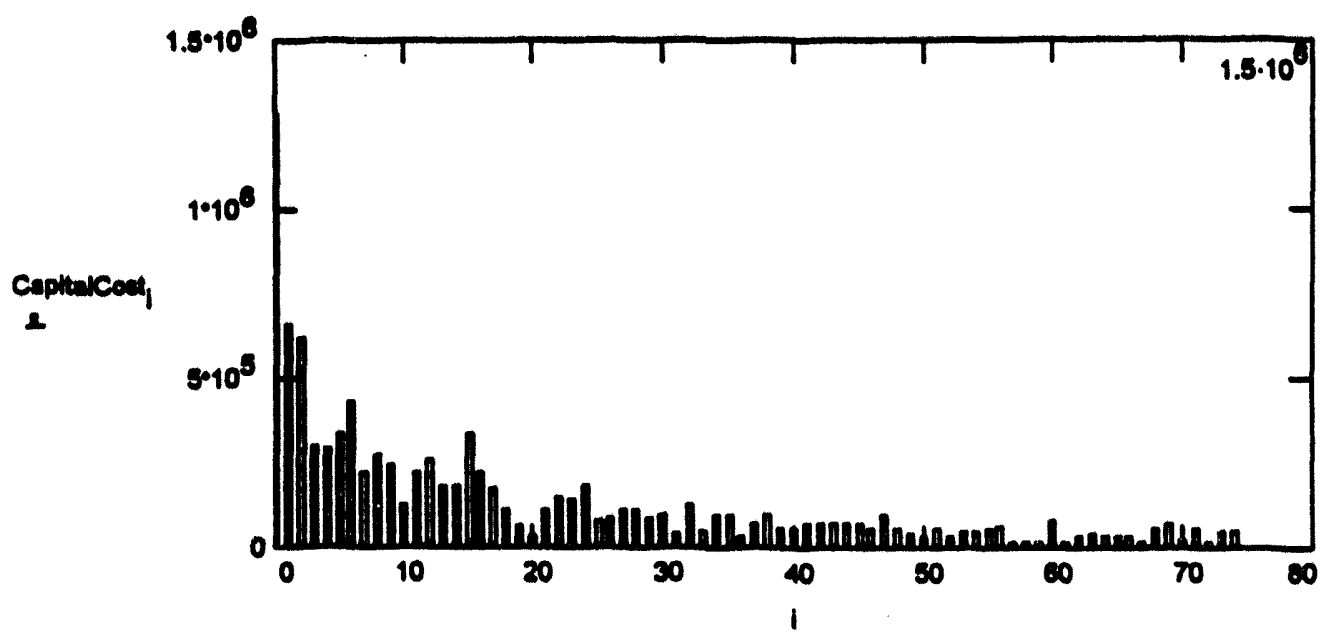

\section{SUM CAPITAL COSTS BY SIZT OF AREAS}

To sum couts for the larzeut 9 metropolttan area, (pop > $4 \mathrm{M})$ we sum from 0 to 8;

$$
\text { LargeAreaCost : }=\sum_{i} \text { CapitalCost },(i<9)
$$

LargeAreaCost $=4.4 \cdot B$

Simllarly,

$$
\begin{aligned}
& \text { MediumLargeAreaCost : }=\sum_{1} \text { CapitalCost }_{1} \cdot(i>8) \cdot(i<22) \\
& \text { MediumLargeAreaCost }=2.24 \cdot B
\end{aligned}
$$

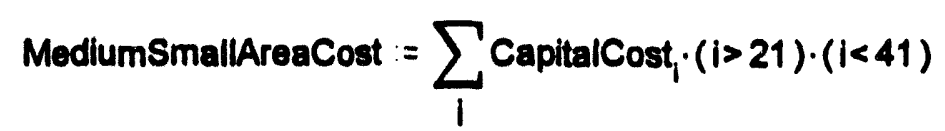

$$
\begin{aligned}
& \text { MediumSmallAreaCost }=1.75 \cdot B \\
& \text { SmallAreaCost }=\sum_{i} \text { CapitalCost }, \cdot(1>40) \\
& \text { SmallAreaCost }=1.56 \cdot \mathrm{B}
\end{aligned}
$$


SUM CAPITAL COSTS BY ELEMENT OF COST

ElementCapitalCost $_{1}=\sum_{1}$ ElementCost $_{(1, J)}$

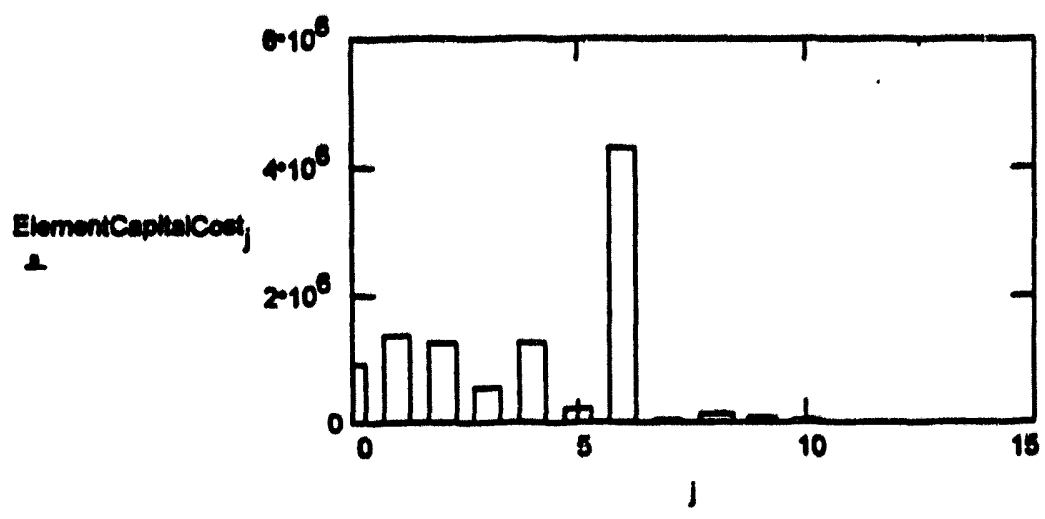

For reference, remember that

dement $0 \mathrm{~b}$ planning and enginearing cost

element 1 is senseor cost,

element 2 is signal coot,

element 3 is variablo-measage sign cost,

element 4 is bescon cost,

element 5 is radio cost,

element 6 is communication 'eabling' cont,

element 7 is computer hardware original cost,

element 8 is computer software ortyinal cost,

element 9 is staft coot,

element $10 \mathrm{~b}$ cost of providing bullding apace, and

element 11 is 'other' - to be defined - cost.

\section{SUM CAPITAL COSTS FOR ALL METROPOUTTAN AREAS}

To eatimate the grand total capital coat for all areas, we simply sum the eatimated costs for every area.

$$
\text { GrandTotalCapital : }=\sum_{1} \text { CapitalCost, }
$$

With the stated essumptions, the grand total b

$$
\text { GrandTotalCapival }=8.95 \cdot B
$$


To estimate costs for operations and muintenance, we take $10 \%$ of the capital cout for an area for maintenance - and add the projected other recurring costs (for ezample, aningy and training).

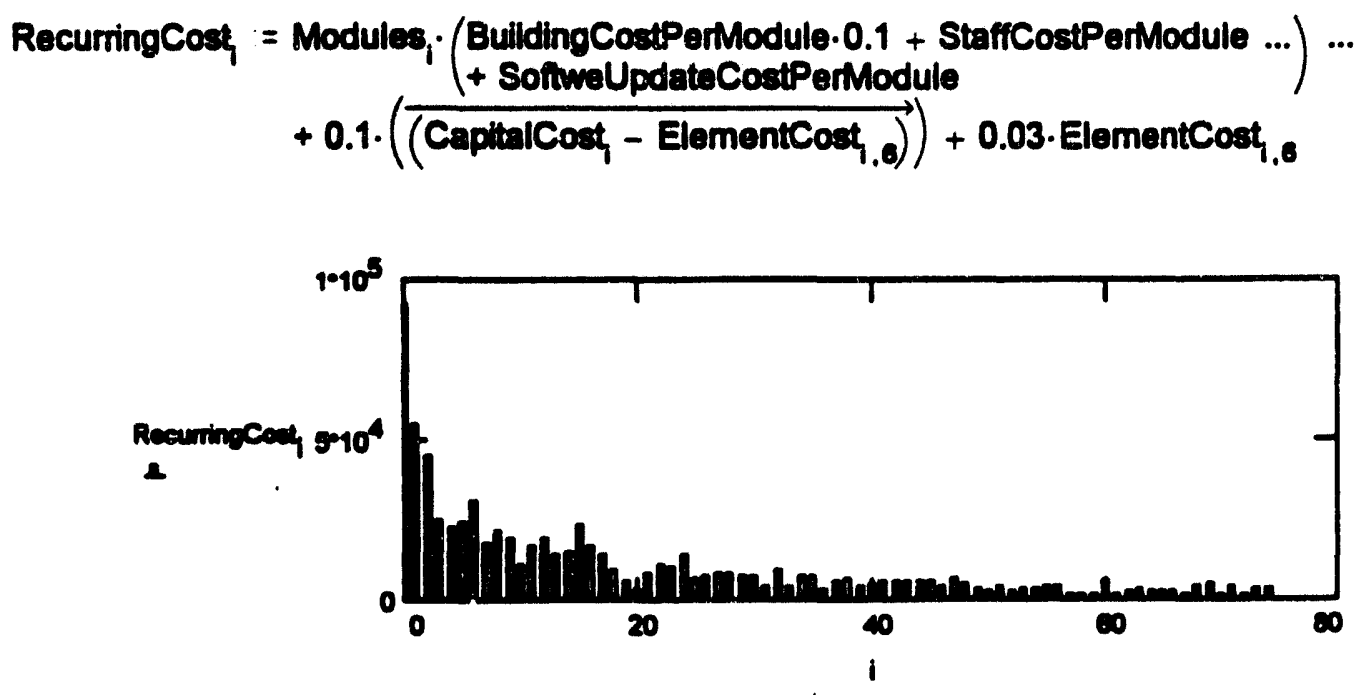

\section{SUM RECURRING COSTS BY STTE OF AREA}

$$
\begin{aligned}
\text { LargeAreaRecumingCost }:=\sum_{i} \text { RecumingCost } \cdot(i<9) \\
\text { MediumLargeAreaRecurringCost : }=\sum_{i} \text { RecurringCost } \cdot(i>8) \cdot(i<22) \\
\qquad \text { MediumLargeAreaRecurringCost }=176.33 \cdot M \\
\text { MediumSmallAreaRecurringCost : }=\sum_{i} \text { RecurringCost } \cdot(i>21) \cdot(i<41)
\end{aligned}
$$

$$
\begin{gathered}
\text { SmallAreaRecurringCost : }=\sum_{i} \text { RecurringCost } \cdot(i>40) \\
\text { SmallAreaRecurringCost }=125.05 \cdot M
\end{gathered}
$$

TotalRecumingCost : $=\sum_{i}$ RecumingCost

$$
\text { TotalRecurringCost }=0.77 \cdot B
$$


To eatimate the diatribution of ATMS implementation costs over the next five yearn, we begin by assuming spending profiles for projects. We sasume that large projects take loager than amall projects, but that the shapes of the spending curves are similar. We further assume that large areas are fartber along in the ATMS process, and bence can begin sooner. For convenience, we store the proflles in a separate ASCII tile called TIMEDIST.PRN', where each row of the file contains the fraction of total project cost that is spent during a particular 3-month interval and the 20 rows therefore represent spending profles for five years. Again, we access the file with a 'RSADPRN' statement and store the data in a temporary array.

$$
\text { TimeCoeffs := READPRN (imedist pm) }
$$

$$
\begin{aligned}
& \text { Index the data by quarters, where qtr := } 0 . .19 \\
& \text { and index the area ches by } k \text {, where } k:=0 . .3 \quad \text { ( } 0^{\prime} \text { b largeat) } \\
& \text { Recall that } K=1 \quad M=1 \cdot 10^{3} \cdot K \quad B=1 \cdot 10^{3} \cdot M \\
& \text { LargeAreaCost }=4.4 \cdot B \\
& \text { MediumLargeAreaCost }=2.24 \cdot B \\
& \text { MediumSmallAreaCost }=1.75 \cdot B \\
& \text { SmallAreaCost }=1.56 \cdot B \\
& \text { QtrCost }_{\phi, 1}:=\text { TimeCoeffs }_{\phi, 1} \cdot \text { MediumLargeAreaCost } \\
& \text { QurCost }_{\phi, 2}:=\text { TimeCoeffs }{ }_{\phi, 2} \cdot \text { MediumSmallAreaCost } \\
& \text { QtrCost }_{4 \pi, 3}:=\text { TimeCoeffs }_{4 r, 3} . \text { SmallAreaCost }
\end{aligned}
$$

For example,

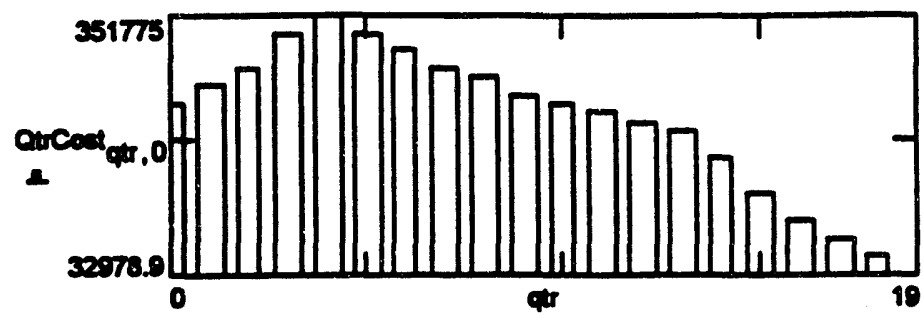

To get total implementation cost by year, we sum over the area costs and then sum the quarters in groups of foar.

$$
\text { QtrSum }_{\phi}:=\sum_{k} \text { QtrCost }_{\phi t, k}
$$




$$
\begin{aligned}
& \text { AnnualCost }_{0}:=\sum_{q t r} \text { QtrSum }_{q t r} \cdot(q t r<4) \\
& \text { AnnualCost }=1.12 \cdot B \\
& \text { AnnualCost } 1:=\sum_{q t r} \text { QtrSum }_{\text {qut }} \cdot(\text { qtr }>3) \cdot(\text { qtr }<8)
\end{aligned}
$$

$$
\text { AnnualCost }_{1}=2.35 \cdot \mathrm{B}
$$

$$
\text { AnnualCost }_{2}:=\sum_{q t} \text { QtrSum }_{\text {qu }} \cdot(\text { qtr> } 7) \cdot(q t r<12)
$$

$$
\text { AnnualCost }_{2}=3.08 \cdot \mathrm{B}
$$

$$
\text { AnnualCost }:=\sum_{\text {qtr }} \text { QtrSum }_{\phi r} \cdot(\text { qtro } 11) \cdot(\text { qtr }<16)
$$

AnnualCost $_{3}=2.36 \cdot \mathrm{B}$

$$
\text { AnnualCost }_{4}:=\sum_{\text {qtr }} \text { QtrSum }_{\text {qr }} \cdot(\text { qtr }>15)
$$

$$
\text { AnnualCost }_{4}=1.03 \cdot B
$$

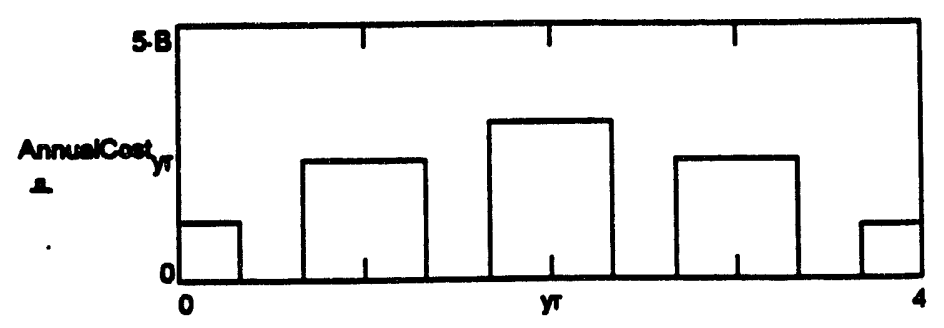

As a check,

$$
\begin{aligned}
\text { SumOfCost : }=\sum_{y r} \text { AnnualCost }_{y r} \quad \text { SumOfCost } & =9.95 \cdot B \\
\text { GrandTotalCapital } & =9.95 \cdot B
\end{aligned}
$$


We will begin by defining the individual elements of cost of implementing ATMS in the largeat 75 metropolitan areas, the characteriatics of the areas themselves, and the dependencies of the elements on size, milies, or some other characteristic.

Estimated cost for each area will then be computed by summing the element costs for that area. Estimated total cost of implementation will then be computed by aumming the area costs.

Data about the Individual areas will be stored and maintained external to this model and read into the computation when the model be executed. Some values are experts' eatimates, and derived values are assumed explicitly in the model. In this report, all avallable metropolitan area data are utilized. The data will be stored outelde the model

\section{INDEX DEFINIIIONS}

Let the metropolitan areas be indesed by 'I', where

$$
\begin{aligned}
& i:=0.74 \text { and the areas are those identified in the } \\
& 1990 \text { census (e.g., New York, the largest, is area 0), }
\end{aligned}
$$

and let the cost elements by indexed by ' $\mathrm{j}$ ', where

$$
j:=0 . .11
$$

element 0 is planning and engineering cost,

element 1 is seasor cost, element 2 is aignal cost, element 3 is variable-measage sign cost, element 4 b beacon cont (which will be 0 in rome architecturea), element $5 \mathrm{~b}$ radio cost (which will be $0 \mathrm{ln}$ some architectures), element 6 b communication 'cabling' cost, element 7 is computer hardware original coat, element 8 b computer software original costh, element 9 is stafi cost, element 10 is cost of providing bullding apace, and element 11 is 'other' - to be defined - cost.

Costs are stated as per-mile or other appropriate unit costs, and then multiplied by the appropriate factor to calculate element cost for a particular area. 


\section{READ IN AREA CHARACTERISTIC DATA}

Assume that some of the characteristics of the metropolitan areas are stored in the rows of an ASCD file (named "ascidat.pm"), ordered by size rank, with each row of the file containing the area's population, number of freeway miles, number of arterial milles, number of signalized intersections, and adjustment factors for geographic location and curreat status of advanced trafilc management planning . or implementation. We access the flle with 'READ' statements. For programming convenience, we'll define a data matrix for temporary storage, read into th, and pick out the individual characteriatic data for further use.

Index the characteriatica by ' $k$ ', where

$$
k:=0 . .5
$$

$$
\begin{aligned}
& \text { Then data := READPRN (asciidat) } \\
& \text { Pop }_{1}:=\text { data }_{1,0} \\
& \text { Modules, }:=\operatorname{ceil}\left(\frac{P_{0 p_{1}}}{10^{3}}\right) \quad \text { Number of TMC modules } \\
& \text { Fuymil }_{1}=\text { data }_{1,1} \quad \text { Number of freeway miles } \\
& \text { MinArtM }_{1}:=\text { data }_{1.2} \quad \text { Number of arterial miles } \\
& \text { AugArtMi }:=\text { data }_{(1,2)} \cdot 5 \quad \text { Number of (augmented) arterial miles } \\
& \text { Aug := } 1 \text { 'Aug' lets us consider the two caseson. } \\
& \text { TotMi : if }(\text { Aug }>0, \text { FwyMi }+ \text { AugArtMi, FwYMi }+ \text { MinArtMi }) \\
& \text { Intrsect, }:=\text { If }\left(\text { Aug }_{1}>0, \text { data }_{1,3} \cdot 5, \text { data }_{1,3}\right)
\end{aligned}
$$

Number of intersections controlled
GeoFactor $_{1}:=$ data $_{1.4} \quad$ Factor to adjust for differing conatruction costs by climate and difrering internection density

Statusfactor $_{i}=$ data $_{1,5} \quad$ Factor to adjust for planning and implementation already existing 


\section{DEFINE UNIT COSTS FOR ARCHITECTURE 2}

Costs will be given in $\mathrm{K}$ (thousands), $\mathrm{M}$ (millions), or $\mathrm{B}$ (billions)

$$
\text { where } K=1 \quad M=1000 \cdot K \text { and } B=1000 \cdot M
$$

SignalCostPerintrsect : $=40 \cdot \mathrm{K}$

$$
\text { Fr old : }=0.5
$$

BeaconCostPerMile : $=\mathbf{5 0} \cdot \mathbf{K}$

CommCostPerMile : $=150 \cdot \mathrm{K}$

SoftwelnstanceCostPerMile : $=2 \cdot \mathrm{K}$

BuildingCostPerModule $:=200 \cdot K$

OtherCostPervile : $=0$

(will be added as needed)
SensorCostPerMile $=\mathbf{5 0} \cdot \mathrm{K}$

VMSCostPerMile $=40 \cdot \mathrm{K}$

RadioCostPerMile $=15 \cdot K$

CompHdweCostPerModule : $=125 \cdot \mathrm{K}$

StaffCostPerModule $:=400 \cdot K$

SoftweOrigCostPerModule : $=300 \cdot \mathrm{K}$

SoftweUpdateCostPerModule $=15 \cdot \mathrm{K}$

\section{CALCULATE COST ELEMENTS FOR EACH AREA}

The elements of cost, as libted above, can then be calculated for each specinc area.

$$
\begin{aligned}
& \text { ElementCost }_{(i, 0)}:=0 \quad \text { (will add in } \$ s \text { 10\% after calculation of capital cost) } \\
& \text { ElementCost }_{1,1}:=\text { SensorCostPerMile.StatusFactor } \cdot \text {-GeoFactor }, \text { TotMi }_{i} \cdot K \\
& \text { ElementCost, } 1,2=\text { SignalCostPerintreect. }\left(\overrightarrow{\left(\text { Intreect, } \cdot \mathrm{Fr}_{\text {old }}+\mathrm{FwyM}_{1}\right)}\right) \cdot \mathrm{K} \\
& \text { ElementCost } 1,3 \text { : = VMSCostPerMile.FwyMi } \cdot \text { GeoFactor } \cdot \text { StatusFactor } \cdot K \\
& \text { ElementCost }_{1,4}:=\text { BeaconCostPerMile }_{[}\left[\left(\text {FwyMi }_{i}+\text { MinArtMi }\right) \cdot K\right] \\
& \text { ElementCost }_{1,5}:=\text { RadioCostPerMile. FmyMi } \cdot K \\
& \text { ElementCost }_{1,6}:=\text { CommCostPerMile.TotMi, GeoFactor }_{1} \cdot K \\
& \text { ElementCost } 1,7:=\text { CompHoweCostPerModule } \cdot \text { Modules }_{1} \cdot K \\
& \text { ElementCost }_{1,8}:={\text { SoftwelnstanceCostPerMile } \cdot \text { TotMi }_{1} \cdot K}+\left(\text { SoftweOrigCostPerModule } \cdot \text { Modules }_{1}\right. \text { ) } \\
& \text { ElementCost } 1,0:=\text { StaffCostPerModule } \cdot \text { Modules, } \cdot K \\
& \text { ElementCost }_{1,10}:=\text { BulldingCostPerModule } \cdot \text { Modules }_{i} \cdot K \\
& \text { ElementCost }_{(1,11)}:=\text { OtherCostPerMile.K }
\end{aligned}
$$




\section{SUM CAPITAL COSTS FOR THE INDIVDUAL METROPOLTTAN AREAS}

With elemeats of cost and characteristies of the Individual areas defloed, total eotimated cost for each area can be caleulated by summing over the elements of cost.

InstalledCost $:=\sum_{J}$ ElementCost $_{1, j}(j<9)$

CapitalCost $:=$ InstalledCost, $1.1 \quad$ ElementCost $_{(1,0)}:=$ InstalledCost $\cdot 1$

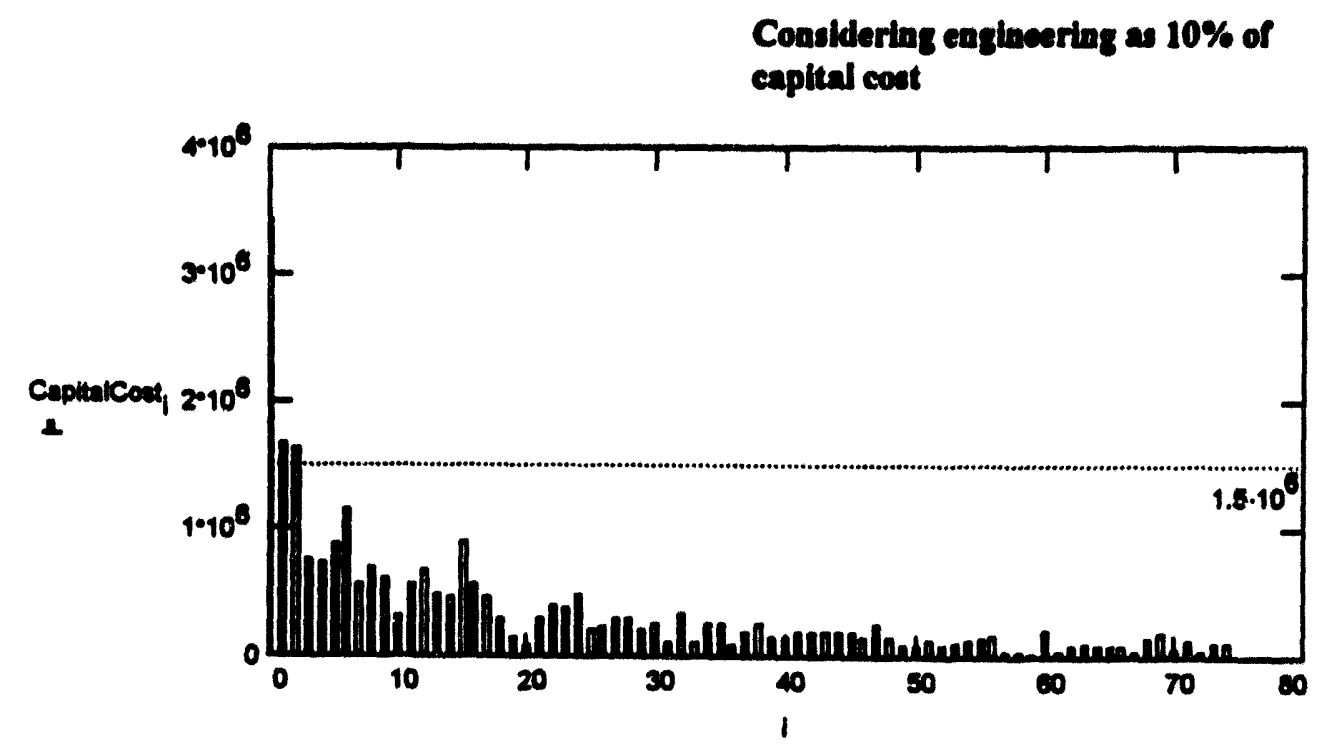

\section{SUM CAPITAL COSTS BY SIZE OF AREAS}

To sum costs for the largeat 9 metropolitan areas, (pop > 4M) we sum from 0 to 8;

$$
\text { LargeAreaCost : }=\sum_{i} \text { CapitalCost }_{i}(i<\theta)
$$

LargeAreaCost $=11.48 \cdot B$

Similarly,

$$
\begin{aligned}
\text { MediumLargeAreaCost }= & \sum_{i} \text { CapitalCost }, \cdot(1>8) \cdot(i<22)_{\text {MediumLargeAreaCost }=5.79 \cdot B}
\end{aligned}
$$$$
\text { MediumSmallAreaCost }=\sum_{i} \text { CapitalCost }_{i} \cdot(i>21) \cdot(i<41)
$$$$
\text { ModiumSmallAreaCost }=4.55 \cdot B
$$

SmallAreaCost : $=\sum_{i}$ CapitalCost $_{j} \cdot(i>40)$

SmallAreaCost $=4.03 \cdot B$ 


$$
\text { ElementCapitalCost }_{1}=\sum_{1} \text { ElomentCost }_{(1, j)}
$$

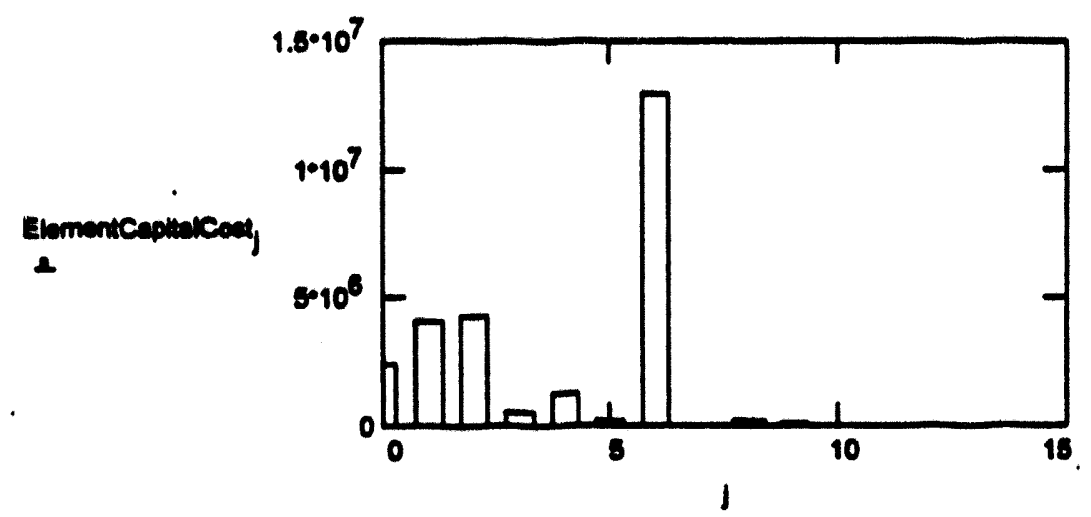

For reference, remember that

element 0 is planuing and engincering cost

element 1 is censor cost, element 2 is signal cost, element 3 is variablo-measage aign cont, clement 4 is bescon cost, element 5 is radio cont, element 6 is communication 'cabling' cost, element 7 b computer hardware original cost, element 8 is cosputer software origlinal cost, element 9 is stafi cost, element 10 b cont of providing bullding apace, and element 11 is 'other' - to be.defined - coet.

\section{SUM CAPITAL COSTS FOR ALL METROPOUTTAN AREAS}

To eatimate the grand total capttal cost for all areas, we simply sum the eatimated coats for every area.

$$
\text { GrandTotalCapital }=\sum_{1} \text { CapitalCost }
$$

With the stated assumptions, the grand total is

GrandTotalCapltal $=25.84 \cdot B$ 


\section{SUM RECURRING COSTS FOR INDIVDUAL METROPOLTTAN AREAS}

To entimate coots for operations and maintenance, we take $10 \%$ of the capital cost for an arva for maintenance - and add the projected other rocurring conts (for exampla, aslany and training).

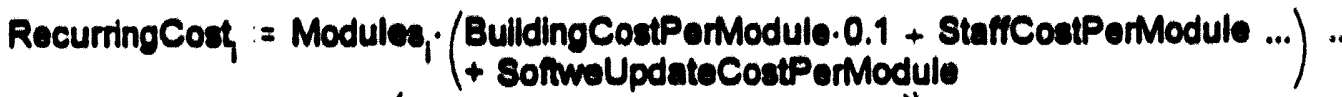

$+0.1 \cdot(\overrightarrow{(\text { CapitalCost }- \text { ElementCost. }}))+0.03 \cdot$ ElementCost $_{1.0}$

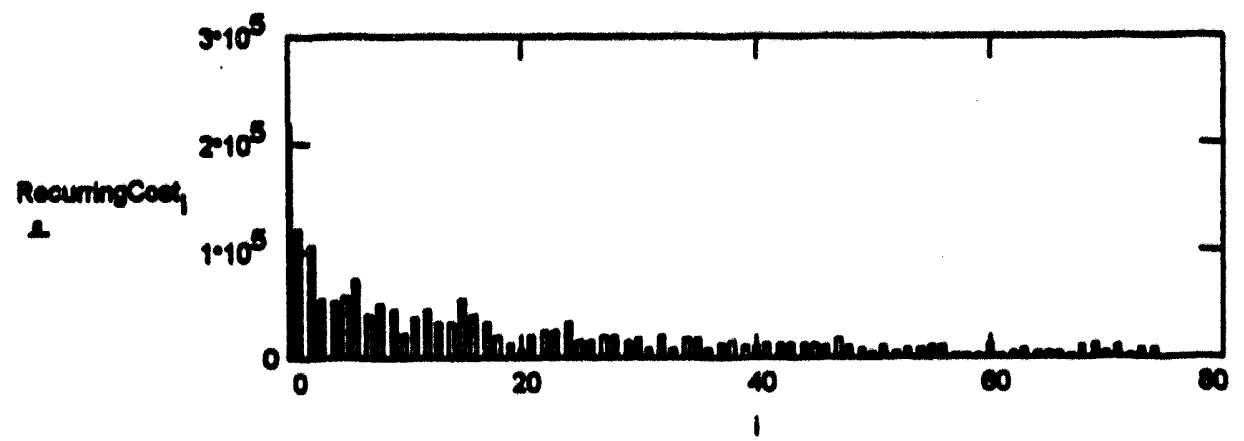

SUM RECURRING COSTS BY SIEE OF AREA

LargeAreaRecurringCost : $=\sum_{i}$ RecurringCost $\cdot(i<\theta)$

LargeAreaRecurringCost $=768.38 \cdot M$

MediumLargeAreaRecurringCost : $=\sum_{1}$ RecurringCost $\cdot(i>8) \cdot(i<22)$

MediumLargeAreaRecurringCost $=400.47 \cdot M$

MediumSmallAreaRecurringCost := $\sum_{i} R_{\text {ecurringCost }} \cdot(1>21) \cdot(1<41)$

MediumSmallAreaRecurringCost $=312.77 \cdot M$

SmallAreaRecurringCost $=\sum_{i}$ RecurringCost. $\cdot(i>40)$

SmallAreaRecurringCost $=280.64 \cdot M$

TotalRecurringCost : $=\sum_{1}$ RecurringCost

TotalRecurringCost $=1.76 \cdot B$

Arch 2 Augmented Network 


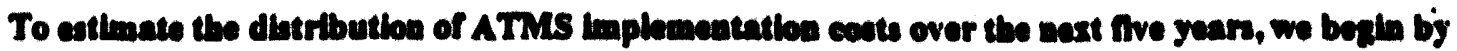

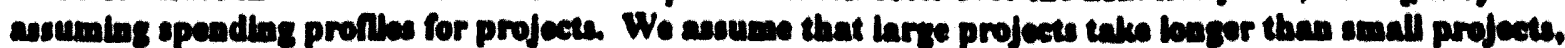
but that the shapes of the apeadiag curves are abmillar. We further sesume that large areas are furthor.

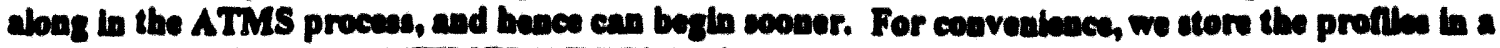

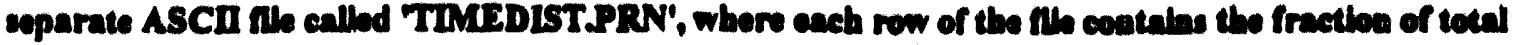

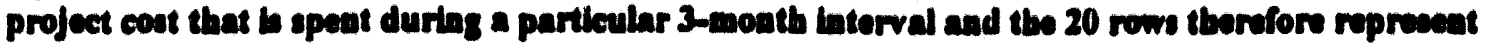
speadlos profles for five ycias. Agaln, we sceess the file with a 'READPRN' statement and store the data lo a temporary array.

$$
\text { Timocoofis : = READPRN (tmodint pm) }
$$

$$
\begin{aligned}
& \text { Indes the data by quarters, where } \\
& \text { qut }:=0 . .10 \\
& \text { and bidex the area abeo by bo, where } \\
& k=0 . .3 \quad\left(0^{\prime} b\right. \text { langent) } \\
& \text { Recall that } \quad K=1 \quad M=1 \cdot 10^{3} \cdot K \quad B=1 \cdot 10^{3} \cdot M \\
& \text { and } \\
& \text { LargeAreaCoet }=11.48 \cdot B \\
& \text { ModiumLargeAruaCost }=5.79 \cdot 8 \\
& \text { Mediumsmallarencoet }=4.55 \cdot B \\
& \text { Smallareacost }=4.03 \cdot B \\
& \text { QtrCost }_{\phi, 1}:=\text { TimoCoefis }_{\phi, 1} \cdot \text { MediumLargeArescost }
\end{aligned}
$$

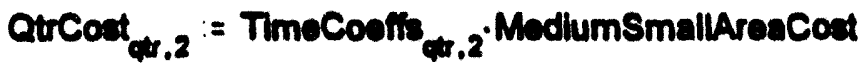

$$
\begin{aligned}
& \text { QurCost }_{\phi, 3}:=\text { TimoCoofis }{ }_{\phi r, 3} . \text { SmallAreaCost }
\end{aligned}
$$

For example,

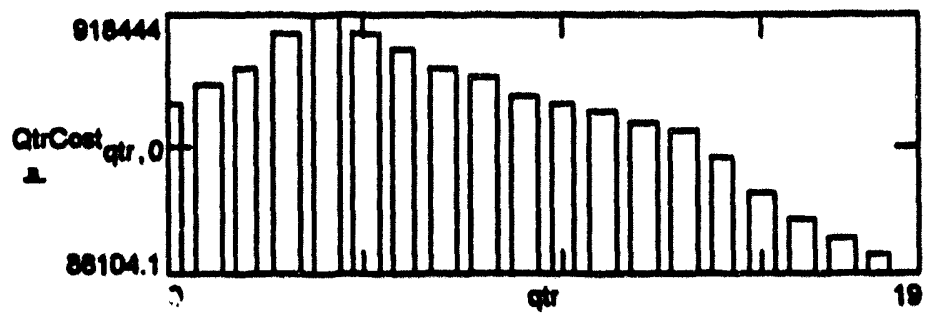

To get total implementation cost by year, we sum over the area coats and then sum the quarters in Eroups of four.

$$
\text { Qtrsum }_{\text {at }}:=\sum_{k} \text { QtrCost }_{\phi \pi, k}
$$




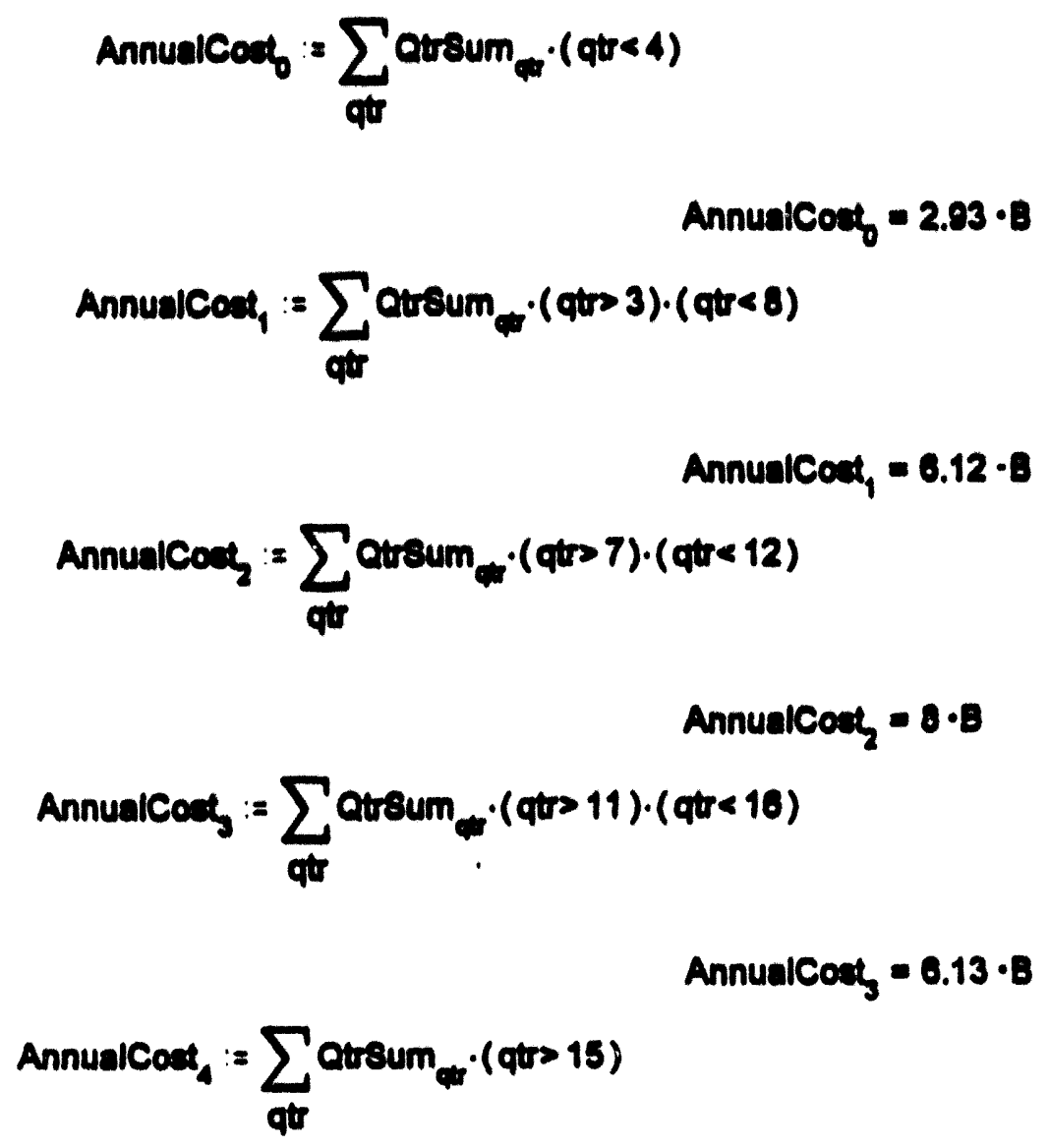

Annualcost $_{4}=2.67 \cdot B$

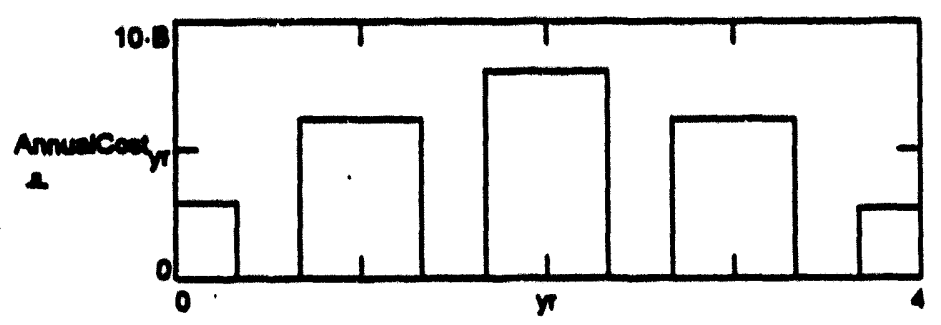

As a chock,

$$
\begin{aligned}
\text { SumOrCoat }:=\sum_{y r} \text { AnnualCost }_{r r} \quad \text { SumOrCost } & =25.84 \cdot B \\
\text { GrandTotalCapltal } & =25.84 \cdot B
\end{aligned}
$$




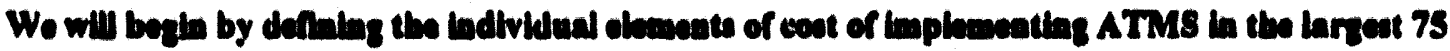

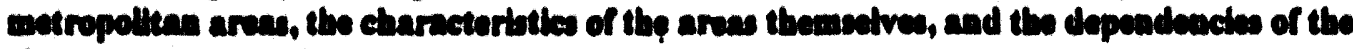

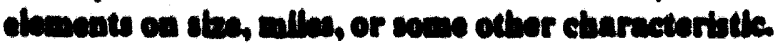

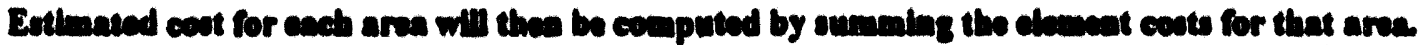

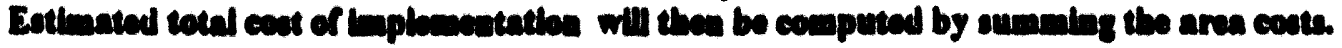

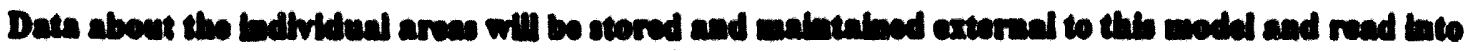

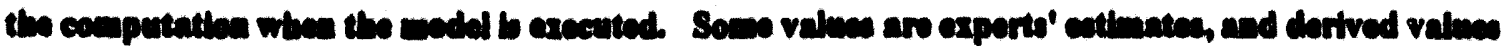

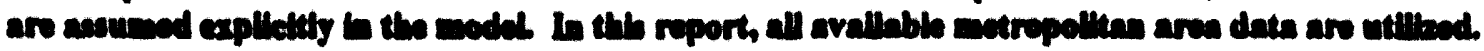

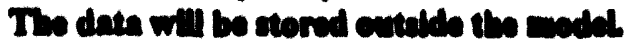

\section{INDEX DENTIMONS}

Let the motropeltina areas bo bedered by 'I', whore

$$
1=0.74 \text { and the arvas are thoce bloatined b the }
$$

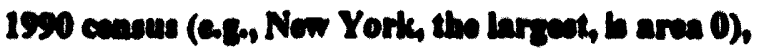

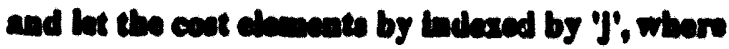

$$
J:=0 . .11
$$

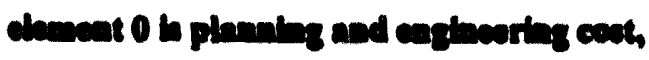

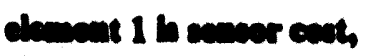

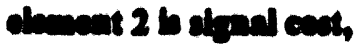

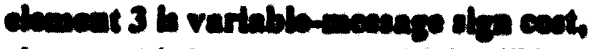

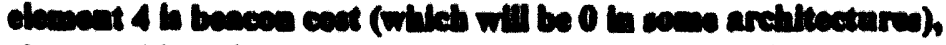

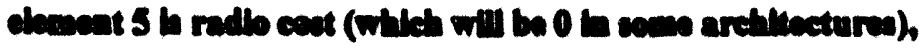

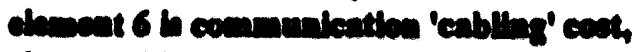
cloment 7 b compector bantwan ortejunl cact, clement 8 b computer seliware ortafidel eceh clanenat $9 \mathrm{~b}$ ataf coeth

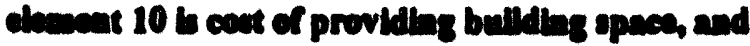
dameat 11 b 'othar' - to be dofined - ceat.

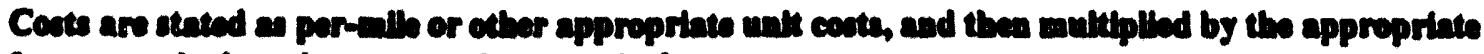
fector to calculate olemente cout for a particalar area. 


\section{READ IN AREA CHARACTERISTIC DATA}

Assume that some of the charactertaties of the metropoltten areas are stored io the rows of an ASCI The (asened "acetidatprn"), ordared by stee rank, with each row of the file containing the arra's

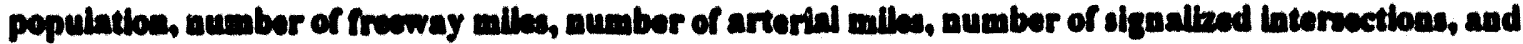

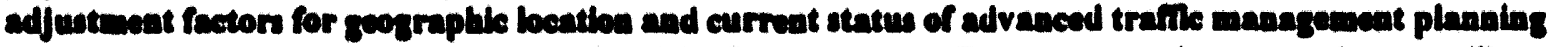

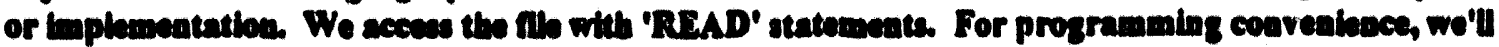

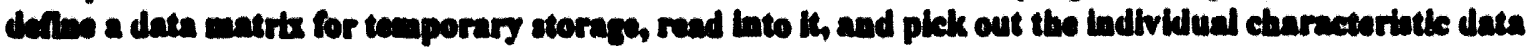
Por turtbor une

Pop : $=\operatorname{dath}_{1,0}$ Modulea, $:=\operatorname{cell}\left(\frac{\text { Pop }}{10^{3}}\right)$ Finsim : $:=$ datan $_{1,1}$

Minarem := data,

Augarmy : $=\operatorname{dath}(1,2) \cdot 5$
Population, in thousands

Numbor of TMC modules

Number of fresway milles

Number of arterial milles

Number of (angmented) artarial milles

Awg : = 0 'Ang' beto wo conclder the two cascems

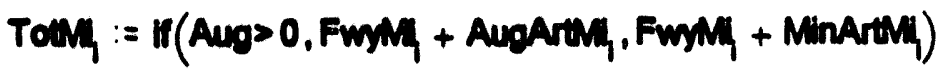

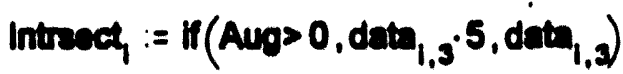

Number of intersections controlled

Geofactor, $:=$ dath $_{1,4}$

Stuturafactor, : $\operatorname{data}_{1,5}$
Factor to adjuet for ditiortas

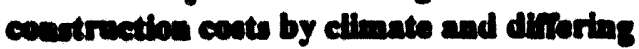
intersection deanity

Factor to adjuet for plamiles and Inplomentation already exintios 
DEFINE UNIT COSTS FOR ARCHTTECTURS 3

Coste will be given in $\mathbf{K}$ (thousands), $\mathbf{M}$ (millions), or B (billioas)

$$
\text { where } K=1 \quad M=1000 \cdot K \text { and } B=1000 \cdot M
$$

SignalCostPerintreect $=40 \cdot \mathrm{K}$

$F_{\text {old }}=0.5$

Beaconcostperivile : $=0 . K$

Commcostperivile : $=150 \cdot K$

SoltwolnatinceCoutPerkile : $=2 \cdot K$

BulldingCoutPerModule : $=200 \cdot K$

Othercoetporille : : 0

(will be addad as neodod)
SensorCostPerMile $=\mathbf{5 0} \cdot \mathrm{K}$

VMSCostPorMile $=40 \cdot \mathrm{K}$

RadioCostPorMile $=15 \cdot \mathrm{K}$

ComptidweCostPerModule $=175 \cdot \mathrm{K}$

StaffCoetPerModule : $=400 \cdot \mathrm{K}$

SoltwoOrigCostPerModule : $=350 \cdot \mathrm{K}$

SolmwoUpdateCostPerModule : $=15 \cdot \mathrm{K}$

\section{CALCULATE COST WLMMENTS FOR EACH ARRA}

The elements of cont, as libted above, can theo be calculated for each apecinc aree.

$$
\begin{aligned}
& \text { Elementcoet }_{(1,0)}:=0 \quad \text { (will add in w } 10 \% \text { sfior calculatibe of eapital coet) } \\
& \text { ElementCost } 1,1 \text { = SensorCoatPerMile. StatuaFactor, GeoFactor, Tothi, } K
\end{aligned}
$$

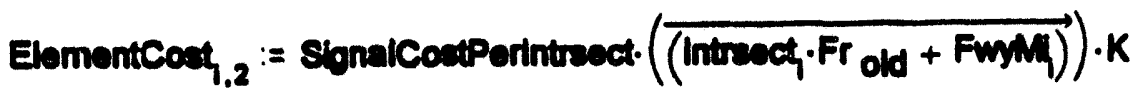

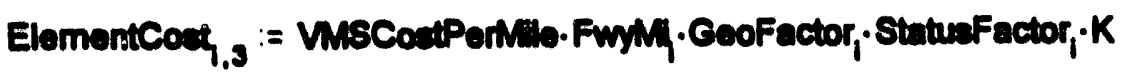

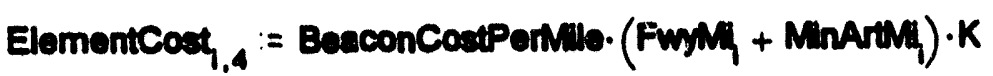

$$
\begin{aligned}
& \text { ElementCoet, } 1,5 \text { RadioCostPerMilo. Funim. } K \\
& \text { ElomentCost, : = CommCodPerMib.TotM. Geofactor, } K \\
& \text { ElementCost }, 7:=\text { ComphtwweCodPerModule } \cdot \text { Modules, } \cdot K
\end{aligned}
$$

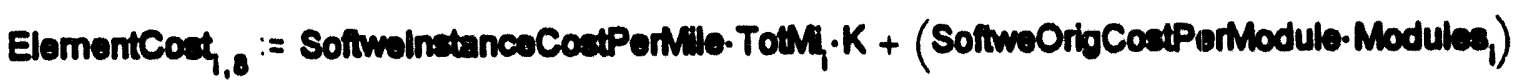

$$
\begin{aligned}
& \text { ElementCost, : = StancoetPerModulo.Modulea, } \cdot K \\
& \text { ElementCost, } 10:=\text { BulldingCoetPerModulo.Modules, } \cdot K \\
& \text { ElementCost }_{(1,11)}:=\text { OtherCostPerMilo.K }
\end{aligned}
$$


With elements of cost and characterbtics of the individual areas defined, total estimated cost for each area can be calculated by sumbing over the elementu of cost.

$$
\begin{aligned}
& \text { InstalledCost, }:=\sum_{j} \text { ElementCost }_{1, j}(j<9) \\
& \text { CapitalCost }:=\text { InstalledCost } \cdot 1.1 \quad \text { ElementCost }_{(i, 0)}=\text { InstalledCost } \cdot .1
\end{aligned}
$$

Conoidering engincering as $10 \%$ of capital coot

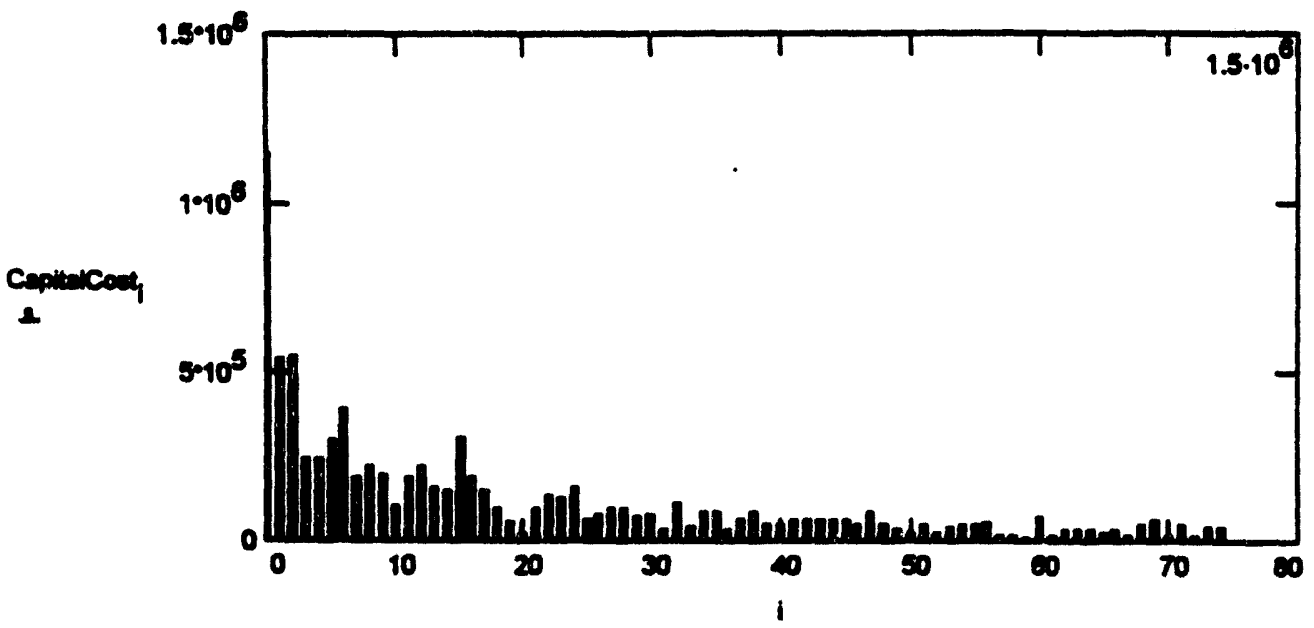

\section{SUM CAPITAL COSTS BY STER OF AREAS}

To sum coets for the largent 9 metropellina areas, (pop > 4M) we sum from 0 to 8;

$$
\text { LargeAreaCoet := } \sum_{i} \text { CapitalCost } \cdot(i<\theta)
$$

LargeAreaCost $=3.82 \cdot B$

Stmilarty,

$$
\begin{aligned}
& \text { MediumLargaAreaCost }:=\sum_{i} \text { CapitalCost } \cdot(i>8) \cdot(i<22) \\
& \text { MediumLargeAreaCost }=1.91 \cdot B \\
& \text { ModiumSmallAreaCost }:=\sum_{i} \text { CapitalCost } \cdot(i>21) \cdot(i<41) \\
& \text { SmallAreaCost }:=\sum_{i} \text { CapitalCost } \cdot(i>40) \\
& \text { SmallAreaCost }=1.34 \cdot B
\end{aligned}
$$


SUM CAPITAL COSTS BY ELEMENT OF COST

ElementCapitalCost $_{j}=\sum_{i}$ ElementCost $_{(1, j)}$

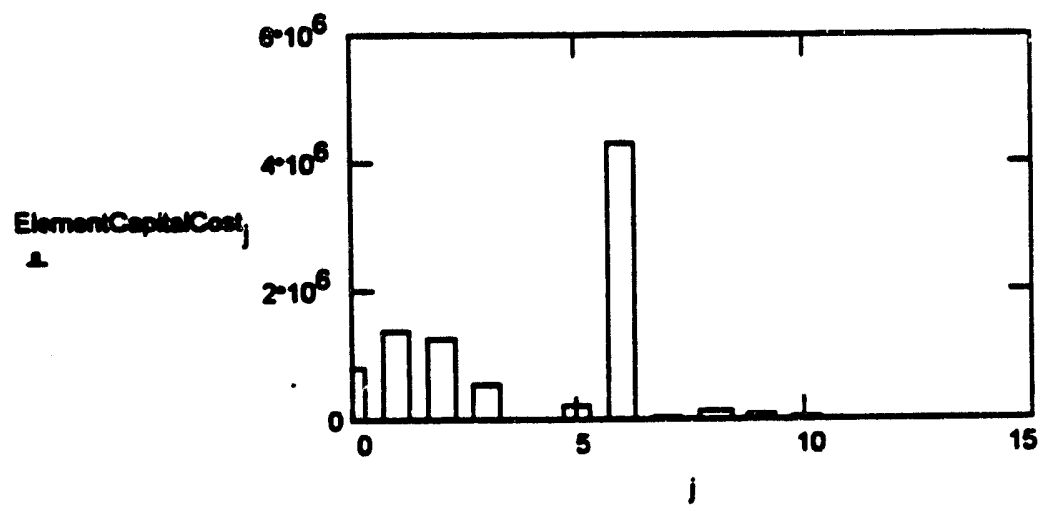

For reference, remember that

element 0 is planning and enginewring coat

element 1 b seavor costh

element 2 b olgmal ciath

element 3 b variabbo-measage oliza costh

element 4 is beacon cout,

element 5 is radio coat,

element 6 is comanunication 'cabling' coot,

element 7 b computer handware orighal cost,

clement 8 b computer ecfiware orighal cooth

clement 9 is atefir coeth

clement $10 \mathrm{~b}$ cont of providing bullding opace, and

element 11 is 'other' - to be deined - coet.

\section{SUM CAPITAL COSTS FOR ALL METROPOLTAN AREAS}

To estimate the grand total capital cont for all arean, we simply sum the estimated costs for every area.

$$
\text { GrandTotalCapital : }=\sum_{i} \text { CapitalCost }
$$

Whth the stated assumptions, the grand total is

GrandTotalCapital $=\mathbf{8 . 5 9} \cdot \mathrm{B}$ 


\section{SUM RECURRING COSTS FOR INDIVDUAL METROPOLTTAN AREAS}

To estimate costs for operations and maintenance, we take $10 \%$ of the capital coat for an area for maintenance - and add the projected other recurring costs (for example, salary and uraining).

RecurringCost $:=$ Modules, $;\left(\begin{array}{l}\text { BuildingCostPerModule } \cdot 0.1+\text { StaffCostPerModule } . . .) \\ + \text { SoftweUpdateCostPerModule }\end{array}\right)$ $\left.+0.1 \cdot\left(\overrightarrow{\left(\text { CapitalCost }_{1} \text { - ElementCost }, 6\right.}\right)\right)+0.03 \cdot$ ElementCost $_{1,6}$

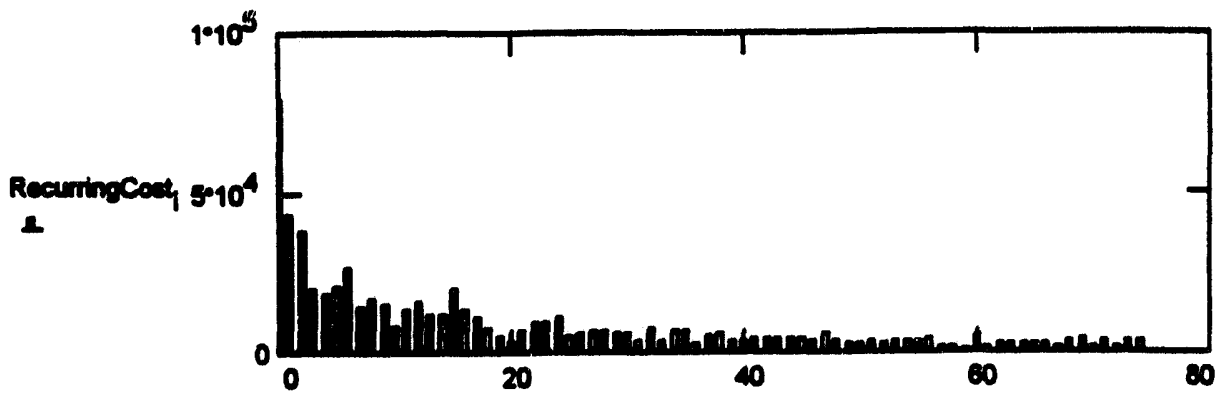

\section{SUM RECURRING COSTS BY SIZE OF AREA}

$$
\begin{aligned}
& \text { LargeAreaRecurringCost : }=\sum_{i} \text { RecurringCost } \cdot(i<\theta) \\
& \text { LargeAreaRecurringCost }=276.58 \cdot M \\
& \text { MediumLargeAreaRecurringCost : }=\sum_{i} \text { RecurringCost } \cdot(i>8) \cdot(i<22) \\
& \text { MediumLargeAreaRecurringCost }=143.61 \cdot M \\
& \text { MediumSmallAreaRecurringCost := } \sum_{i} \text { RecurringCost, }(i>21) \cdot(i<41) \\
& \text { ModiumSmallAreaRecurringCost }=114.82 \cdot M \\
& \text { SmallAreaRecurringCost : }=\sum_{i} \text { RecurringCost } \cdot(i>40) \\
& \text { SmallAreaRecurringCost }=102.98 \cdot \mathrm{M} \\
& \text { TotalRecurringCost : }=\sum_{1} \text { RecumingCost } \\
& \text { TotalRecurringCost }=0.64 \cdot \mathrm{B}
\end{aligned}
$$


To estimate the diatribution of ATMS implementation conts over the next five years, we begta by assuming speading proflice for projects. We assume that large projects iake longer than small projects. but that the shapes of the spendlog curves are similar. We further assume that large areas are farther along in the ATMS process, and hence can begin sooner. For convenience, we store the profiles in a separate ASCII nie called TIMEDIST.PRN', where each row of the file contains the fraction of total project cost that is apeat during a particular 3-month interval and the 20 rows therefore represeat spending profles for five years. Again, we access the file with a 'READPRN' statement and store the data in a temporary array.

$$
\text { TimeCoeffs : = READPRN (timedist prn) }
$$

$$
\begin{aligned}
& \text { Index the data by quarters, where } \\
& \text { qut }:=0 . .19 \\
& \text { and linder the area shea by } k_{4} \text { where } \\
& k:=0 . .3 \quad \text { ('0' b larteat) } \\
& \text { Recall that } \\
& K=1 \\
& M=1 \cdot 10^{3} \cdot K \\
& B=1 \cdot 10^{3} \cdot M \\
& \text { LargeAreaCost }=3.82 \cdot B
\end{aligned}
$$

MediumLargeAreaCost $=1.91 \cdot B$

MediumSmallAreaCost $=1.52 \cdot B$

SmallAreaCost $=1.34 \cdot B$

Then

$$
\begin{aligned}
& \text { QtrCost }_{\phi r, 0}=\text { TimeCoeffs } \text { qr, }, 0 \cdot \text { LargeArenCost }
\end{aligned}
$$

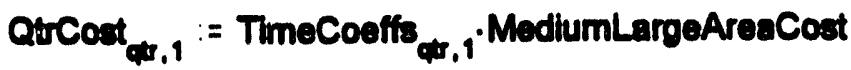

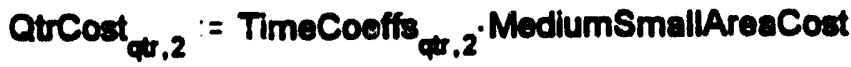

$$
\begin{aligned}
& \text { QurCost }_{q t, 3}:=\text { TimoCoeffs } \phi_{\phi, 3} \text { SmallAreaCost }
\end{aligned}
$$

For example,

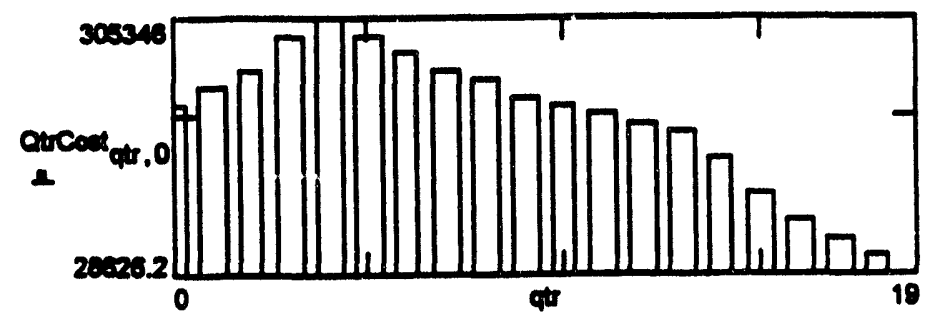

To get total implementation coat by year, we sum over the area conts and then sum the quarters in groups of four.

$$
\text { QtrSum }_{\phi}:=\sum_{k} \text { QtrCost }_{\phi, k}
$$




$$
\begin{aligned}
& \text { Let years be indezed by } \quad y r=0 . .4 \\
& \text { AnnualCost }_{0}=\sum_{q t r} \text { QtrSum }_{q t r} \cdot(q t r<4) \\
& \text { AnnualCost } 00.97 \cdot B \\
& \text { AnnualCost }_{1}:=\sum_{q t r} \operatorname{QtrSum}_{q t} \cdot(q t r>3) \cdot(q t r<8)
\end{aligned}
$$$$
\text { AnnualCost }_{1}=2.03 \cdot B
$$$$
\text { AnnualCost }:=\sum_{q t} \text { QtrSum }_{q \cdot} \cdot(q t r>7) \cdot(q t r<12)
$$

$$
\text { AnnualCost } 2=2.66 \cdot B
$$$$
\text { AnnualCost }_{3}:=\sum_{q t r} \operatorname{QtrSum}_{\text {qr }} \cdot(\text { qtr }>11) \cdot(\text { qtr }<16)
$$

AnnualCost $_{3}=2.04 \cdot B$

$$
\text { AnnualCost }_{4}:=\sum_{q t} \text { QtrSum }_{\phi} \cdot(q t r>15)
$$

$$
\text { AnnualCost }_{4}=0.89 \cdot B
$$

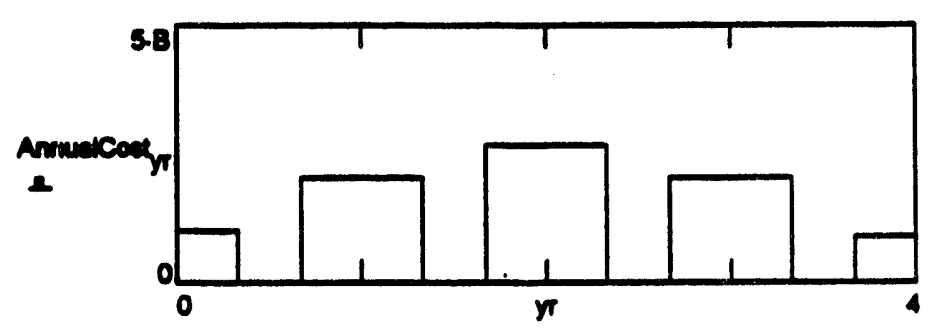

As a checkn,

$$
\begin{aligned}
\text { SumOfCost }:=\sum_{y r} \text { AnnualCost }_{y r} \quad \text { SumOfCost } & =8.59 \cdot B \\
\text { GrandTotalCapital } & =8.59 \cdot B
\end{aligned}
$$


We will begitn by defining the individual elemests of cont of implementing ATMS in the lareen 75 metropolltan areas, the charactoriatics of the areas themeelves, and the dopeadeaclas of the elements on sten, miles, or some othor chaructertitic.

Estimated cost for each area will then be computed by summing the element conts for that ares. Estimated total cost of buplamentation will then be computed by summing the area couts.

Data about the ladividual areas will be stored and malatalecd axtornal to thib model and road lato the computation when the model bs exceuted. Some values are experts' eatlenates, and dartred values are asoumed expllettly in the model. In this report, all avallable metropolttan area data are utlltbel. The data will be wtored eutalds the modal

\section{INDEX DEFINIMONS}

Let the metropolitan areas be lindezed by 'I', where

$$
\begin{aligned}
& 1:=0.74 \text { and the areas are those llentined to the } \\
& 1990 \text { cemsus (ess., Now Yorts the laryeoth bs area 0), }
\end{aligned}
$$

and let the cont elementu by molased by 'J', where

$$
J:=0 . .11
$$

clameat $0 \mathrm{~b}$ planning and eaghecertarg cooth

clement 1 b emeor ecoth

cloment 2 b olgand costh

clement 3 b vartablo-manage sign cooth

clement 4 is bescen cont (which will be 0 in some arehulueturew),

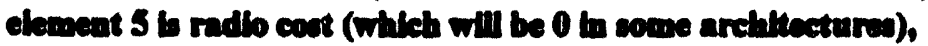

elemeat 6 b communication 'cabling' costh

eloment 7 b computor handware ortidnal cont,

elemeat 8 b computer eoftware ortigianl costh

element 9 b etafi costh

elemeat 10 b cost of providing bullding apacen and

clemeat 11 is 'other' - to be defined - cooth.

Coats are atated as per-milb or otwer appropriate unit costs, and thea multipliad by the approprtate factor to calculate element coot for a particular arae. 


\section{READ IN AREA CHARACTERISTIC DATA}

Assume that some of the characteristics of the metropolitan areas are stored in the rows of an ASCU The (named "asclidatprn"), ordered by she rank, with each row of the file containing the area's population, number of freway milee, number of artertal miles, number of alganalibed intersections, and adjustment factors for geographic location and current atatus of advanced tranic managemeat planning or implementation. We sccess the file with 'READ' statements. For programming coaveaibace, we'll define a data matrts for temporary storage, read into $\mathrm{H}$, and pick out the individual characteritic data for further use.

Index the characturbtice by 'k', where

$$
k:=0 . .5
$$

Tren

$$
\text { data := READPRN (ascidat) }
$$

Pop $_{1}:=\operatorname{data}_{1,0}$ Population, in thousands

$$
\text { Modules, }:=\operatorname{cell}\left(\frac{P o p_{1}}{10^{3}}\right)
$$

Number of TMC modules

Fusim : $=$ data $_{1.1}$

Number of freeway milles

MinArtig : $=$ data $_{1,2}$ Number of arterial mille

AugArting $_{1}:=\operatorname{data}_{(1,2)} \cdot 5$ Number of (augmented) arterial milloe

Aug := 1 'Aug' lets us conaider the two casean.

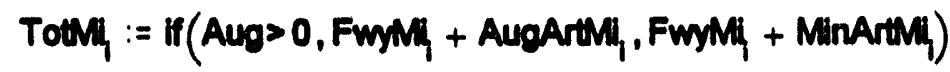

Intreect, $:=I f\left(\right.$ Aug $>0$, data $_{1,3}, 5$, data $\left._{1,3}\right)$

Number of Internections controlled

$$
\text { Geofactor }_{1}:=\text { data }_{1.4}
$$

Factor to adjuat for difiering comatruction coats by climate and-difiering internection denuity

$$
\text { StatusFactor, }_{1}:=\text { data }_{1,3}
$$

Factor to adjust for planning and implemeatation already exinting 
DEFINE UNIT COSTS FOR ARCHITECTURE 3

Costs will be gtven in K (thousandh), M (millions), or B (blilioas)

$$
\text { whore } K=1 \quad M=1000 \cdot K \text { and } B=1000 \cdot M
$$

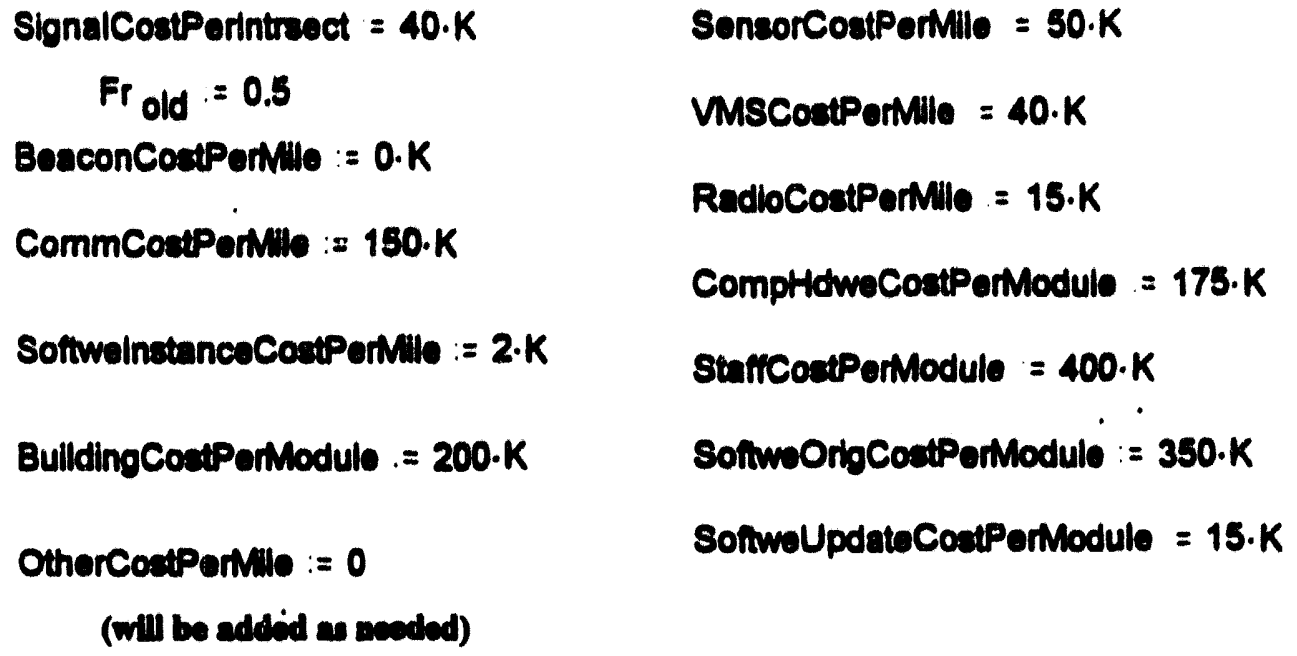

\section{CALCULATE COST RLEMTNTS FOR EACH AREA}

The elements of cooth as Hoted above, can then be calculated for each opectile area.

$$
\begin{aligned}
& \text { ElementCoxt }_{(1,0)}:=0 \quad \text { (will add in as } 10 \% \text { after calenlation of capital cont) }
\end{aligned}
$$

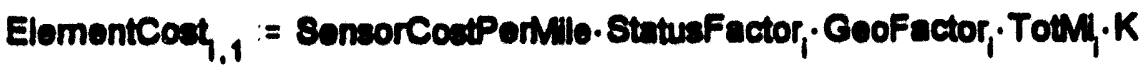

$$
\begin{aligned}
& \text { ElementCost, }:=\text { SinnalCoetPerintreact. }((\overrightarrow{(\text { Intreect, Fr old }+ \text { Fwsily }})) \cdot K \\
& \text { ElementCost, },=\text { VMSContPorMMllo. FuyM, GeoFactor, } \text { StatuaFactor }_{1} \cdot K
\end{aligned}
$$

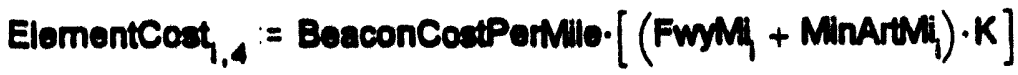

$$
\begin{aligned}
& \text { ElementCost },:=\text { RadioCostPorMilo. FuniM } \cdot K \\
& \text { ElementCost, }:=\text { CommCostPorMilo. Totwi, GeoFactor }, K \\
& \text { ElementCost, }:=\text { CompHdweCostPorModule } \cdot \text { Modules, }, K
\end{aligned}
$$

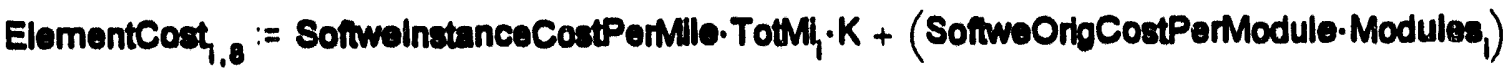

$$
\begin{aligned}
& \text { ElementCost, }:=\text { StaffCostPerModule } \cdot \text { Modules, }, K \\
& \text { ElementCost }, 10_{1}:=\text { Bullding CostPorModule.Modules, } \cdot K \\
& \text { ElementCost }_{(1,11)}:=\text { OtherCostPorMilo.K }
\end{aligned}
$$




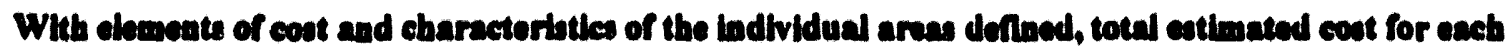
area cea be calculated by summine over the ebments of conth

ImatalledCost $:=\sum_{j}$ ElemontCost $_{1 . j}(j<\theta)$

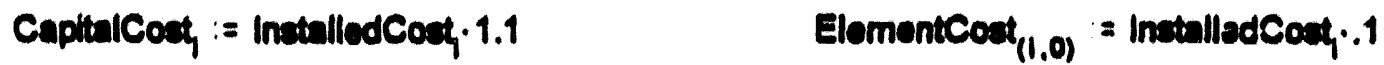

Coaddortas eaglacerteg as $10 \%$ of eapleal cost

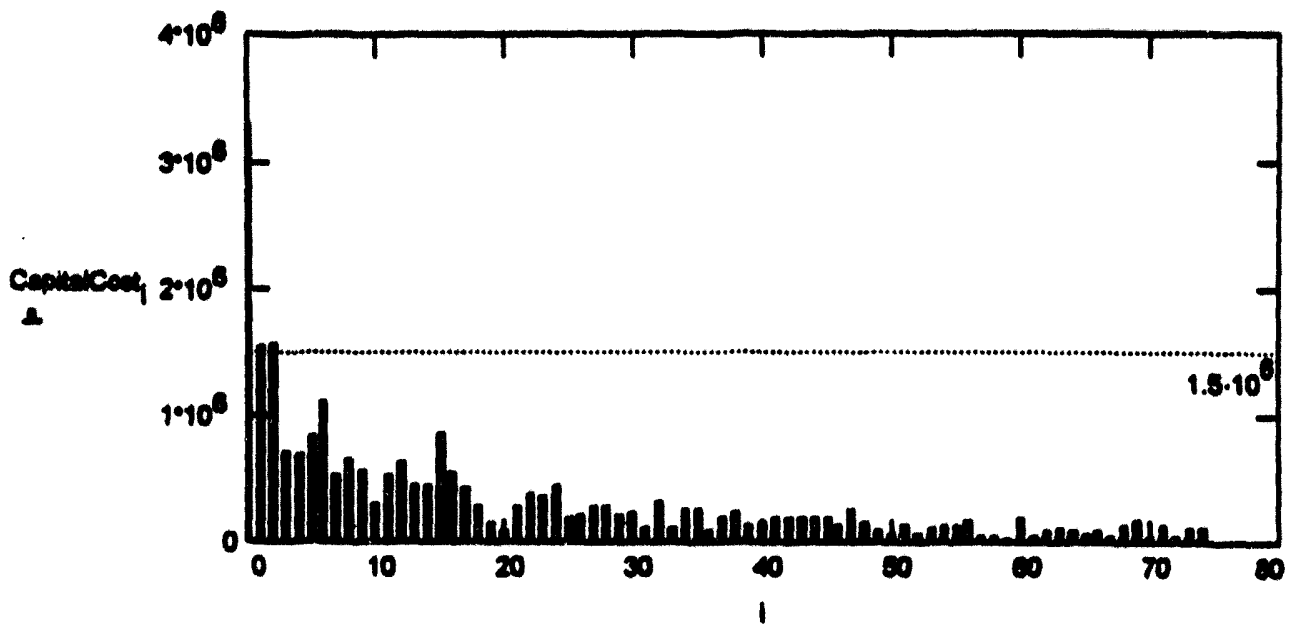

\section{SUM CAPITAL COSTS BY SIER OF ARTAS}

To sum coatu for the largest 9 motropolitan areas, (pop > 4M) we sume from 0 to 8;

$$
\text { LargaAreaCout : }=\sum_{1} \text { CapitalCost } \cdot(1<8)
$$

LargeAreacost $=10.9 \cdot B$

Simillarty,

MediumLargeAreaCoet : $=\sum_{1}$ CapitalCost, $(1>8) \cdot(1<22)$

MediumLargeAreaCost $=5.46 \cdot B$

MediumsmallAreaCost : $=\sum_{1}$ CapitalCoot $\cdot(1>21) \cdot(1<41)$

ModiumSmallAreaCost $=4.32 \cdot B$

SmallAreaCost : $=\sum_{1}$ CapitalCost $\cdot(1>40)$

SmallAreaCost $=3.81 \cdot B$ 
SUM CAPITAL COSTS BY ELEMENT OF COST

ElementCapitalCost $=\sum_{1}$ ElementCoet $_{(1,1)}$

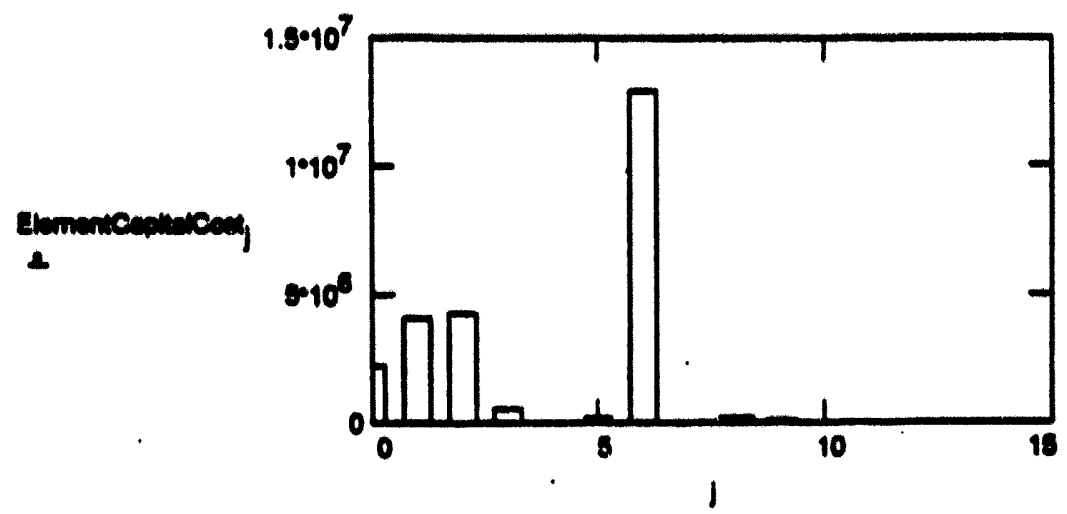

For reforewce, numembor that

clement $0 \mathrm{~b}$ planning and eaghooring cost

cloment 1 is seaver ceeth

cloment 2 ib atganal coeth

clemeat 3 b vartable-measige sign ceoth

clement 4 is besecen cooth

clanent 5 is radto centh

clement 6 b communieation 'eabllng' ceoth

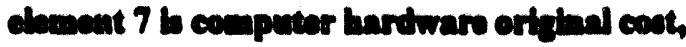

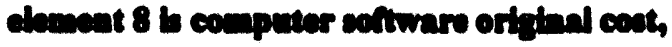

clament 9 is stent coeth

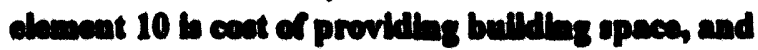

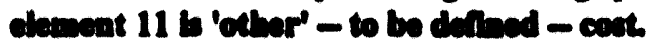

SUM CAPITAL COSTS FOR ALL METROPOLTAN AREAS

To cotimate the grand total capttal cost for all areas, we simply sum the catimated coats for evory area.

$$
\text { GrandTotalCapital := } \sum_{1} \text { Caplalcoat }
$$

With the stated asoumptions, the grand total b

GrandTotalCapital $=24.48 \cdot B$ 


\section{SUM RECURRTG COSTS FOR RDIVDUAL METROPOLTAN ARTAS}

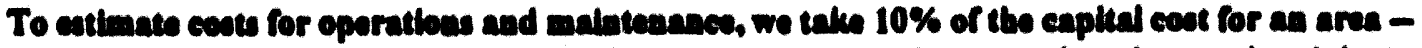

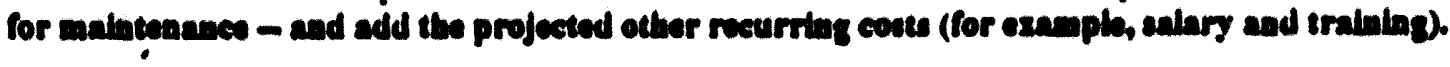

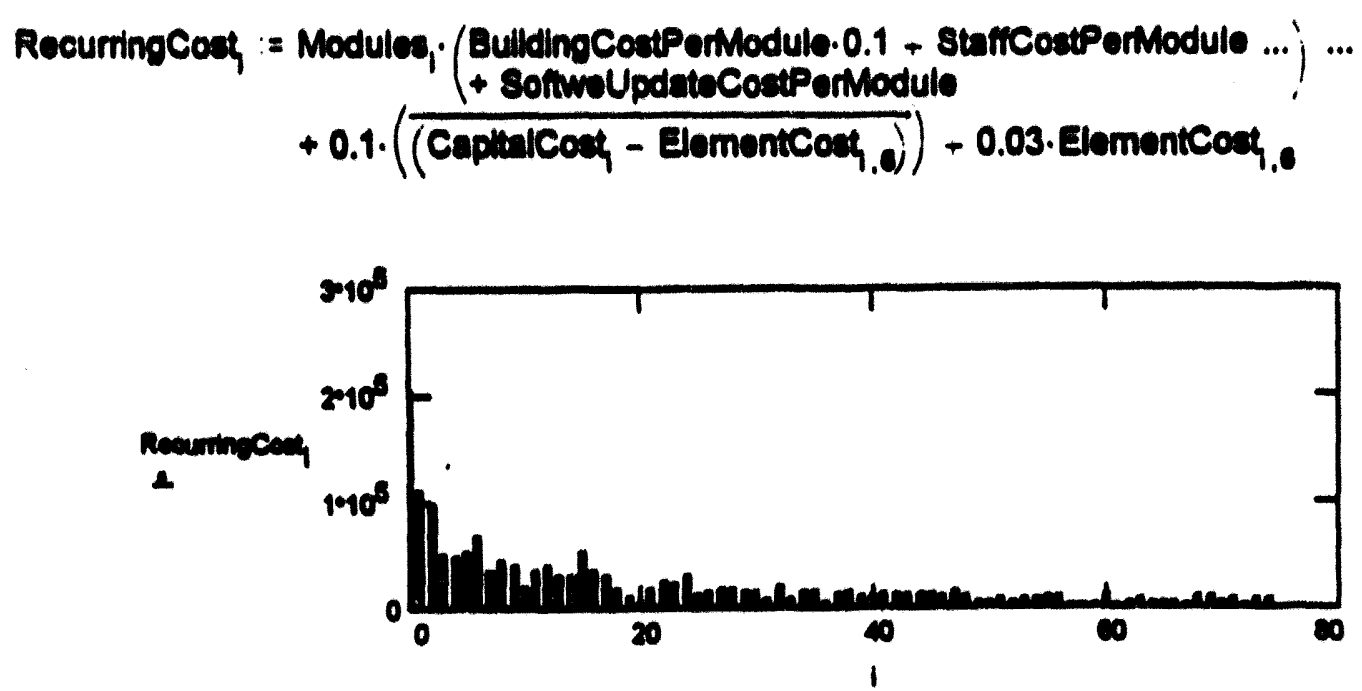

\section{SUM RECURRING COSTS BY SLAE OF ARTA}

$$
\begin{aligned}
& \text { LargaAreaRocurringCoet : }=\sum_{i} \text { RecurringCost. }(i<\theta) \\
& \text { LargeAreaRecuringCost }=710.35 \cdot M \\
& \text { MediumLargaAreaRecurringCost : }=\sum_{1} \text { RecumingCoetp } \cdot(1>8) \cdot(i<22) \\
& \text { ModumLorgoAreaRecurringCoat }=367.75 \cdot M \\
& \text { MediumsmallareaRecuningCost : } \sum_{i} \text { RecuningCost } \cdot(i>21) \cdot(1<41) \\
& \text { MadiumsmallAreaRecumingCost }=289.3 \cdot M \\
& \text { SmallAreaRecurringCost : }=\sum_{1} \text { RecumingCost. }(1>40) \\
& \text { SmallAseaRecurringCost }=258.57 \cdot M \\
& \text { TotalRecumingCont : }=\sum_{1} \text { RecumingCost }
\end{aligned}
$$$$
\text { TotalRiecurringCost }=1.63 \cdot B
$$ 


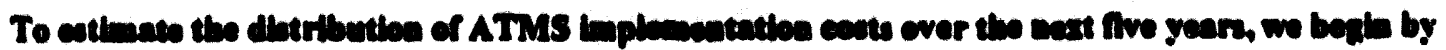

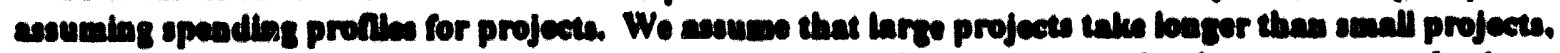

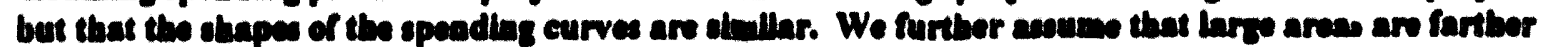

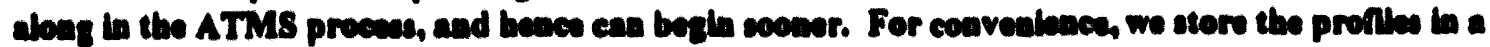

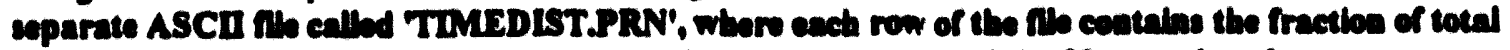

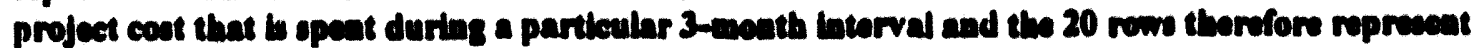

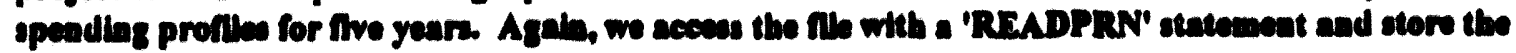
data in a tomporary array.

TimeCoafts : READPRN (timedist pm)

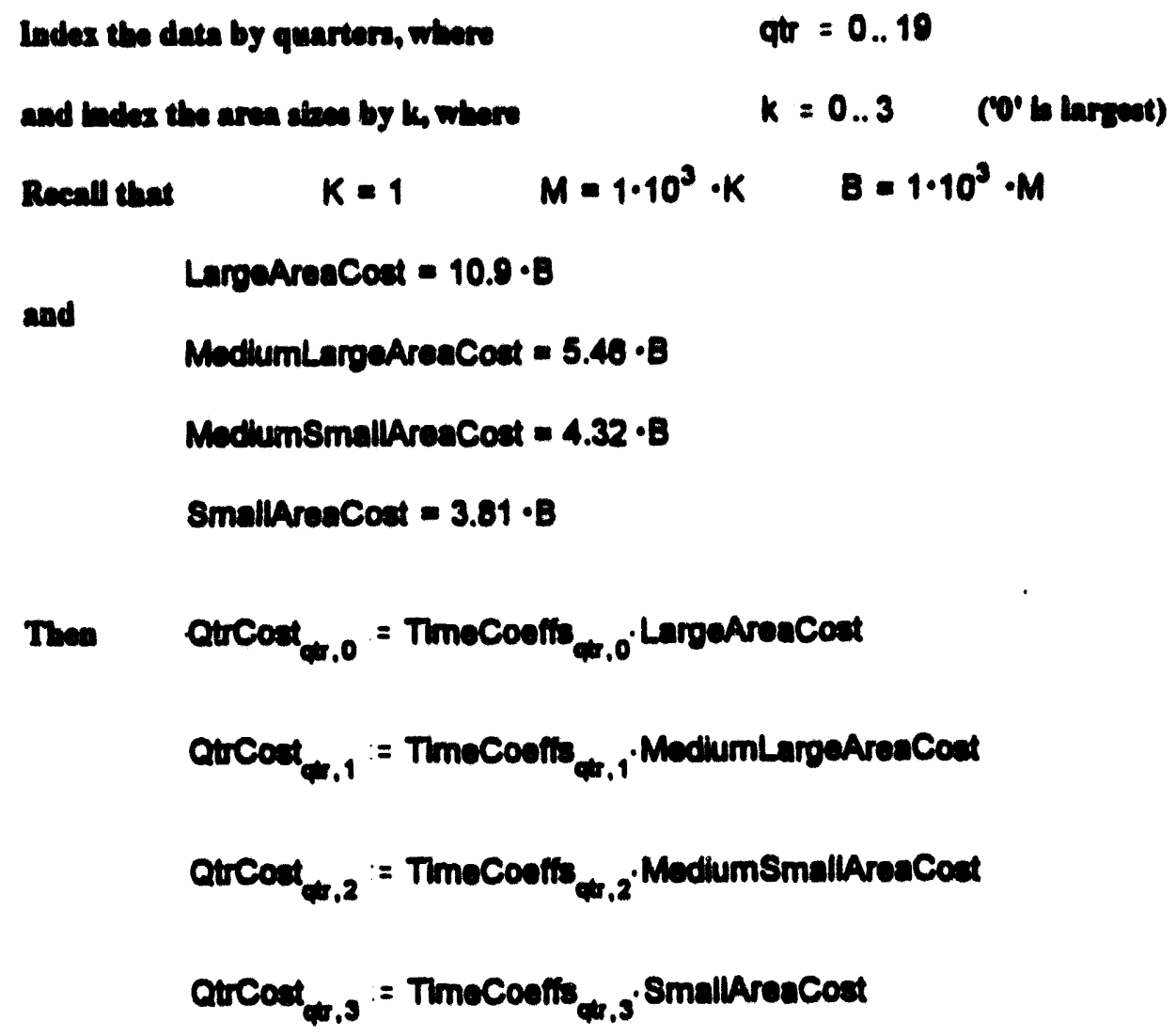

For example,

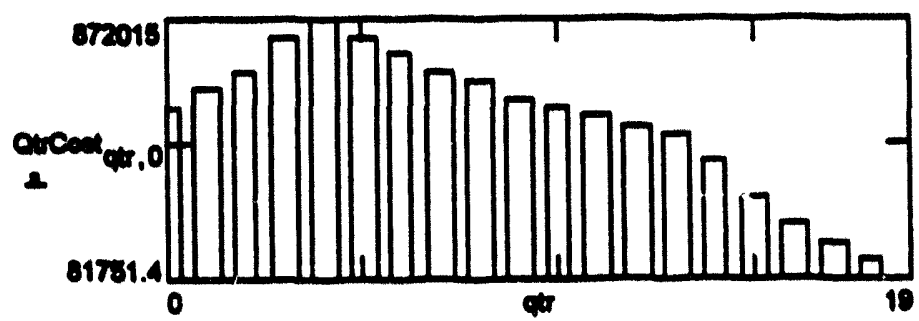

To got total implemeatation cont by year, we sum over the area costs and then sum the quarters in groups of four.

$$
\operatorname{QtrSum}_{\phi}:=\sum_{k} \operatorname{Qutcot}_{\phi, k}
$$


Les yeare be ladesed by $\quad y r=0 . .4$

$$
\begin{aligned}
& \text { Annualcoet }_{0}=\sum_{\text {qtr }} \text { atrsum }_{\phi r} \cdot(\text { qtr< }<\text { ) } \\
& \text { AnnualCost }=2.78 \cdot B \\
& \text { Annuaicoed }_{1}=\sum_{q t} \operatorname{Qtrsum}_{\phi t} \cdot(q t r>3) \cdot(q t r<8)
\end{aligned}
$$

$$
\text { Annualcost }=5.70 \cdot 8
$$

$$
\text { Annualcoet }:=\sum_{\text {qtr }} \operatorname{atrsum}_{\phi} \cdot(\text { qtor } 7) \cdot(\text { qtre } 12) \text {. }
$$

$$
\text { Annualcost }=7.57 \cdot 8
$$

$$
\text { Annualcoot }:=\sum_{q t} \text { Qtrsum }_{\phi i t} \cdot(q t r>11) \cdot(q t<<18)
$$

$$
\text { Annualcost }=5.8 \cdot \mathrm{B}
$$

Annualcoet $_{4}:=\sum_{q t}$ Qtrsum $_{\phi} \cdot(q t r o 15)$

$$
\text { AnnualCost }_{4}=2.53 \cdot B
$$

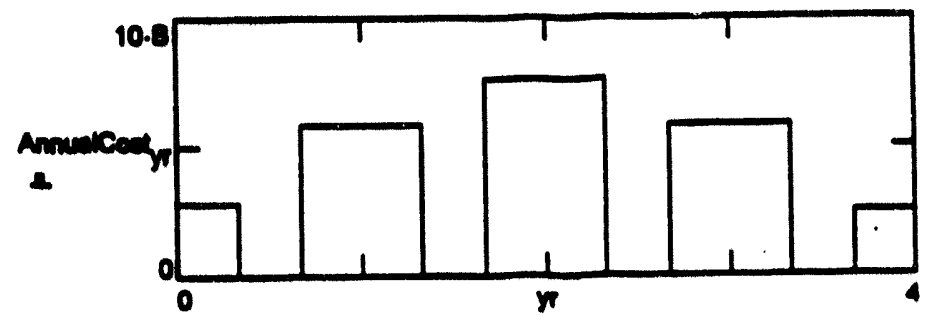

As a chook,

$$
\begin{aligned}
& \text { Sumorcost : }=\sum_{y} \text { AnnualCoet }_{\gamma} \quad \text { SumOrCost }=24.48 \cdot B \\
& \text { GrandTotalCapital }=24.48 \cdot B
\end{aligned}
$$




\section{APPENDEX}

Cost Eotlantes for ATMS Implesentations Computatbal Procedures

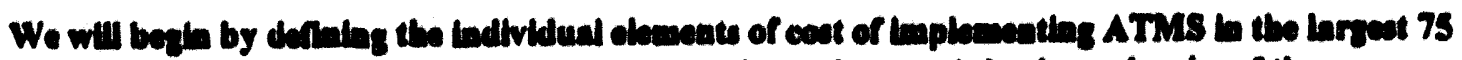

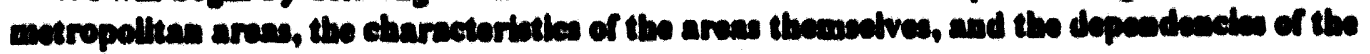

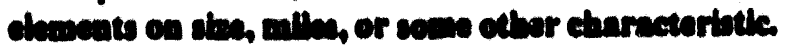

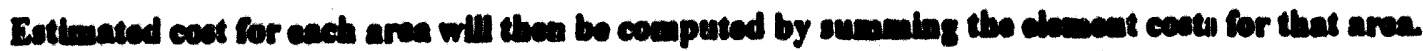

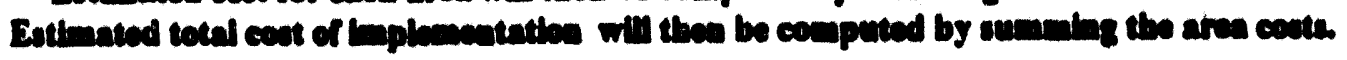

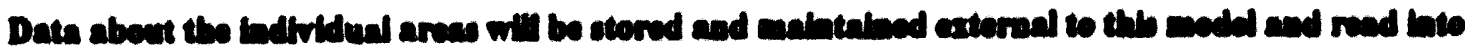

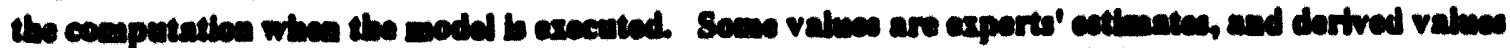

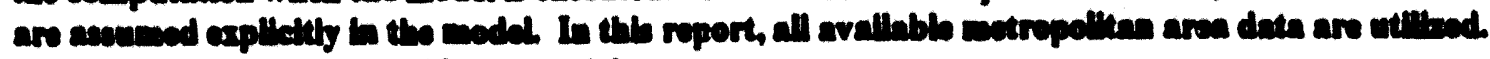
Twe data wit be sterend coteldo the wodal.

\section{MDEX DETIIIONB}

Let the motropellitas aren be ladoed by 'T, whore

$$
\begin{aligned}
& 1:=0.74 \text { and the areas are thoce bleatined in the }
\end{aligned}
$$

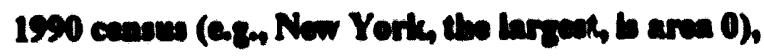

and lat the coet claments by bedered by 'J', where

$$
J:=0 . .11
$$

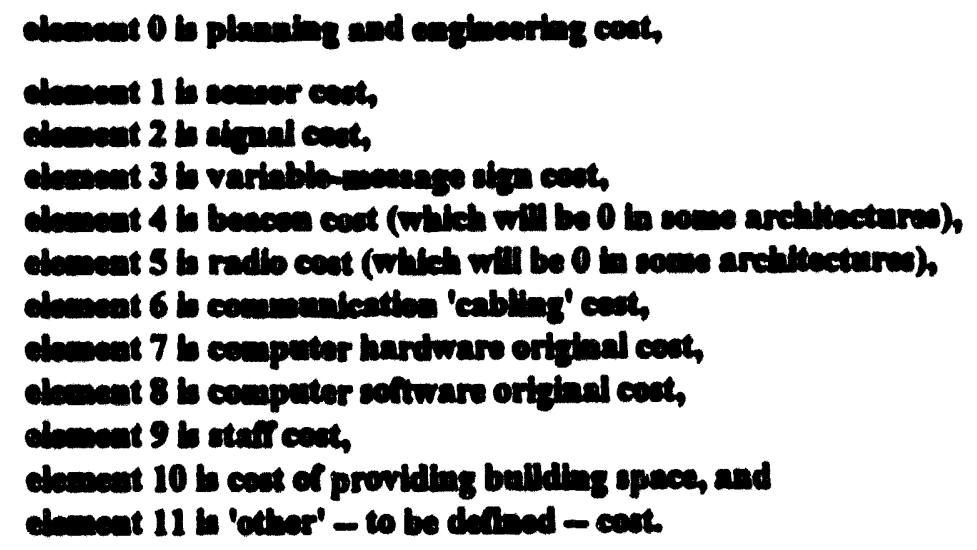

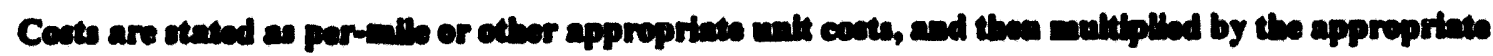
factor to calculate chanont coet for a particular arae. 


\section{READ IN AREA CHARACTERISTIC DATA}

Assume that some of the characteriatics of the metropolitan areas are stored in the rows of an ASCI Nie (named "aceildatprn"), ordered by stze rank, with each row of the fle containing the area's population, number of freeway miles, number of arterial miles, number of signallzed intersections, and adjuatment factors for geographic location and current status of advanced trafilic management planniog or tmplementation. We sceess the Ifle with 'RSAD' statementa. For programming convenience, we'll defiee a data matrits for temporary storage, read into it, and pick out the individual characteristic data for further use.

Index the characteriatica by 'k', where

$$
k:=0 . .5
$$

Then

$$
\text { data := READPRN( asciidat) }
$$

Pop $_{1}:=$ data $_{i, 0}$

$$
\text { Modules }_{i}:=\operatorname{ceil}\left(\frac{P_{0}}{10^{3}}\right)
$$

Fumili $_{i}:=$ data $_{1,1}$

MinArtil : $=$ data $_{1,2}$

Augarting : $=\operatorname{data}_{(1,2)} \cdot 5$

Aug := 0 'Aug' lete us consider the two cacelen.

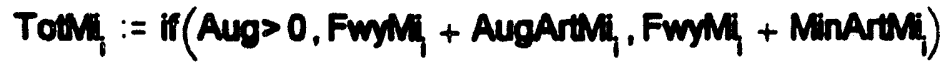

Intreect, : if $\left(\right.$ Aug $>0$, data $_{1,3}, 5$, data $\left._{1,3}\right)$

Number of intersections controlled

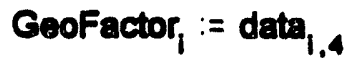

Statusafactor $:=$ data $_{1,5}$
Population, in thousands

Number of TMC modules

Number of freeway milles

Number of arterial miles

Number of (augmented) arterial milles
Factor to adjust for difiering conatrection coets by climate and difiering internoction density

Factor to adjuat for planning and inplementation already exinting 
Costs will be given in $\mathrm{K}$ (thousunda), $\mathbf{M}$ (millions), or B (billions)

$$
\text { where } K=1 \quad M=1000 \cdot K \text { and } B=1000 \cdot M
$$

$$
\begin{aligned}
& \text { SignalCostPerintrsect }=40 \cdot \mathrm{K} \quad \text { SensorCostPerMile }:=50 \cdot \mathrm{K} \\
& \mathrm{Fr}_{\text {old }}=0.5 \\
& \text { BeaconCostPerMile : }=50 \cdot K \\
& \text { CommCostPerMile := } 150 \cdot \mathrm{K} \\
& \text { SoitwelnstanceCostPerMile }:=2 \cdot K \quad \text { StaffCostPerModule }:=400 \cdot K \\
& \text { BuildingCostPerModule }:=200 \cdot K \quad \text { SottweOngCostPerModule }:=350 \cdot K \\
& \text { OtherCostPerNile : }=0 \\
& \text { SoftweUpdateCostPerModule := } 15 \cdot K
\end{aligned}
$$

(will be edded as neoded)

\section{CALCULATE COST ELEMENTS FOR EACH AREA}

The elements of cost, as listed above, can then be exiculated for esch spectile area.

$$
\begin{aligned}
& \text { ElementCost }_{(1,0)}:=0 \quad \text { (will add in as } 10 \% \text { after calculation of capital cont) }
\end{aligned}
$$

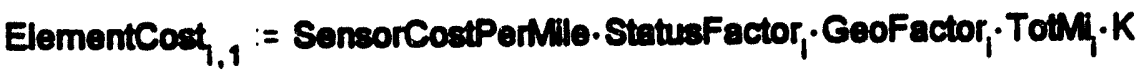

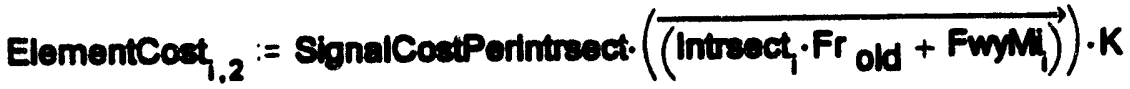

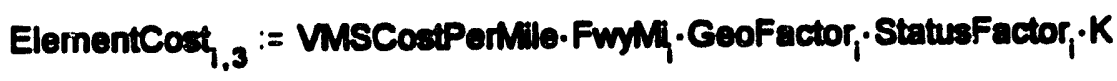

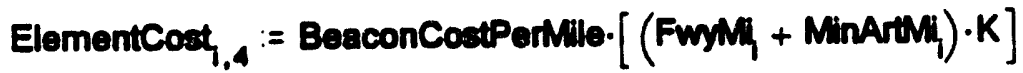

$$
\begin{aligned}
& \text { ElementCost },:=\text { RadioCostPerivilo. FwyMly.K } \\
& \text { ElementCost } 1,=\text { CommCostPerMile.TotMi, GeoFactor } \cdot K \\
& \text { ElementCost }\}_{.7}:=\text { CompHdweCostPerModule.Modules, } K \\
& \text { ElementCost }, \text { := SoftwelnstanceCostPerMile } \cdot \text { TotMi } \cdot K+(\text { SoftweOrigCostPerModule } \cdot \text { Modules, }) \\
& \text { ElementCost }, 0:=\text { StaffCostPerModulo-Modules, } K \\
& \text { ElementCost }, 10:=\text { BulldingCostPerModule } \cdot \text { Modules, } \mathrm{K} \\
& \text { ElementCost }_{(1,11)}:=\text { OtherCostPerMile.K }
\end{aligned}
$$


With elements of coat and characteriatica of the individual areas defined, total eatimated cont for each area can be calculated by summing over the elements of cost.

$$
\text { InstalledCost }:=\sum_{j} \text { ElementCost }, j(j<\theta)
$$

CapitalCost $:=$ InstalledCost, $1.1 \quad$ ElementCost $(1,0):=$ InstalledCost, $\cdot 1$

\section{Conoldering engincering as $10 \%$ of} capital cont

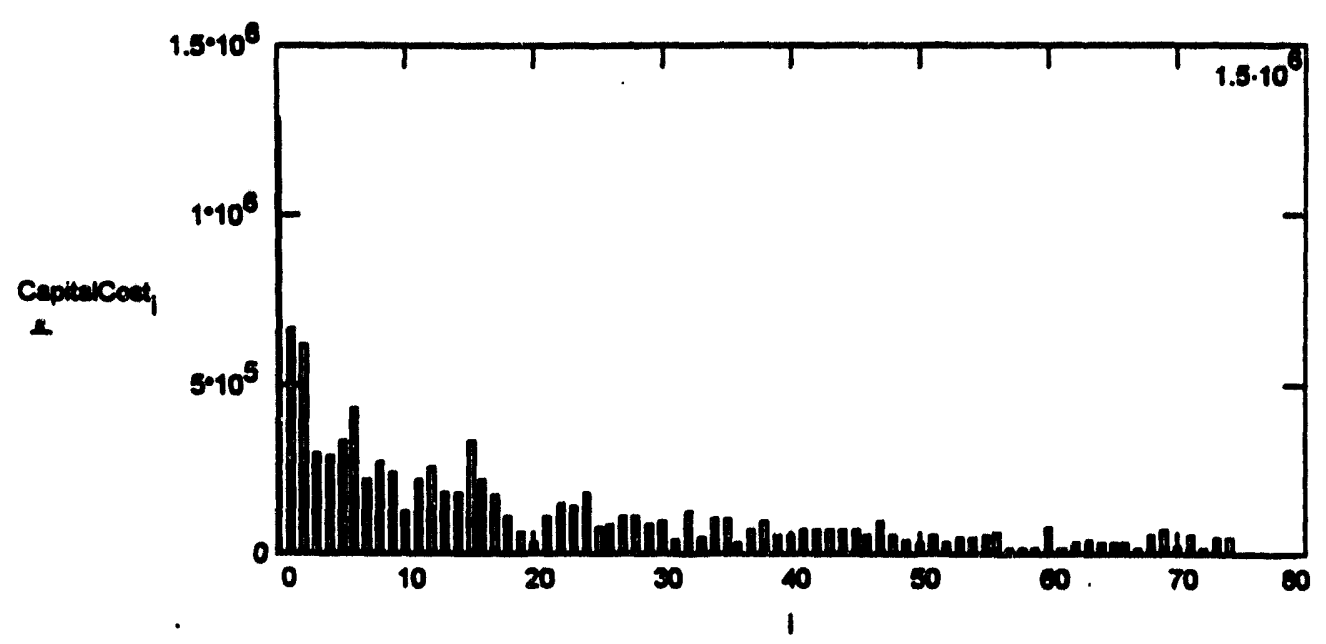

\section{SUM CAPITAL COSTS BY SIZT: OF AREAS}

To sum costs for the larzeat 9 metropolitan areas, (pop > 4M) we sum from 0 to 8;

$$
\text { LargeAreaCost : }=\sum_{i} \text { CapitalCost } \cdot(i<9)
$$

$$
\text { LargeAreaCost }=4.41 \cdot B
$$

Similarty,

$$
\begin{aligned}
& \text { MediumLargeAreaCost }:=\sum_{i} \text { CapitalCost } \cdot(i>8) \cdot(i<22) \\
& \text { MediumLargeAreaCost }=2.25 \cdot B \\
& \text { MediumSmallAreaCost }:=\sum_{i} \text { CapitalCost } \cdot(i>21) \cdot(i<41) \\
& \text { SmallAreaCost }:=\sum_{i} \text { CapitalCost } \cdot(i>40) \\
& \text { SediumSmallAreaCost }=1.76 \cdot B
\end{aligned}
$$


ElementCapitalCost $_{\}}=\sum_{i}$ ElementCost $_{(1,1)}$

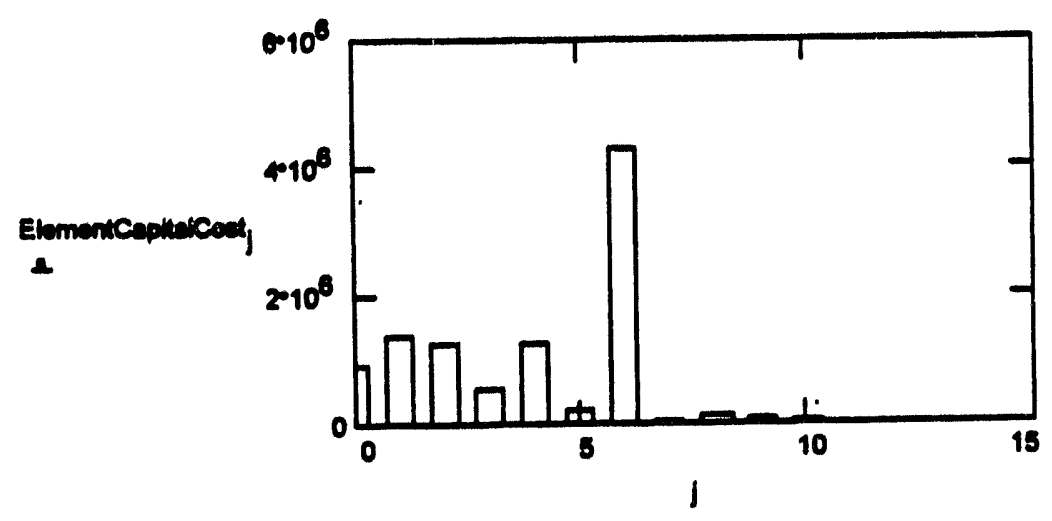

For reference, remember that

element 0 is planning and engineertag cost

element 1 is eensor costh

element 2 is signal coot,

element 3 b variablo-meanage sign cont,

element 4 is beacen cont,

element 5 is radlo coeth,

element 6 is communication 'cabling' cost,

element 7 is computer hardware original cost.

dement 8 b computor soltware original cooth,

element $9 \mathrm{~b}$ staft cont,

clement $10 \mathrm{~b}$ cost of providing bullding spacen and

element 11 ib 'other' - to be defled - cost.

\section{SUM CAPITAL COSTS FOR ALL METROPOLTAN AREAS}

To estimate the grand total capital cont for all areas, we simply sum the eatimated costs for every area.

$$
\text { GrandTotalCapital : }=\sum_{i} \text { CapitalCost }
$$

With the stated assumptions, the grand total is

$$
\text { GrandTotalCapital }=9.97 \cdot B
$$


To estimate costs for operations and maintenance, we take $10 \%$ of the capital cost for an area for maintenance - and add the projected other recurring costs (for example, salary and training).

RecurringCost $:=$ Modules $;\left(\begin{array}{l}\text { BuildingCostPerModule.0.1 + StaffCostPerModule ... } \\ + \text { SoftweUpdateCostPerModule }\end{array}\right)$... $+0.1 \cdot(\overrightarrow{(\text { CapitalCost }- \text { ElementCost } 1.6}))+0.03 \cdot$ ElementCost $_{1.6}$

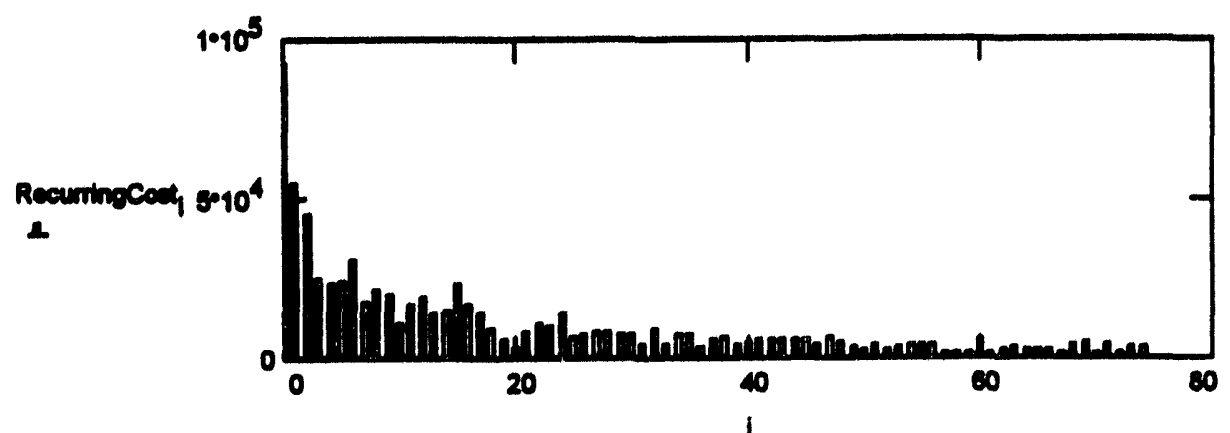

\section{SUM RECURRING COSTS BY SIZE OF AREA}

LargeAreaRecurringCost : $=\sum_{i}$ RecurringCost, $(i<\theta)$

LargeAreaRecurringCost $=335.43 \cdot M$

MediumLargeAreaRecurringCost := $\sum_{1}$ RecurringCost. $(i>8) \cdot(i<22)$

MediumLargeAreaRecurringCost $=176.77 \cdot M$

MediumSmallAreaRecurringCost := $\sum_{i}$ RecurringCost $\cdot(i>21) \cdot(i<41)$

ModiumSmallAreaRecurringCost $=138.69 \cdot M$

SmallAreaRecurringCost : $=\sum_{i}$ RecurringCost. $(i>40)$

SmallAreaRecurringCost $=125.42 \cdot M$

TotalRecurringCost : $=\sum_{i}$ RecurringCost

TotalRecurringCost $=0.78 \cdot B$

Arch 4 Min Network 
To extimate the distribution of ATMS implementation costs over the next five yearr, we begin by assuming speading proftie for projects. We assume that large projects take longer than small projects, but that the shapes of the spending curves are similar. We further assume that large areas are farther along in the ATMS process, and hence can begin sooner. For convenience, we store the proflibes in a separate ASCD Ite callod TIMEDDIST.PRN', where each row of the file contains the fraction of total project cost that is apent during a particular 3-month interval and the 20 rows therefore represent speading profties for five years. Agaln, we access the file with a 'READPRN' statement and atore the data in a temporary array.

$$
\text { TimeCoeffs : = READPRN (timodist pm) }
$$

$$
\begin{aligned}
& \text { Index the data by quarters, where } \\
& q \text { qr }=0 . .19 \\
& \text { and inder the area stees by } k \text {, where } \\
& k:=0 . .3 \quad\left({ }^{\prime} \text { is largest }\right) \\
& \text { Recall that } \quad K=1 \quad M=1 \cdot 10^{3} \cdot K \quad B=1 \cdot 10^{3} \cdot M
\end{aligned}
$$

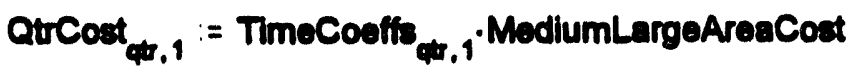

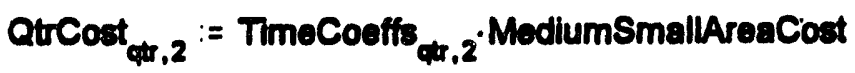

$$
\begin{aligned}
& \text { QtrCost }_{\phi t, 3}:=\text { TimeCoeffs } \text { q }, 3_{3} \cdot \text { SmallAreaCost }
\end{aligned}
$$

For exumples,

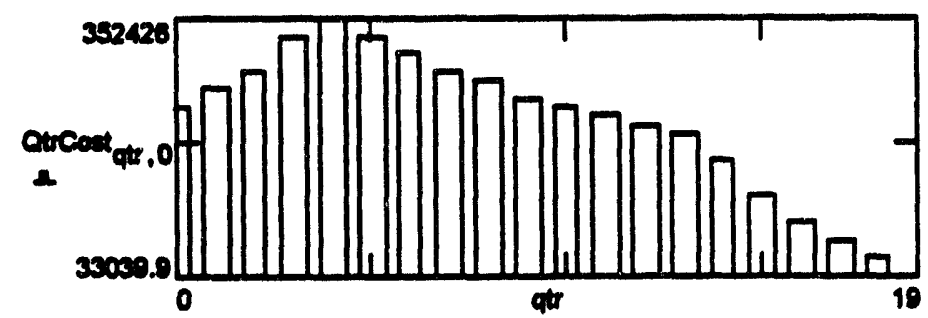

To get total implementation cost by year, we sum over the area costs and then sum the quarters in groups of four.

$$
\text { QtrSum }_{\phi r}:=\sum_{k} \text { QtrCost }_{\phi, k}
$$




$$
\text { AnnualCost }_{0}=\sum_{\text {qtr }} \text { Qtrsum }_{\text {qut }} \cdot(\text { qtr< }<4)
$$

$$
\text { AnnualCost } 0=1.12 \cdot B
$$

AnnualCost $_{1}:=\sum_{\text {qtr }}$ QtrSum $_{\text {qu }} \cdot($ qtro 3$) \cdot($ qtr $<8)$

AnnualCost, $=2.36 \cdot B$

$$
\text { AnnualCost }:=\sum_{q t r} \text { QtrSum }_{\text {qur }} \cdot(\text { qtr> } 7) \cdot(\text { qtr< } 12)
$$

AnnualCost $2=3.09 \cdot B$

$$
\text { AnnualCost }_{3}:=\sum_{q t r} \text { QtrSum }_{q t r} \cdot(\text { qtr> 11) } \cdot(\text { qtr< } 16)
$$

AnnualCost $_{3}=2.37 \cdot B$

$$
\text { AnnualCost }_{4}:=\sum_{q t r} \text { QtrSum }_{\text {qt }} \cdot(q t r>15)
$$

$$
\text { AnnualCost }_{4}=1.03 \cdot B
$$

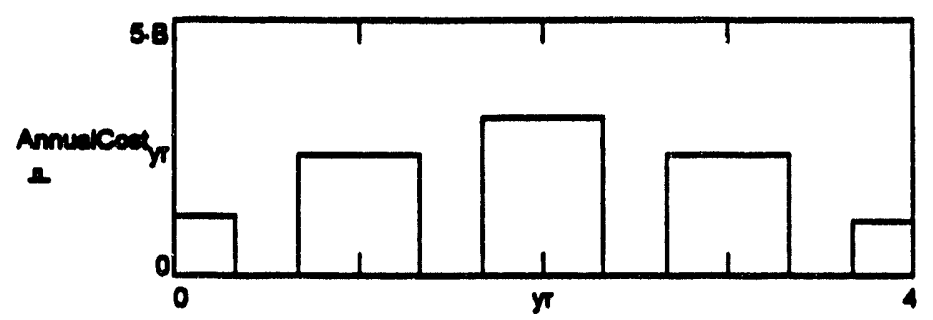

As a check,

$$
\begin{aligned}
& \text { SumorCost : }=\sum_{y r} \text { AnnualCost }_{y r} \quad \text { SumOfCost }=9.97 \cdot B \\
& \text { GrandTotalCapital }=9.97 \cdot B
\end{aligned}
$$


We will begin by dentaing the individual elemeats of cost of Implementing ATMS in the largeat 75 metropolitan areas, the characteriticas of the areas themeelves, and the dependencies of the elemeats on abe, milles, or some other characteriatic.

Estimated cost for each ares will then be computed by summing the element costs for that area. Estimated total cost of lmplomentation will then be computed by summing the area costs.

Date about the ledividual areas will be stored and malntalned external to thls model and read into the computation when the modal b exceuted. Some values are experts' eatimates, and derfved values are asaumed explicitly in the model. In this report, all avallable motropolltas area data are utilleod. The data will be etorod outelde the model.

\section{INDEX DEFINITONS}

Lot the metropolitan areas be indered by 'I', where

$$
\begin{aligned}
& 1:=0.74 \text { and the areas are those identified in the } \\
& 1990 \text { ceasus (as.o. New York, the largeat, w area 0), }
\end{aligned}
$$

and let the coat elements by ladered by 'J', where

$$
j:=0 . .11
$$

element 0 is planning and engineoring cost,

eloment 1 be censer costh

element 2 is slomal conth

element 3 b variablo-measage algen costh

element 4 b beecon coit (which will be 0 in come archittecturea),

element 5 bi radio cont (which will be 0 the come architectures),

element 6 b communication 'cabling' cost,

element 7 is computer hardware orifinal cosh

element 8 is computer eoftrare original cost,

element 9 b stafi costh

element 10 b coat of providing bulldtag apace, and

element 11 is 'other' - to be defined - coot.

Costs are atated as per-mile or other appropriate unit couts, and then multiplied by the appropriate factor to calculate element cost for a particular area. 


\section{READ IN AREA CHARACTERISTIC DATA}

Assume that some of the characteriatics of the motropolltan areas are stored in the rows of an ASCU The (asamed "asetldat.prn"), ordered by stze raak, with each row of the flle coutaining the area's population, number of frewway miles, number of arterial milba, number of algnallead internections, and adjustment factor for geograptic location and current status of advanced trafile management planning or implementation. We aceses the flle with 'READ' statemeats. For programming convenience, we'll define a data matris for temporary storage, read into $\mathrm{it}$, and plek out the individual characteriatle data for further use.

Index the charactartatiea by 'k', whore

$$
k:=0 . .5
$$

$$
\begin{aligned}
& \text { Then data := READPRN( asciidat) } \\
& \text { Pop }:=\text { data }_{1,0} \\
& \text { Modules, }=\operatorname{cell}\left(\frac{P_{0 p_{1}}}{10^{3}}\right) \\
& \text { Population, in thousands } \\
& \text { Frymili }_{1}:=\text { data }_{1,1} \\
& \text { Number of freeway milles } \\
& \text { MinAruM : }=\text { data }_{1,2} \\
& \text { Number of arterial his is } \\
& \text { AugArtili, : }=\operatorname{data}_{(1,2)} \cdot 5 \\
& \text { Number of (augmented) suterial milles } \\
& \text { Aug : = } 1 \text { 'Aug' lets us consider the two caseam } \\
& \text { TotMi }:=I r\left(\text { Aug }>0, F w M M_{1}+\text { AugArtM }, \text { FwyMl }+ \text { MinArtMi }\right) \\
& \text { Intreect } 1:=\text { If }\left(\text { Aug }>0, \text { data }_{1,3}, 5, \text { data }_{1,3}\right)
\end{aligned}
$$

Number of latersections controlled

$$
\text { Geofactor, }_{1}=\text { data }_{1,4}
$$

Factor to adjuat for difiering construction conts by climate and difiering internection density

StatusFactor,$:=$ data $_{1,5}$

Factor to adjust for planning and implementation already exiating 


\section{DEFUNE UNTT COSTS FOR ARCHITECTURE $\downarrow$.}

Costs will be given in $\mathbf{K}$ (thousands), $\mathbf{M}$ (millioas), or $\mathbf{B}$ (blltioas)

$$
\text { where } K=1 \quad M=1000 \cdot K \text { and } B=1000 \cdot M
$$

$$
\begin{aligned}
& \text { SignalCostPorintreact }=40 \cdot \mathrm{K} \\
& \text { SensorCostPerMile }=50 \cdot K \\
& F_{\text {old }}=0.5 \\
& \text { BeaconCostPermillo : }=50 \cdot \mathrm{K} \\
& \text { CommCostPerMille }:=150 \cdot \mathrm{K} \\
& \text { SottwelnatancocostPorMile : }=2 \cdot K \quad \text { StaffCostPorModule }=400 \cdot K \\
& \text { BulldingCostPerModule }:=200 \cdot \mathrm{K} \quad \text { SotwwoOngCostPerModule }=350 \cdot \mathrm{K} \\
& \text { OthercostPertivile: }=0 \\
& \text { SoftwolupdateCostPerModule }:=15 \cdot \mathrm{K}
\end{aligned}
$$

\section{CALCULATE COST ELEMENTS FOR EACH AREA}

The elements of coot, as llted above, can then be calculoted for each spectile area.

$$
\begin{aligned}
& \text { ElementCost }_{(1,0)}:=0 \quad \text { (will add in as } 10 \% \text { after calculation of capital cost) }
\end{aligned}
$$

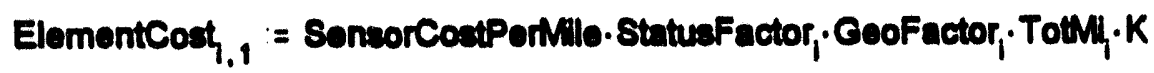

$$
\begin{aligned}
& \text { ElementCost, }:=\text { SignalCoetPerintreect }_{1,2}\left(\overrightarrow{\left(\text { Intreect, } F r_{\text {old }}+\text { FwMMil }\right)}\right) \cdot K
\end{aligned}
$$

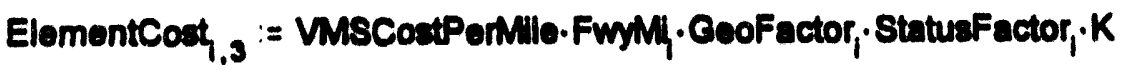

$$
\begin{aligned}
& \text { ElementCost }, 4:=\text { BeaconCostPerMile } \cdot[(\text { FwsMil }+ \text { MinArtMi }) \cdot K] \\
& \text { ElementCost, }:=\text { RadioCostPerMillo. FwMM. } K \\
& \text { ElementCost, }=\text { CommCostPerMilo. TotM } \cdot \text { GeoFactor }, K
\end{aligned}
$$

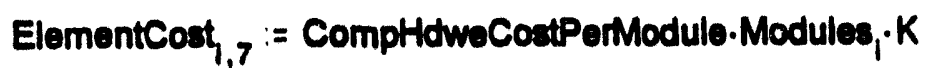

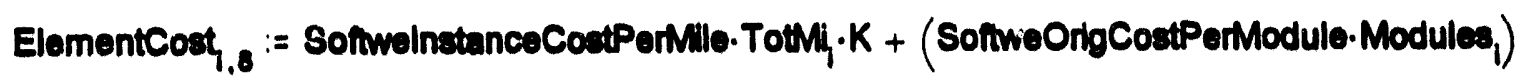

$$
\begin{aligned}
& \text { ElementCost }, 0=\text { StafrCostPorModule } \cdot \text { Modules, } \cdot K \\
& \text { ElementCost }, 10:=\text { BuildingCostPerModule } \cdot \text { Modules }_{1} \cdot K \\
& \text { ElementCost }_{(1,11)}:=\text { OtherCostPerMillo.K }
\end{aligned}
$$




\section{SUM CAPITAL COSTS FOR THE INDIVDUAL METROPOLTTAN AREAS}

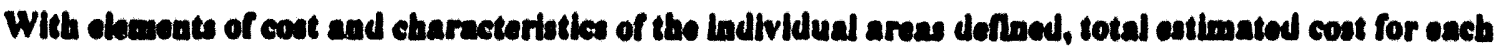
area can be calculated by summing over the eloments of cost.

$$
\begin{aligned}
& \text { InstalledCost }:=\sum_{j} \text { ElomentCost }_{1, j}(j<\theta) \\
& \text { CapitalCost }:=\text { InstalledCost } \cdot 1.1 \quad \text { ElomentCost }(1,0)=\text { InstalledCost, } \cdot 1
\end{aligned}
$$

\section{Comoldortag englecortang as $10 \%$ of} capltal cont

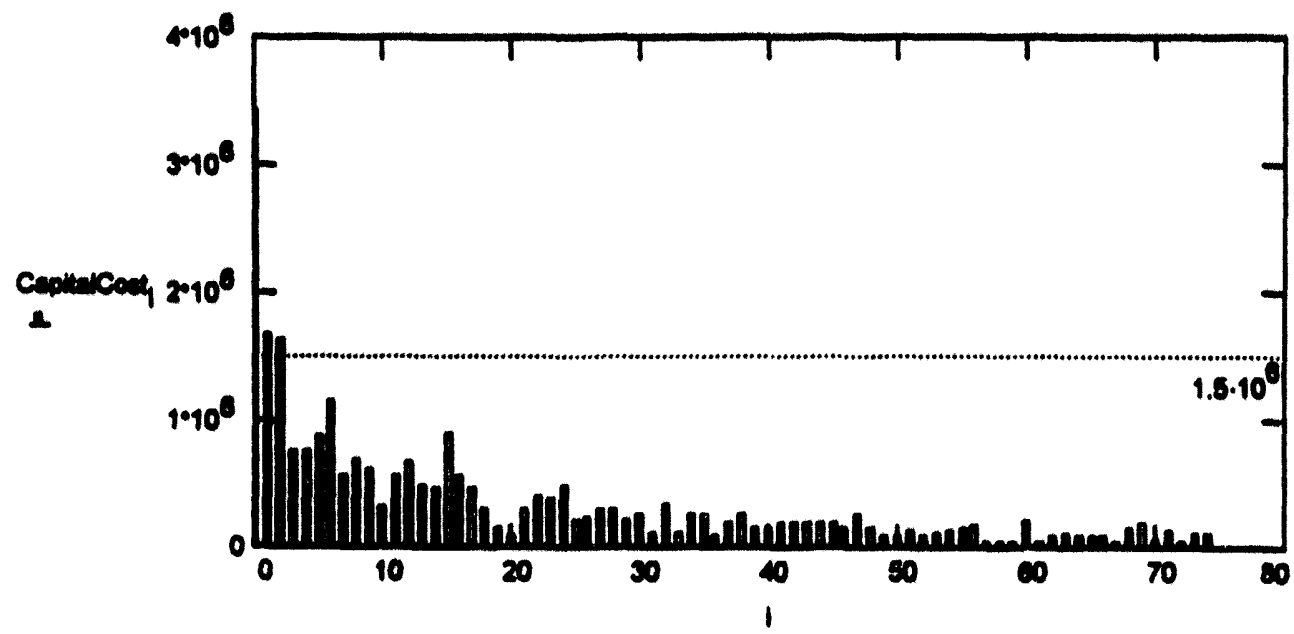

\section{SUM CAPITAL COSTS BX SIET OF AREAS}

To sum coats for the largent 9 metropolltan areas, (pop > 4M) we sum from 0 to 8 ;

$$
\text { LargeAreaCoot : }=\sum_{1} \text { CapltalCost. }(1<\theta)
$$

$$
\text { LargeAreaCost }=11.49 \cdot B
$$

Similarty,

$$
\begin{aligned}
& \text { MediumLargeAreaCost }:=\sum_{i} \text { CapitalCost, }(1>8) \cdot(1<22) \\
& \text { MediumLargeAreaCost }=5.79 \cdot B \\
& \text { MediumSmallAreaCost }:=\sum_{i} \text { CapitalCost } \cdot(1>21) \cdot(1<41) \\
& \text { SmallAreaCost }:=\sum_{1} \text { CapitalCost, } \cdot(1>40) \\
& \text { SmallAreaCost }=4.03 \cdot B
\end{aligned}
$$




$$
\text { ElomontCapitalCost }_{1}=\sum_{1} \text { ElementCost }_{(1,1)}
$$

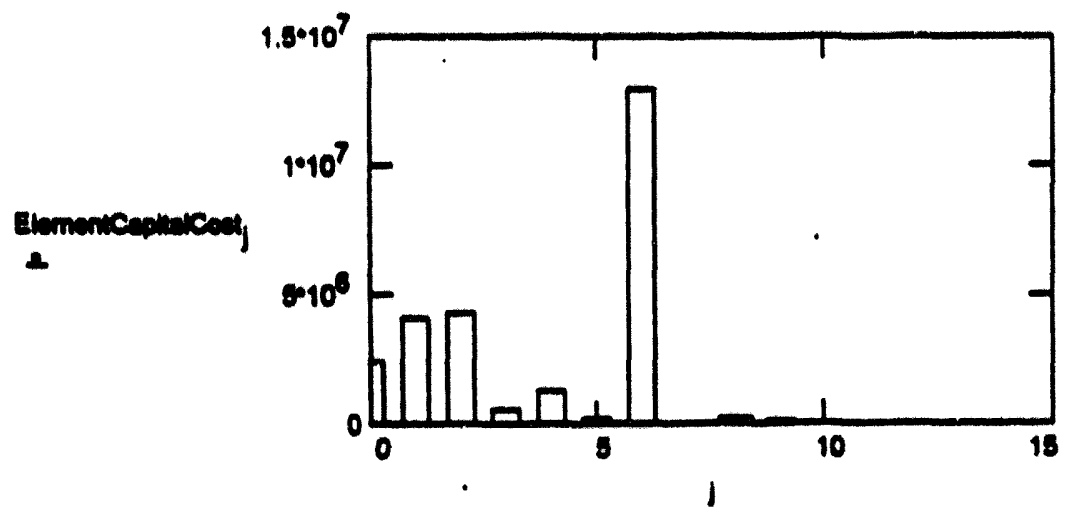

For reforence, remenbor that

element 0 bs planalug and engincortag cost

element 1 b eaver costh

cloment 2 b algeal coot.

cloment 3 b variable-weacage slege costh

eloment $4 \mathrm{~b}$ bescose ceeth.

dement 5 b radto cooth

element 6 b communication 'cabllag' cost,

element 7 b computer hardware ortiginal cost,

eleweent 8 b computar soltware ortighel conth

clement 9 b otafr coeth

elemeat $10 \mathrm{~b}$ cost of providiag bullding apaces, and

elomeat 11 b 'othor' - to be drifined - coeth

\section{SUM CAPITAL COSTS FOR ALL METROPOLTTAN AREAS}

To estimate the grand total capital cost for all areas, we simply sum the eatimated costs for every area.

$$
\text { GrandTotalCapital : }=\sum_{1} \text { CepitalCost }
$$

With the stated asumptions, the grand total b

GrandTotalCapital $=25.88 \cdot B$

Arch 4 Augmented Network 
To entimate costu for oporations and maintenance, wo take $10 \%$ of the capital cont for an area for mainteasece - and add the projected ocher recurring cents (for examplo, sulary and tralabog).

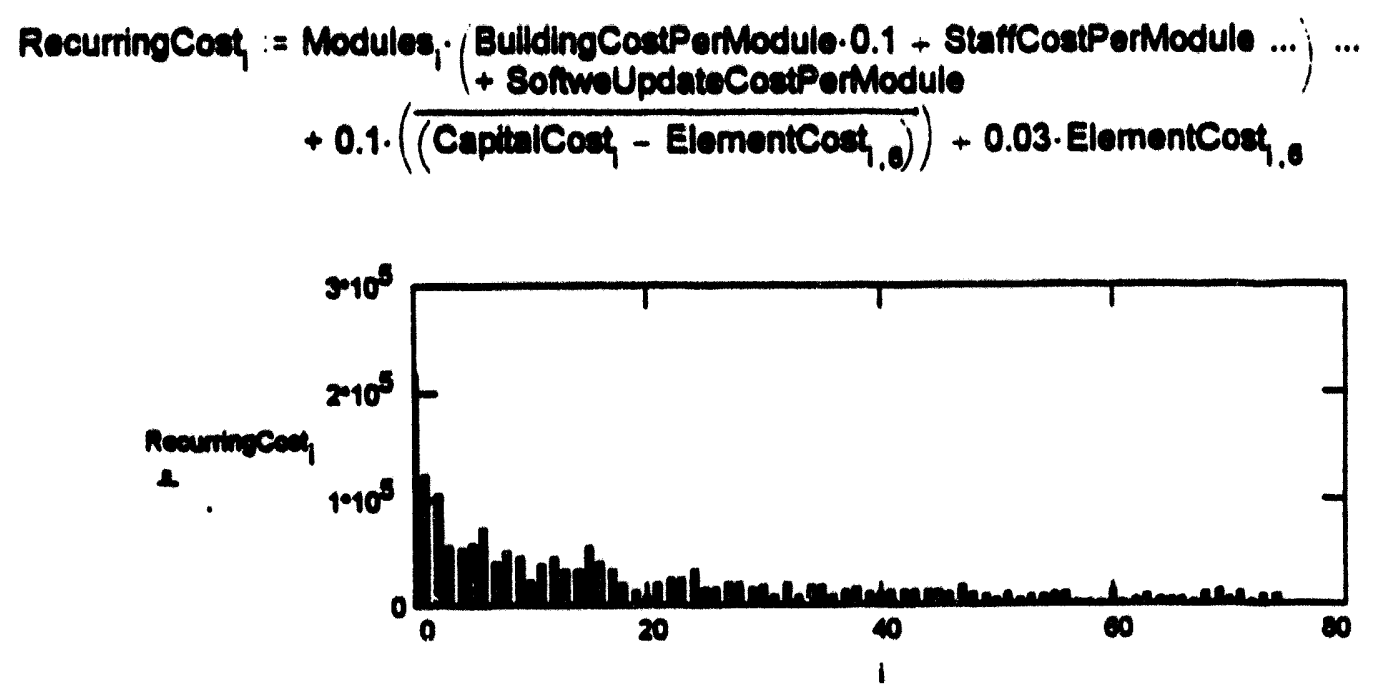

SUM RECURRING COSTS BY SIZE OF AREA

$$
\begin{aligned}
& \text { LargeAreaRecurringCost : } \sum_{i} \text { RecurringCost. }(1<\theta) \\
& \text { LargeAreaRecuringCoat }=760.2 \cdot M \\
& \text { ModlumLargeAreaRecurringCoat : }=\sum_{1} \text { RecuningCost, }(1>8) \cdot(1<22) \\
& \text { ModiumLargeAreaRecurringCost }=400.91 \cdot M \\
& \text { MediumsmallareaRecurringCost := } \sum_{1} \text { RecumingCost. }(1>21) \cdot(1<41) \\
& \text { MedlumsmallAreaRecurringCost }=313.17 \cdot \mathrm{M} \\
& \text { SmallAreaRecurringCost : }=\sum_{1} \text { RecurringCost } \cdot(i>40) \\
& \text { SmallAreaRecurringCost }=281.01 \cdot M \\
& \text { TotalRecurringCost : }=\sum_{i} \text { RecurringCost } \\
& \text { TotalRecurringCost }=1.76 \cdot B
\end{aligned}
$$




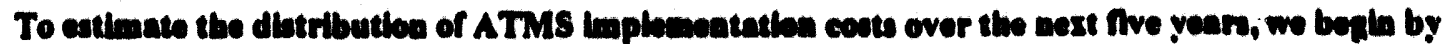

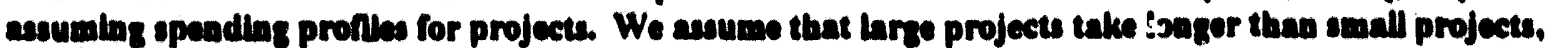
but that the shapen of the speadiag curves are simillar. We further sasume that large areas are farthor

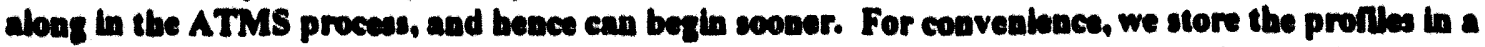
separate ASCII the callod TIMEDIST.PRN', where each row of the file contalas the fraction of total project cost that is speat durtag a particular 3-month interval and the 20 rows therefore represent spendling profles for five yearn. Agatn, we sceses the fllo with a 'READPRN' statemeat and store the data in a temporary array.

$$
\text { TimoCooffe }=\text { READPRN }(\text { timodiat pm })
$$

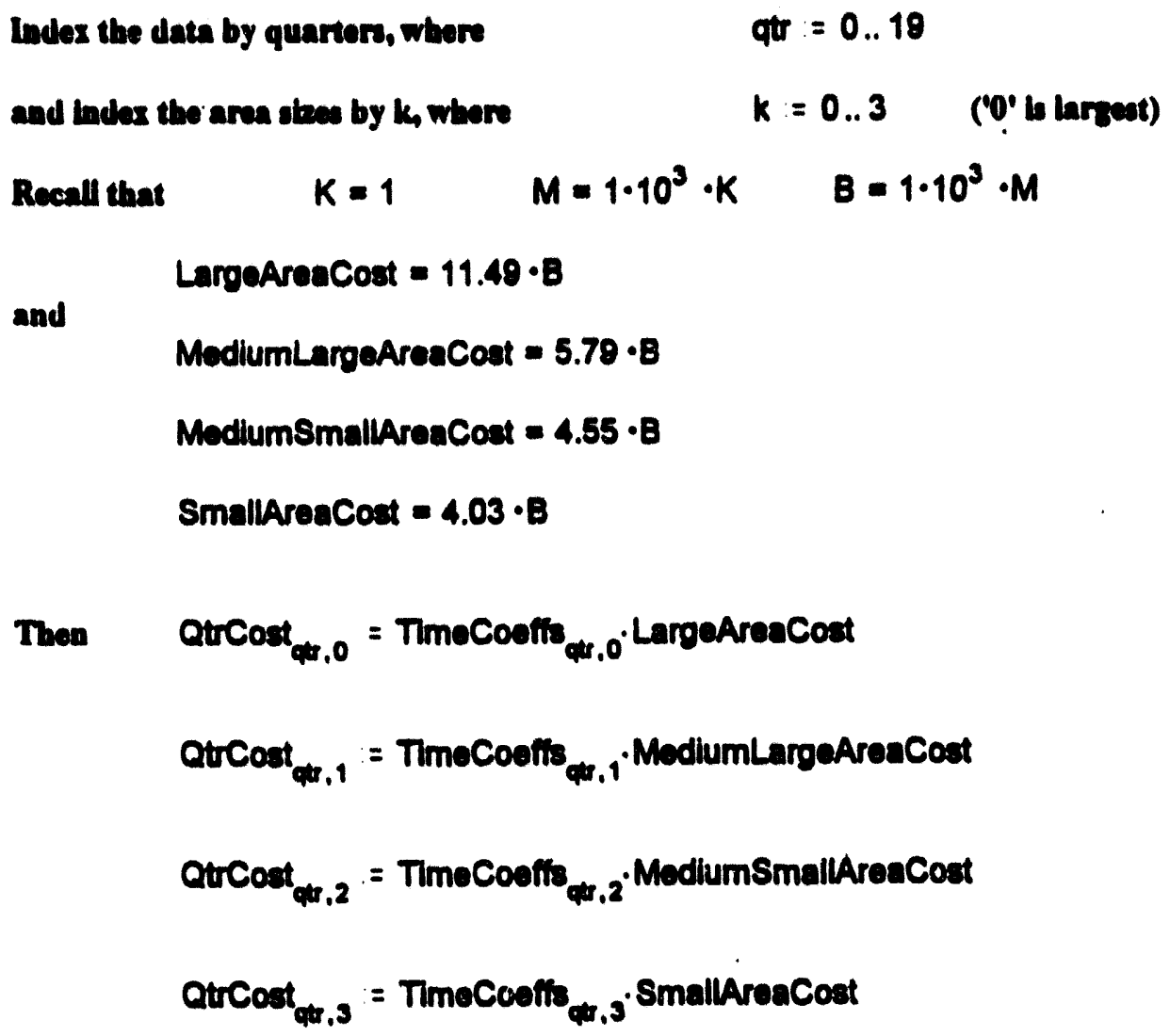

For exnmple,

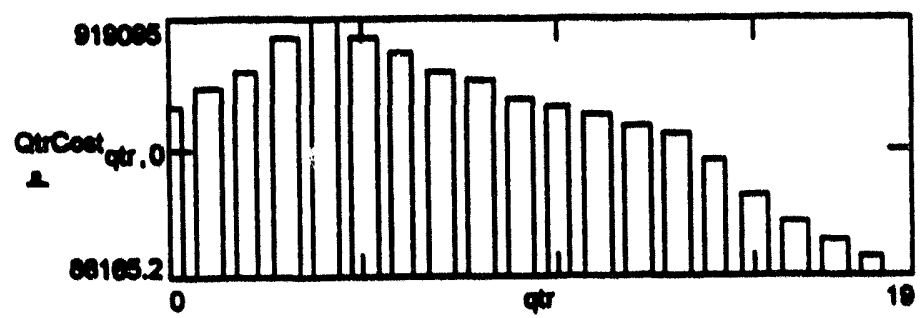

To get total implementation cost by year, we sum over the area coats and then sum the quarters in groups of four.

$$
\operatorname{QtrSum}_{\phi}:=\sum_{k} \operatorname{QtrCost}_{\phi, k}
$$




$$
\begin{aligned}
& \text { AnnualCost }_{0}:=\sum_{\text {qtr }} \text { QtrSum }_{\text {qur }} \cdot(\text { qtr }<4) \\
& \text { AnnualCost } 02.93 \cdot B \\
& \text { AnnualCost } 1:=\sum_{q t r} Q_{\text {trSum }} \cdot(q t r>3) \cdot(q t r<8) \\
& \text { AnnualCost }_{1}=6.12 \cdot \mathrm{B} \\
& \text { AnnualCost }_{2}:=\sum_{q t r} \text { QtrSum }_{\text {qur }} \cdot(q t r>7) \cdot(q t r<12) \\
& \text { AnnualCost }_{2}=8 \cdot B \\
& \text { AnnualCost } 3:=\sum_{\text {qtr }} \text { QtrSum }_{\text {qri }} \cdot(\text { qtr }>11) \cdot(\text { qtr }<16) \\
& \text { AnnualCost }_{3}=6.13 \cdot \mathrm{B} \\
& \text { AnnualCost }_{4}:=\sum_{q t} \text { QtrSum }_{\text {qtr }} \cdot(\text { qtro 15) }
\end{aligned}
$$

AnnualCost $_{4}=2.68 \cdot B$

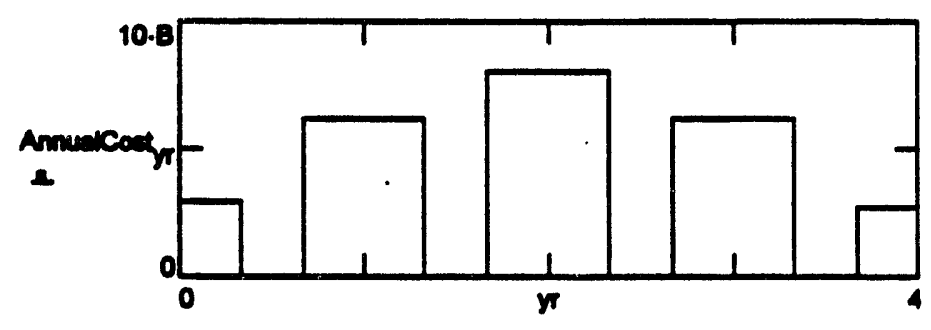

As a check,

$$
\begin{aligned}
& \text { SumOrCost : }=\sum_{y r} \text { AnnualCost }_{y r} \quad \text { SumOfCost }=25.86 \cdot B \\
& \text { GrandTotalCapital }=25.86 \cdot B
\end{aligned}
$$



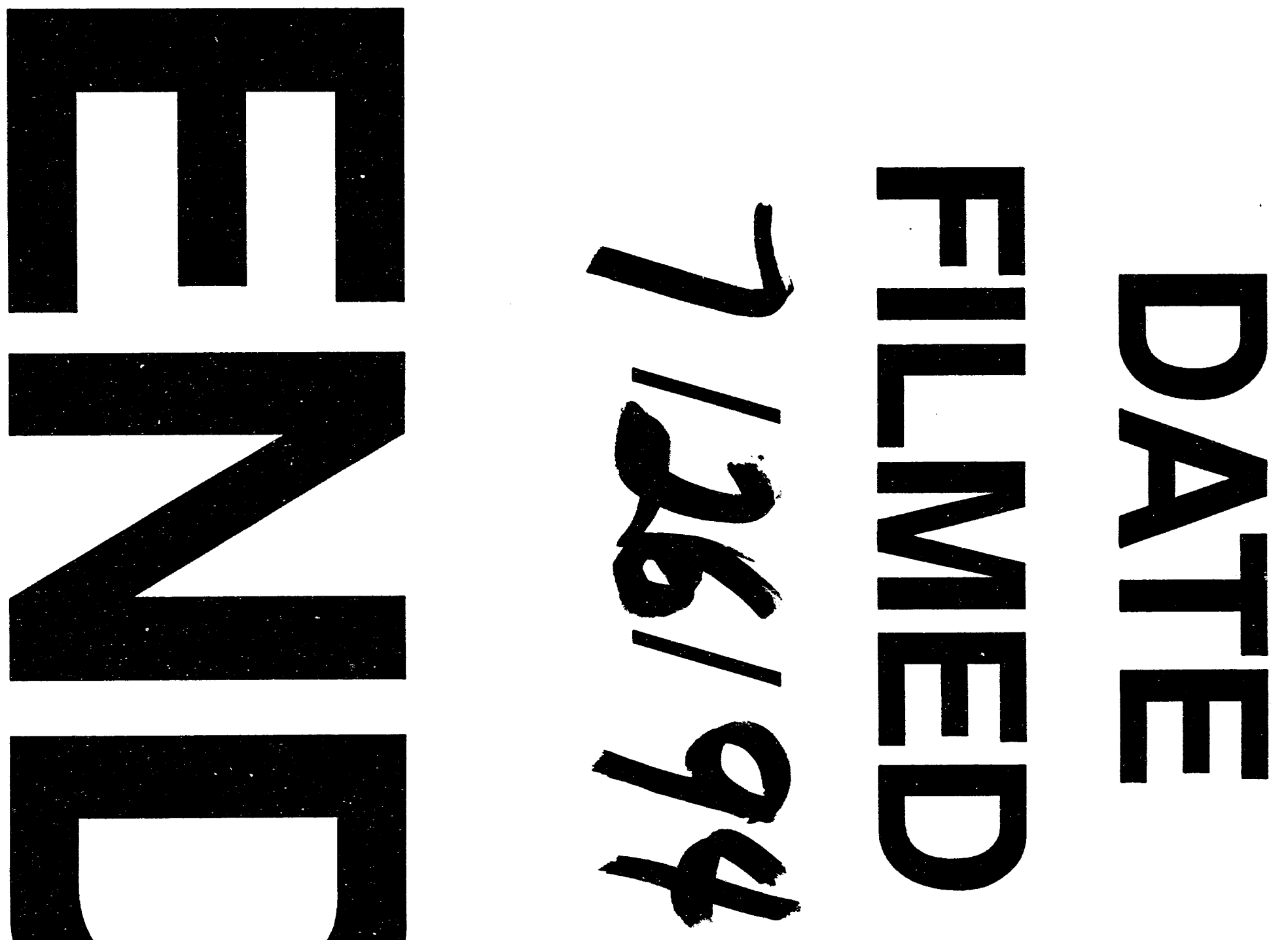


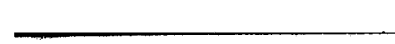

